\title{
Real photonic waveguides: guiding light through imperfections
}

\author{
Daniele Melati ${ }^{1, *}$, Andrea Melloni ${ }^{1}$ and Francesco \\ Morichetti $^{1}$ \\ ${ }^{1}$ Dipartimento di Elettronica, Informazione e Bioingegneria, Politecnico di \\ Milano, \\ via Ponzio 34/5, 20133 Milano, Italy \\ *Corresponding author: daniele.melati@polimi.it
}

Real photonic waveguides are affected by structural imperfections due to fabrication tolerances that causes scattering phenomena when the light propagates through. These effects result in extrinsic propagation losses associated with the excitation of radiation and backscattering modes. In this work, we present a comprehensive review on the extrinsic loss mechanisms occurring in optical waveguides, identifying the main origin of scattering loss and pointing out the relationships between the loss and the geometrical and physical parameters of the waveguides. Theoretical models and experimental results, supported by a statistical analysis, are presented for two widespread classes of waveguides: waveguides based on total internal reflection (TIR) affected by surface roughness, and disordered photonic crystal slab waveguides (PhCWs). In both structures extrinsic losses are strongly related to the waveguide group index, but also the mode shape and its interaction with waveguide imperfections must be considered to accurately model the scattering loss process. It is shown that as far as the group index of PhCWs is relatively low $\left(n_{g}<30\right)$, many analogies exist in the radiation and backscattering loss mechanisms with TIR waveguides; conversely, in the high $n_{g}$ regime, multiple scattering and localization effects arise in PhCWs that dramatically modify the waveguide behavior. The presented results enable the development of reliable circuit models of photonic waveguides, which can be used for a realistic performance evaluation of optical circuits. (C) 2013 Optical Society of America

OCIS codes: $130.2790,130.5296,290.0290,290.1350,290.4210$

\section{Introduction}

Optical waveguides are the pathways along which the light can be routed inside photonic chips and the basic elements to realize any photonic integrated 
circuit. As waveguides properties directly affects the circuit performance, it is of primary importance to identify the physical mechanisms impairing the light propagation and to have reliable models describing the realistic behaviour of optical waveguides.

Different strategies can be used to provide lateral confinement in dielectric waveguides, ideally inhibiting radiation to escape in the transverse plane. Conventionally, lateral confinement is achieved by means of total internal reflection (TIR), that is by making the light propagate through a high-refractive-index core material surrounded by a lower-refractive- index cladding region. Since the first concepts of dielectric optical waveguide were based on TIR [1-3], in this paper TIR waveguides will be referred to as classical waveguides. More recently TIR confinement has been exploited also in non-classical waveguides as SubWavelength Grating (SWG) structures $[4,5]$.

Alternatively, light confinement can be achieved by creating artificial band gaps to inhibit light propagation along certain directions. The most widespread example is represented by photonic crystal slab waveguides (PhCWs) [6]. In these structures, a guiding channel can be obtained by modifying [7] or removing $[8,9]$ a row of holes in the periodic hole lattice realized in a membrane. In PhCWs the light is vertically confined into the membrane by TIR, while lateral confinement is provided by the stop bands of the photonic crystal lattice [10]. Other examples of waveguides exploiting stopbands for radiation modes are multilayer hollow core waveguides, such as Bragg cladding waveguides $[11,12]$ and antiresonant reflecting optical waveguides (ARROW) [13,14], where the optical field is bounded inside a low index core by interference effects occurring in the surrounding multilayered cladding.

Theoretically, as far as the materials realizing the waveguide are transparent, all these waveguide geometries are intrinsically lossless, that is they can guide the light for an arbitrarily long distance without attenuation. However, in real life, fabrication tolerances in the technological processes introduce defects and imperfections in the waveguide geometry, thereby originating what are usually referred to as extrinsic losses. Extrinsic losses are associated with the coupling of the forward propagating guide mode(s) with radiation modes (radiation loss) as well as with the backward propagating mode(s) (backscattering), and are typically predominant compared to the loss contribution due to material absorption. In classical waveguides, extrinsic losses are mainly originated by residual surface roughness at the waveguide core boundaries, as is visible in the photo of Fig. 1(a), while in PhCWs disorder effects due to distortions of the holes shape and their random displacements with respect to the idle lattice represent an additional source of loss [see Fig. 1(b)].

In this work, we present a comprehensive review on the extrinsic loss mechanisms occurring in optical waveguides, with the aim to point out not only the main sources of loss, but also the relationships between the loss and the geometrical and physical parameters of the waveguides. Theoretical models and experimental results are presented for both classical waveguides and $\mathrm{PhCWs}$, 
(a)

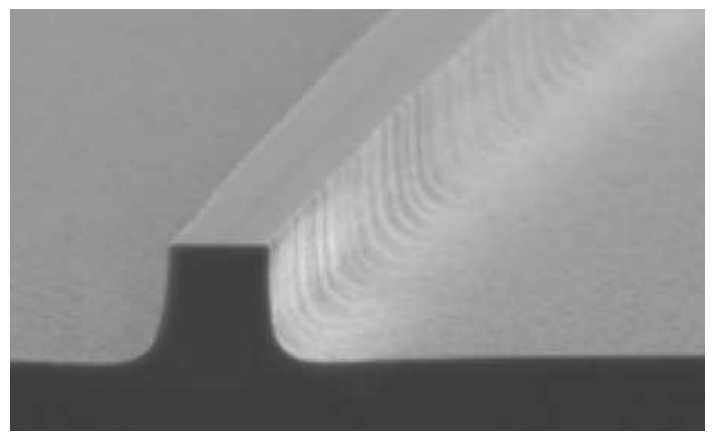

(b)

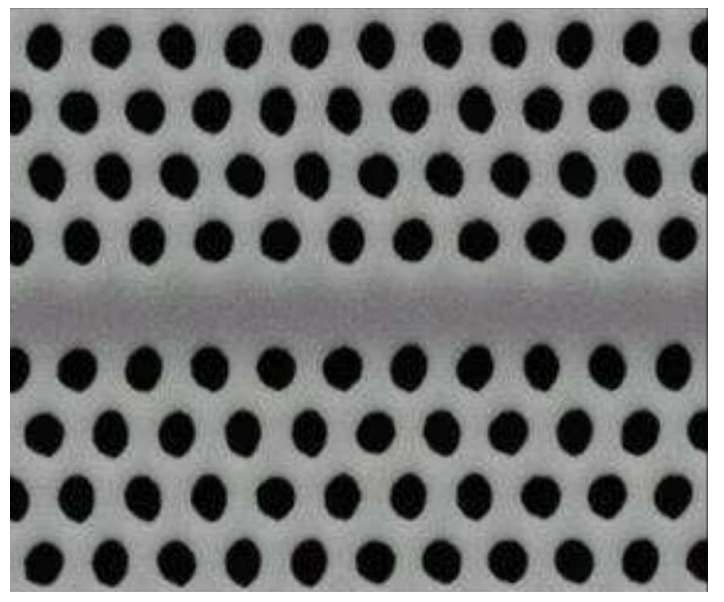

FIGURE 1. (a) SEM photograph of an uncovered classical waveguide. Sidewall roughness is clearly visible at the sidewall of the core region (adapted from [15]). (b) Top-view SEM photograph of a PhCWs showing typical disorder effects produced by fabrication tolerances. In this device the degree of disorder was intentionally enhanced to investigate the effect on the light propagation (adapted from [16]).

demonstrating that many analogies can be found in both the radiation loss and the backscattering suffered by these two different waveguiding concepts, as far as the group index $n_{g}$ of PhCWs remain relatively low $\left(n_{g}<30\right)$. Conversely, in the high $n_{g}$ regime, multiple scattering and localization effects arise in PhCWs, which dramatically change its behavior. These phenomena have no analogies in classical waveguides.

Sections 2 to 4 deal with the analysis of classical waveguides affected by sidewall roughness. More in detail, in Sec. 2 the origins and characteristics of roughness are discussed and theoretical models are presented taking into account the statistical properties of the waveguide roughness. Section 3 provides a theoretical and experimental analysis of roughness-induced radiation loss in classical waveguides. The most established theoretical models reported in the literature are presented and are compared to a model, referred to as $n_{w}$ model, which is based on the sensitivity of the waveguide effective index $n_{\text {eff }}$ to per- 
turbations of the waveguide width $w$. In Sec. 4 , the $n_{w}$ model is applied to the study of roughness-induced backscattering in classical waveguides and results are compared to other models reported in the literature. Experimental results that point out the role of polarization state, index contrast and waveguide shape in the backscattering process are also provided.

Disordered PhCWs are the subject of Secs. 5 to 7 . A theoretical model describing disordered $\mathrm{PhCWs}$ is introduced in Sec. 5, where a classification of the main statistical disorder parameters to be considered for a realistic modelling of $\mathrm{PhCWs}$ is provided. Radiation and backscattering losses in $\mathrm{PhCW}$ are theoretically and experimentally discussed in Sec. 6, with the help of a generic model that can be applied to arbitrary $\mathrm{PhCW}$ geometries. The role of the group velocity, Bloch mode shape and correlation length is discussed, to point out the main analogies and difference with classical waveguides. Section 7 specifically focuses on multiple scattering effects arising in $\mathrm{PhCW}$ s in the high group index regime $\left(n_{g}>30\right)$, such as the breakdown of the Beer-Lamber law and the existence of localized states, which have no counterpart in classical waveguides.

Due to the inherent randomness of roughness and disorder effects, a statistical analysis of both classical and PhCWs is provided in Secs. 8 to 10. Based on the statistical properties of the backscattering process (Sec. 8), a realistic and accurate circuit model of classical waveguides affected by sidewall roughness is developed in Sec. 9. Then, the statistics of the light intensity transmitted and backscattered in PhCWs is experimentally derived in Sec. 10, pointing out the similarity between the behaviour of $\mathrm{PhCWs}$, operating in the low $n_{g}$ regime, and classical waveguides.

The impact of the backscattering effects on the behaviour of optical devices, such as optical resonators and Bragg gratings, is discussed in Sec. 11. In Sec. 12 is it shown that disorder in coupled resonator structures made of classical waveguides can produce effects that are analogous to those arising in disordered PhCWs operating in the high-group-index regime, leading to multiple scattering and localization phenomena. The final Section 13 summarizes the main results of the paper, highlighting the difference and the analogies between the effects of fabrication tolerances on the extrinsic loss of classical waveguides and $\mathrm{PhCWs}$.

\section{Origin and Characteristics of Waveguides Sidewall Roughness}

Waveguide sidewall roughness is an unavoidable physical imperfection that affects all kinds of integrated optical components. It is a surface perturbation that produce a local variation of the waveguide width and consequently induces a local spatial fluctuation of the effective refractive index. Roughness is generated during the realization processes of the planar optical circuits and in particular by the etching of the core layer. The quality of the masks used during the photolithographic process is fundamental to obtain smooth sidewalls because the etching tends to reproduce the mask profile on the underlying substrate with great detail $[17,18]$. The resolution of photoresist, the type of lithography - beam 
or optical-, the number of etching steps required for the waveguide definition and the waveguide materials are other important factors that impact on the roughness generated along the sidewalls [19].

Figure 2(a) shows a sketch of the geometry and the reference frame used for the analysis. Due to its origin, sidewall roughness has very different characteristics if observed in the vertical direction (y direction) or in the propagation direction (z). In most of cases the roughness can be assumed as vertical streaks on the sidewall. In practice, in some deeply etched waveguides it has been observed also a weak dependence of the roughness on $y$ [15]. Under particular circumstances this dependence induces the coupling between the TE and TM modes, as explained in [20], but does not change substantially the scattering behaviour. In this paper a columnar roughness is assumed, reducing the problem to a $2 \mathrm{D}$ one. The validity of this assumption is evident from Fig. 2(b) and (c), as discussed in the following.

\subsection{Mathematical Description of Roughness}

The roughness can be defined by means of a zero-mean random function $f(z)$ which describes the local deviation of the waveguide width from the ideal location (see Fig. 2(a), right). The transverse refractive index profile at location $\bar{y}$ (with $0<\bar{y}<h$ ) is then given by

$$
n(x, \bar{y}, z)= \begin{cases}n_{1}, & \text { if }|x|<w / 2+f(z) \\ n_{2}, & \text { if }|x|>w / 2+f(z)\end{cases}
$$

where $n_{1}$ and $n_{2}$ are the refractive indexes of core and cladding, respectively, $w$ the waveguide width and $h$ the thickness.

The roughness function $f(z)$ and its statistical properties are commonly described through the ensemble autocorrelation function

$$
R\left(u_{z}\right)=\left\langle f(z) f\left(z-u_{z}\right)\right\rangle
$$

being $u_{z}$ the displacement in the $\mathrm{z}$ direction. $R\left(u_{z}\right)$ measures the correlation between two points on the sidewall separated by a distance $u_{z}$. The correlation length $L_{c}$ of the process is defined as the half width of $R\left(u_{z}\right)$ at $1 / e$ value from the maximum. Assuming the roughness being a wide-sense-stationary (WSS) random process and a correlation length $L_{c}$ much smaller than the length of the whole waveguide $L_{w}$ (approximately uncorrelated process, as actually happens in most practical cases), the process can be considered approximately ergodic and the ensemble average in Eq. (2) substituted with an average on the length of the waveguide [22]

$$
R\left(u_{z}\right)=\lim _{L_{w} \rightarrow+\infty} \frac{1}{L_{w}} \int_{-L_{w} / 2}^{L_{w} / 2} f(z) f\left(z+u_{z}\right) d z
$$

As consequence the WSS assumption, $R\left(u_{z}\right)$ results to be a monotonically decreasing function of width equal to $L_{c}$. For a completely uncorrelated process 

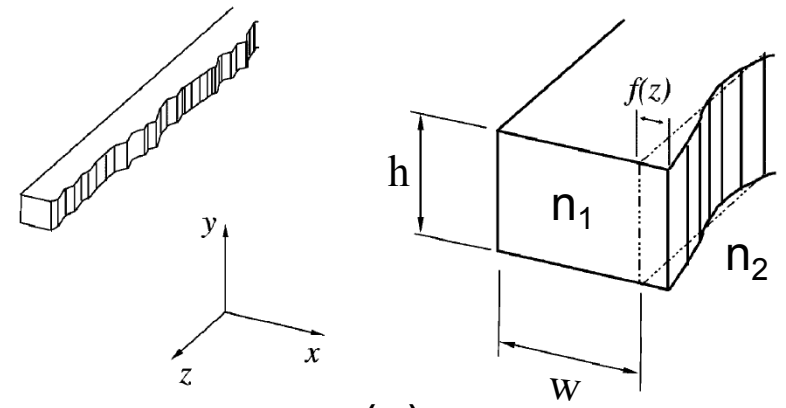

(a)

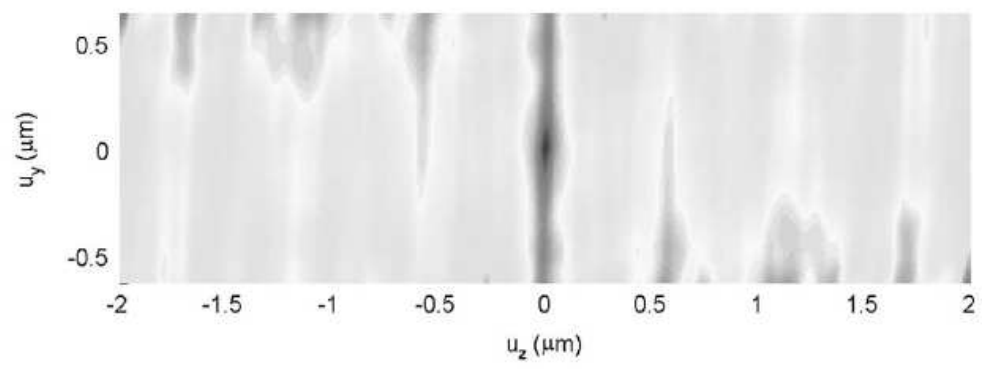

(b)

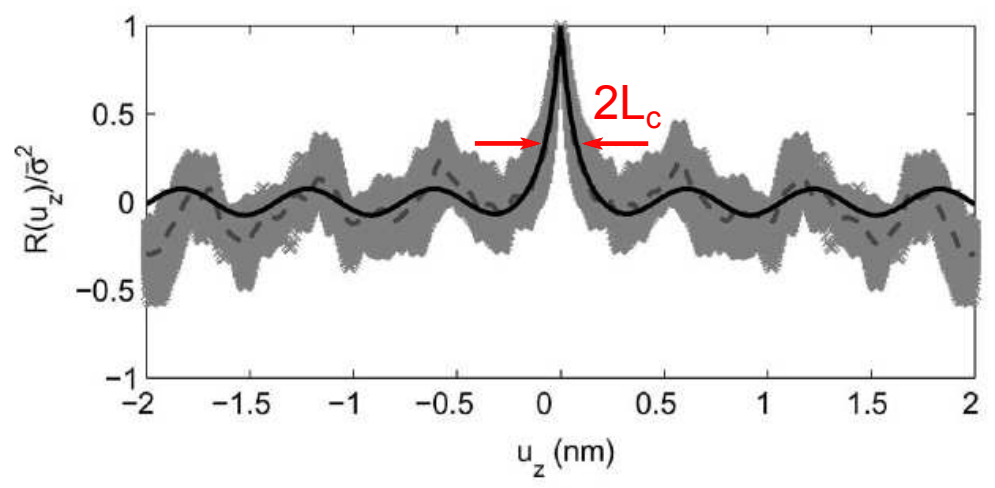

(c)

FIGURE 2. (a) Sketch of a waveguide with sidewall roughness and reference frame. The roughness is assumed $y$ independent. The random function $f(z)$ describes the corrugation profile. (b) Measured autocorrelation function of the sidewall roughness for a pedestal waveguide as function of the displacement $u_{z}$ and $u_{y}$ in the $\mathrm{z}$ direction (propagation direction) and $\mathrm{y}$ direction (vertical direction), respectively. The invariance in the vertical direction is clearly visible. (c) Autocorrelation functions along direction $\mathrm{z}$ for different vertical positions over the wall (shaded gray), average value of the set (dashed line) and fit with the exponential model (solid line). (adapted from [21]) 
(white noise) $L_{c}=0$ while a deterministic behaviour would be characterized by $L_{c} \rightarrow+\infty[17]$.

The WSS assumption allows also to apply the Wiener-Khintchine's theorem which states that $R\left(u_{z}\right)$ has a spectral decomposition $S(\theta)$ equal to the power spectrum of $f(z)[22]$

$$
S(\theta)=\frac{1}{2 \pi} \int_{-\infty}^{+\infty} R\left(u_{z}\right) e^{-i \theta u_{z}} d u_{z}
$$

where $\theta$ refers to spatial frequency of the sidewall roughness.

\subsection{Statistical Properties}

Two different models have been proposed in the past to describe the statistical properties of the sidewall roughness [23-25]. The first one exploits a Gaussian correlation model in the form

$$
R\left(u_{z}\right)=\sigma^{2} \exp \left(-\frac{u_{z}^{2}}{L_{c}^{2}}\right)
$$

being $\sigma$ the root-mean-square roughness, $L_{c}$ the correlation length of the function $f(z)$ and $u_{z}$ the displacement along the direction $z$. The second model relies on an exponential autocorrelation function

$$
R\left(u_{z}\right)=\sigma^{2} \exp \left(-\frac{u_{z}}{L_{c}}\right) .
$$

Numerous experimental investigations [17,26], based on Atomic Force Microscope (AFM) measurements of the roughness profiles, revealed that its autocorrelation function can be well approximated by Eq. (6) or equivalently

$$
S(\theta)=\frac{\sigma^{2}}{\pi} \frac{L_{c}}{1+L_{c}^{2} \sigma^{2}}
$$

This model is nowadays commonly accepted for lithographically defined waveguides [21,27-31].

As an example, Fig. 2(b) shows a bi-dimensional autocorrelation function $R\left(u_{z}, u_{y}\right)$ of the waveguide roughness in the core region for an InGaAsP pedestal waveguide. AFM data have been normalized in order to produce a zero-mean function $f(y, z)$ and compute $R\left(u_{z}, u_{y}\right)$ [21]. As expected, the roughness is strongly correlated in the vertical direction $\left(u_{y}\right)$ because of the anisotropic etching process and, in this particular case, the correlation length in this direction has been found to be larger than the core width, experimentally confirming the assumption of y-invariance of the roughness. The mono-dimensional autocorrelation function necessary to describe the statistical properties of the roughness is plotted in Fig.2(c) for many different $\bar{y}$ positions on the waveguide sidewall (shaded grey) along with the average value (dashed line). The data can be reasonably fitted with Eq. (6) with $L_{c}=(56 \pm 14) \mathrm{nm}$ and $\sigma=(5 \pm 1) \mathrm{nm}$ (black solid line). 
Typical values of standard deviation and correlation length of the roughness for different types of waveguides are reported in Tab. 1, ordered by decreasing refractive index contrast $\Delta n=\left(n_{1}-n_{2}\right) / n_{2}$. As can be seen, the correlation length of the roughness is generally in the order of tens on nanometers and hence the assumption $L_{c}<<L$ holds well and the stocastic process can be referred as approximately white noise $\left(L_{c} \simeq 0\right)$.

TABLE 1. Tipical values of refractive index contrast, root-mean-square roughness and correlation length for several production technologies.

\begin{tabular}{|c|c|c|c|c|}
\hline Technology & $\Delta \mathbf{n} \%$ & $\sigma[\mathrm{nm}]$ & $\mathbf{L}_{c}[\mathbf{n m}]$ & ref \\
\hline SOI & 140.0 & $<2$ & 50 & $\overline{[32-34]}$ \\
\hline $\mathrm{Si}_{3} \mathrm{~N}_{4}$ & 37.0 & $3.5 \div 14$ & 50 & {$[31,35]$} \\
\hline $\mathrm{SiON}$ & $1.0 \div 4.5$ & $<3^{*}$ & - & {$[36]$} \\
\hline InP/InGaAsP & $3.0 \div 5.0$ & $<5$ & 50 & {$[37,38]$} \\
\hline Silica & $<1.0$ & $<4^{*}$ & - & {$[39]$} \\
\hline
\end{tabular}

$\left(^{*}\right)$ measured on etched planar films

\section{Waveguide radiation losses}

Several contributions can be identified as responsible of the attenuation experienced by the mode propagating in an optical waveguide. Assuming a weak attenuation, the power insertion loss of a waveguide of length $L_{w}$ can be written as

$$
I L=e^{\alpha L_{w}}=e^{\left(\alpha_{a}+\alpha_{r}+\alpha_{b}\right) L_{w}}
$$

where $\alpha_{a}$ takes into account the pure material absorption while $\alpha_{b}$ and $\alpha_{r}$ describes the fraction of the light coupled to a counter-propagating mode (described in the next section) and to radiative modes, respectively. As $\alpha_{b}$ and $\alpha_{r}$ are associated with sidewall roughness, they represent the extrinsic loss contribution. The coupling effects involved in the latter contributions are related to the interaction between the propagating mode and the sidewall surface roughness. This interaction couples the incoming propagating guided mode to all the other guided modes, forward and backward, and to the continuum spectrum of radiative modes $[40,41]$. Since $\alpha_{b}$ is generally small compared to the other contributions, the previous equation can be approximated as

$$
I L \simeq e^{\left(\alpha_{a}+\alpha_{r}\right) L_{w}} \cdot\left(1-\alpha_{b} L_{w}\right)
$$

where the loss coefficient $\alpha_{b}$ corresponds to the parameter $r_{b}$ commonly used to describe the backscattered power per unit length. 
Radiative losses and their connection to the waveguide geometry have been widely investigated in the last decades $[33,42]$ in particular for high-indexcontrast technologies such as SOI, which can be very sensitive to sidewall imperfections. Large efforts have also been devoted to the technological processes to reduce this source of loss, acting in particular to smooth the sidewalls through repeated oxidations and advanced lithographic techniques $[32,43]$ and silicon wire waveguides with overall losses smaller than $1 \mathrm{~dB} / \mathrm{cm}$ have been reported [19,44].

Several models have been investigated to study and predict the power loss generated by random imperfection of waveguide sidewalls. As already mentioned, early works by Marcuse $[40,41]$ modelled the radiative losses as the coupling between the propagating mode and radiative modes involving an elaborate integral over the modes of the unguided continuum. In these papers, results were applied to 2-D slabs and circular waveguides. The former have been analyzed also by Payne and Lacey who developed a technique to compute the radiation losses treating the waveguide as a radiating antenna [24,25]. This method is detailed presented in the following section along with its (approximate) extension to the three dimensional case. A different 2-D approach applied to thin films problem has been proposed by Tien exploiting simple specular reflection laws [45].

More accurate results are expected from fully-three-dimensional treatments of the scattering phenomena which could take into account the extra degree of freedom of the 3-D radiation modes and giving an accurate description of the impact of the waveguide cross section on the radiation efficiency. A common approach to address this problem is the volume current method. This is a perturbation technique where the radiation field is represented as the far field generated by an equivalent volume polarization current density and the losses are calculated as the integral over the radiation pattern. The method has been applied to both classical waveguides (low- and high-contrast) and photonic crystals [31,46-48]. Extensions of the Marcuse's coupled-mode theory to a 3-D problem have been proposed as well for both low-contrast [49] and high-contrast [21] waveguides. Other approaches rely on the boundary-condition method [50] or generalization of the scattering matrix formalism to describe a rough waveguide as a cascade of abrupt discontinuities [51].

Among these, the Payne and Lacey model for a 2-D slab waveguide is widely exploited in literature for the estimation of the radiation losses. Details of this technique are discussed in Sec.3.1 where a comparison with a model based on the sensitivity of the waveguide effective index $n_{e f f}$ to perturbations of the waveguide width $w$ is proposed.

\subsection{A new insight into the Payne-Lacey model}

In the Payne and Lacey model [24,25], the geometry of the problem consists of a symmetric slab waveguide of width $w$ and with core and cladding refractive indexes $n_{1}$ and $n_{2}$, respectively. The random waveguide sidewall perturbation is described by the root-mean-square roughness $\sigma$ and correlation length $L_{c}$ as presented in the previous section. 
The method allows the computation of the far field radiated by the waveguide as consequence of the surface roughness. The methodology is based on the method of the equivalent currents and assumes the waveguide to act as a radiating antenna, with the roughness acting as an equivalent current source. Applying conventional radiation methods, it is possible to derive from the radiation pattern an expression for the exponential coefficient $\alpha_{r}$,

$$
\alpha_{r}=\frac{\sigma^{2}}{\sqrt{2} k_{0}(w / 2)^{4} n_{1}} g f
$$

where $k_{0}$ is the wave number. The functions $g$ and $f$ (refer to $[24,25]$ for the complete expressions) are the most remarkable aspect of this model because allow to understand how different waveguide parameters contribute to radiative losses. The function $g$ is determined only by the waveguide geometry (slab width); $f$ is related to the correlation length of the sidewall perturbation and takes into account the interaction between the propagating mode and the sidewall perturbation. Both functions depend also on the refractive index distribution of the waveguide and the mode effective refractive index $\left(n_{e f f}\right)$ through the propagation constant $\beta$. Finally, note that $\alpha_{r}$ is predicted proportional to the square of the roughness standard deviation $\sigma$.

Intriguingly, we found that the attenuation predicted by Eq. (10) as function of the slab width $w$ perfectly matches the derivative of the effective refractive index with respect to the slab width

$$
\alpha_{r}=A \frac{\partial n_{e f f}}{\partial w}
$$

through a proportionality factor $A$ independent on the slab width and taking into account only the roughness standard deviation and correlation length. The model based on Eq.(11) is named here $n_{w}$ model because it is inherently based on the "sensitivity" of the mode effective index to the width variations produced by the sidewall roughness and consequently to the amount of power coupled out to the radiative modes.

Figure 3 shows a comparison between the two presented models for a variety of slab widths, index contrast and propagating modes once the roughness parameters have been fixed to $\sigma=2 \mathrm{~nm}$ and $L_{c}=50 \mathrm{~nm}$. In Fig. 3(a) the predicted loss coefficients as function of the waveguide width $w$ are shown for the TE fundamental mode of three different slabs. The waveguides differ in the index contrast between core and cladding $\Delta n=\left(n_{1}-n_{2}\right) / n_{2}$, which changes from $\Delta n=3 \%$ (e.g. glass waveguides) to $\Delta n=30 \%$ (e.g. SiN waveguides) and $\Delta n=90 \%$ (high index contrast technology). In all the three cases the results are perfectly superposed.

In Fig. 3(b) the loss coefficient $\alpha_{r}$ has been calculated only for the waveguide $\Delta n=30 \%$ with the same roughness parameters of the previous example and for the first three TE propagating modes. For the fundamental, first and second higher order modes the agreement is excellent, apart a slight difference near the modes' cut-offs. The same results have been obtained with TM modes. 


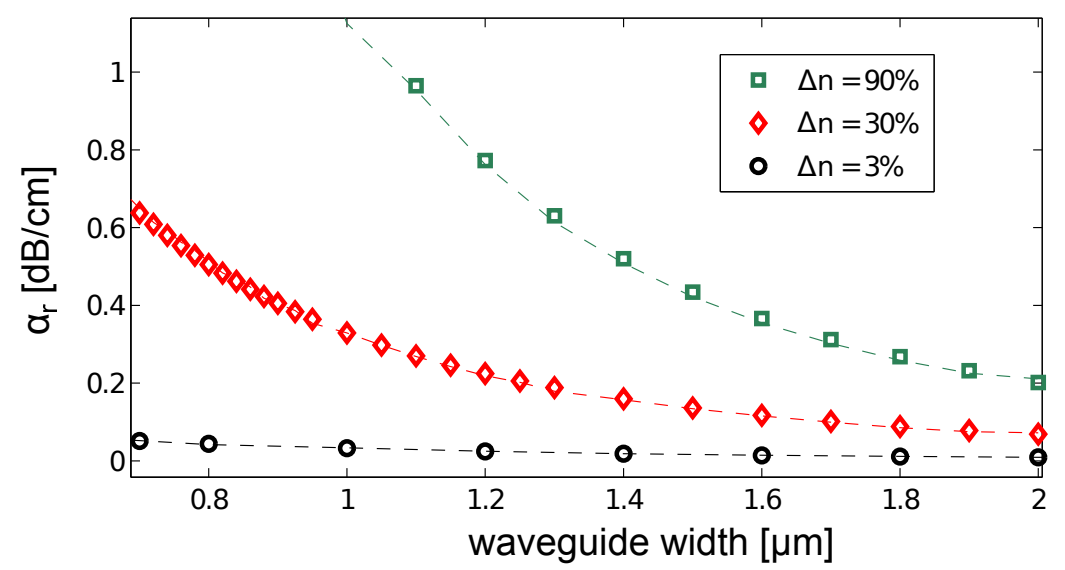

(a)

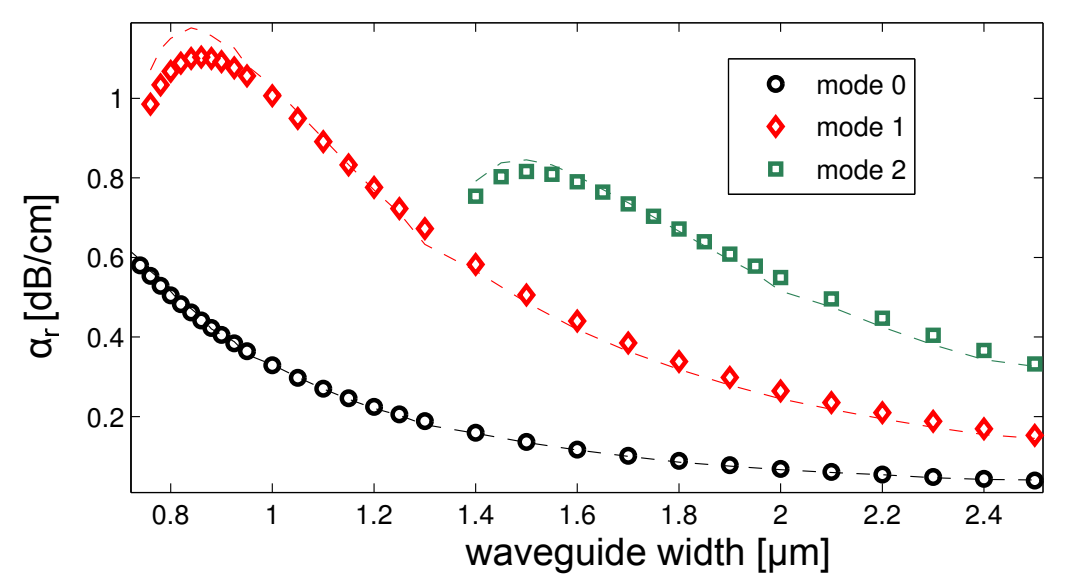

(b)

FIGURE 3. Comparison between the losses predicted by the Payne-Lacey model (dots) and the $n_{w}$ model (dashed lines) for (a) slabs with different index contrast and fixed roughness parameters $\left(\sigma=2 \mathrm{~nm}, L_{c}=50 \mathrm{~nm}\right)$; (b) different modes of the slab with $\Delta n=30 \%\left(\sigma=2 \mathrm{~nm}, L_{c}=50 \mathrm{~nm}\right)$.

\section{2. $3 D$ waveguides and experimental results}

The Payne-Lacey model was originally developed only for 2-D slab waveguides but more recently has been applied also to $3-\mathrm{D}$ structures. The dimensionality of the problem can be reduced applying the effective index method $[28,30]$ to obtain a 2-D slab equivalent to the original waveguide and approximating the 3 -D radiative modes with $2-\mathrm{D}$ planar radiative modes. Both cited studies are related to buried-like SOI waveguides, which can be well approximated by 2-D slabs [21], ensuring a good agreement between the numerical model and the experimental results. 
The most interesting extension of the Payne-Lacey model to a 3-D waveguide has been proposed by Yap et al. [52] who developed a correction of the model valid also for rib waveguides (called ridge in [52]). The problem with rib waveguides is that the Payne-Lacey model tends to overestimate the scattering losses from the partially etched sidewalls. With simple electromagnetic and variational considerations, the authors suggest a relation between the variation of the mode effective index induced by the waveguide width perturbation and the overlapping integral of the electric field with the refractive index perturbation at the location of the scattering defect (which generate the coupling with the radiative modes).

Assuming a rib waveguide with etch depth $h$ and width $w$, the modified PayneLacey model proposed by Yap et al. can be written in the form

$$
\begin{gathered}
\alpha_{r}=\frac{\sigma^{2}}{\sqrt{2} k_{0}(w / 2)^{4} n_{e f f}} g f s, \\
s=\frac{\partial n_{e f f r} / \partial w}{\partial n_{e f f c} / \partial w}
\end{gathered}
$$

where the mode effective index $n_{\text {eff }}$ substitutes the core refractive index of Eq. (10) (as effect of the application of the effective index method to reduce the 3D problem to 2D) and a dimensionless scaling factor $s$ has been added. This scaling factor represents the ratio between the differential change of the effective index for a rib waveguide $n_{\text {effr }}$ and for a channel waveguide $n_{\text {effc }}$ when the same width variation is applied. When the etch depth increases, the rib waveguide tends to the channel waveguide and $s$ approaches unity. On the other hand, when the rib waveguide becomes shallower, $s$ tends to zero and the sidewall scattering losses vanishes.

An example of application of Eq. (12) is shown in Fig. 4(a) [52] which presents the TE mode propagation losses for a SOI rib waveguide as function of the waveguide width. The data points represent the experimental results obtained for the same waveguide geometry realized with three different technological processes. Each process generates a sidewall roughness with different characteristics in terms of standard deviation $\sigma$ and correlation length $L_{c}$ and consequently a different dependence of the radiative losses on the waveguide width. The experimental data are in good agreement with the theoretical losses predicted by the modified Payne-Lacey model (Eq. (12), solid line) for a given combination of $\sigma$ and $L_{c}$.

The scaling factor $s$ given by Eq. (13) confirms the match between the Eqs. (10) and (11) and suggests that the proportionality between $\alpha_{r}$ and the derivative of $n_{\text {eff }}$ holds also in the 3 -D case. By using Marcatili approximation for rectangular waveguides [53], Eq. (11) can be generalized to 3-D structures as

$$
\alpha_{r}=A \frac{\partial n_{e f f}}{\partial \mathbf{n}} \simeq A\left[\frac{\partial n_{e f f}}{\partial w}+\frac{\partial n_{e f f}}{\partial h}\right]
$$

being $\mathbf{n}$ the normal versor to the waveguide boundaries. The factor $A$ incorporates in this case also the scaling factor $s$ of Eq. (12) along with all the 


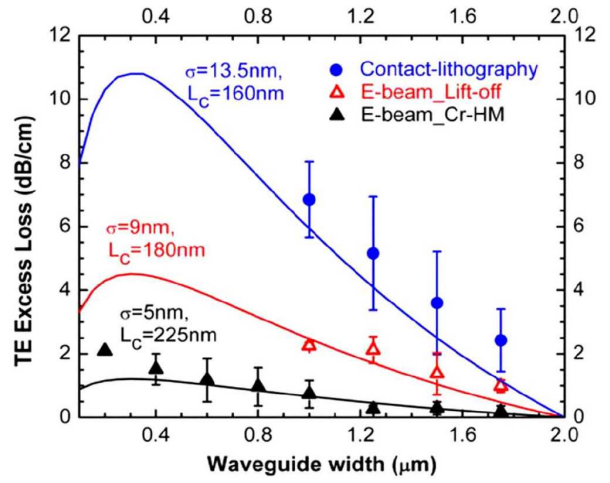

(a)

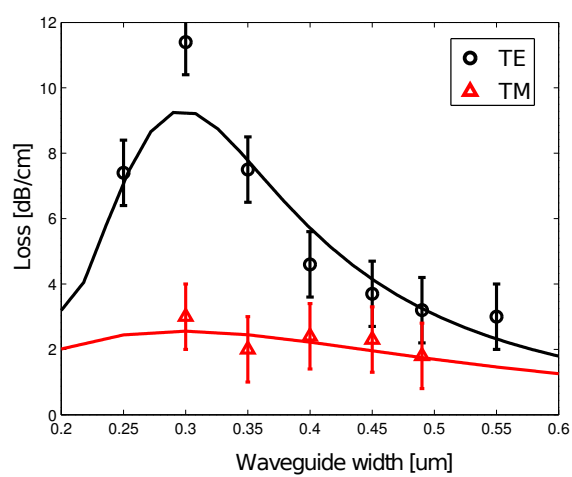

(b)

FIGURE 4. Measurements (marks) and model predictions (solid lines) of the propagation losses versus width of different types of waveguides: (a) TE mode of a SOI rib waveguide fabricated by three different processes and application of the Payne-Lacey model with a scaling factor (adapted from [52]); (b) TE and TM modes of a channel SOI waveguide and application of the $n_{w}$ model (experimental data are taken from [20]).

parameters which do not depend on the waveguide width. This equation allows to take into account also the contribution to the radiative loss generated by the roughness of the top/bottom surfaces parallel to the waveguide plane. However, this contribution is typically negligible compared to sidewall roughness and the model (14) reduces simply to Eq. (11).

Figure 4(b) shows the experimental propagation loss of a SOI channel waveguide with $h=220 \mathrm{~nm}$ for both TE (black dots) and TM (red triangles) fundamental modes. The results have been fitted with Eq. (14) where, as in the 2-D example of the previous paragraph, the only free parameter is represented by the width-independent factor $A$. As in the case of the modified Payne-Lacey model, a good match can be observed between the experimental data and the fitting model for both modes. This is true even in the region around $w=0.3 \mu \mathrm{m}$ where the model predicts a strong enhancement of the propagation losses.

\subsection{Final remarks about radiative losses}

Some concluding considerations are worth to be done about the presented models for the radiative losses induced by the waveguide sidewall roughness. The Payne-Lacey model, applied to both a 2-D slab and a 3-D waveguide through the effective index method and corrected with the scaling factor $s$ if needed, can be used to predict the radiative loss coefficient $\alpha_{r}$ as function of the waveguide width once the information about the waveguide geometry and sidewall roughness has been provided. Discussions and examples of the previous paragraphs show how, in both 2-D and 3-D cases, this model basically represents the derivative of the mode effective index with respect to the waveguide width, a part from 
a constant scaling factor $\left(n_{w}\right.$ model $)$. This dependence can be demonstrated rigorously as follow.

Let's consider a symmetric slab with core index $n_{1}$, cladding index $n_{2}$ and thickness $w$. The normalized frequency can be defined as

$$
V=\frac{\omega}{c} w \sqrt{n_{1}^{2}-n_{2}^{2}}
$$

where $\omega$ is the angular frequency and $c$ the speed of light. Taking the derivative of $\mathrm{V}$ with respect to $\omega$ and $w$ results

$$
\omega \frac{\partial n_{e f f}}{\partial \omega}=w \frac{\partial n_{e f f}}{\partial w} .
$$

Combining Eq. (15) with the well-known definition of the group effective index

$$
n_{g}=n_{e f f}+\omega \frac{\partial n_{e f f}}{\partial \omega}
$$

and assuming that the core and cladding effective indexes are not frequency dependent (in which case an other waveguide-independent term must be added) the following result is found,

$$
w \frac{\partial n_{e f f}}{\partial w}=n_{g}-n_{e f f},
$$

relating the $n_{w}$ model to the difference $\left(n_{g}-n_{e f f}\right)$. This is basically another way to calculate the group index.

The dependence of the waveguide losses on the difference $\left(n_{g}-n_{\text {eff }}\right)$ is rigorous and it is related to the fact that a change in the waveguide width modifies both the group index and the mode field distribution and hence the interaction with sidewall roughness. More precisely, the difference between group and phase effective indexes is related to the relative strength of the longitudinal component of the field with respect to the transversal component. This can be shown introducing the time-averaged power $\mathrm{P}$ transported by the waveguide

$$
P=\int_{-\infty}^{+\infty} d x d y S_{z}=\int_{-\infty}^{+\infty} d x d y\left[\mathbf{E} \times \mathbf{E}^{*}+\mathbf{E}^{*} \times \mathbf{H}\right]_{z}
$$

being $S_{z}$ is the component of the Poynting vector in the propagation direction, which depends only on the trasversal component of the field. The integration is done on the whole cross-section of the waveguide. With a variational approach and simple manipulations [54] (and assuming the absence of material dispersion) the following relation is demonstrated

$$
\left(n_{g}-n_{e f f}\right)=\frac{2 c}{P} \int_{-\infty}^{+\infty} d x d y\left[\mathbf{E}_{z} \cdot \mathbf{E}_{z}^{*}+\mathbf{H}_{z} \cdot \mathbf{H}_{z}^{*}\right]
$$

where the subscripts $z$ refers to the field components in the propagation direction and $c$ is the speed of light. Eq. (19) states that the difference between $n_{g}$ and 
$n_{\text {eff }}$ goes to zeros when the longitudinal component of the propagating mode vanishes, for example when the waveguide is strongly multimode [54]. In this regime the sensitivity of the field to the sidewall roughness vanishes as well (since $\partial n_{e f f}(\partial w \rightarrow 0)$ and radiative losses become negligible.

\section{Waveguide backscatter}

As mentioned in the previous section, a second relevant effect originates from the interaction of the field with the sidewall imperfections. As roughness can couple power between the guided modes and the radiative modes, in the same way it can act as a coupling element between guided modes propagating in opposite directions [55]. Backscattering can provoke serious degradation of the optical system performances, originating a variety of impairments such as spurious responses, intersymbol interference, transfer function distortion, cross-talk, return loss degradation, and lasers diodes instability [20]. For this reason, in the rest of the paper the backscattered signal will be clearly distinguished from the radiative losses. Although it contributes to the total losses experienced by the forward-propagating mode, it can be much more disturbing and less tolerable for the system than a simple power loss.

Despite these potentially strong adverse effects, the problem of backscattering has not received as much attention in the literature as losses. In the following of the section two different proposed models to estimate the backscattered signal for a waveguide with given roughness parameters are presented. As done for the losses, both models are compared to find the dependence of the backscattering on the waveguide width and it is shown how the power backscatter coefficient $r_{b}$ (like the radiative losses $\alpha_{r}$ ) is related to the derivative $\partial n_{e f f} / \partial w$.

\subsection{Models for the roughness-induced backscattering}

A model to evaluate the backscattered signal generated by the sidewall roughness has been proposed by Ladouceur and Poladian [55] for planar slab waveguides. The geometry of the problem is the same used in Sec. 3.1: a slab of thickness $w$, core and cladding refractive indexes $n_{1}$ and $n_{2}$, respectively, root-mean-square roughness $\sigma$ and a correlation length $L_{c}$. As in the previous cases, a roughness with an exponential correlation function is considered.

Ladouceur and Poladian solve the problem in term of a system of two coupled equations that describe the power exchange between the propagating and counter-propagating modes. This description is generally valid for any type of waveguide. Assuming a slab of length $L_{w}>>L_{c}$ and a small perturbation of the sidewalls (with uncorrelated perturbations on the two sidewalls), it is possible to analytically determine the coupling coefficient of the system and compute the distributed power backscattering coefficient as

$$
r_{b}=\left[\frac{U^{2} W}{2(w / 2)^{3} \beta(1+W)}\right] \frac{\sigma^{2} L_{c}}{\pi} \frac{1}{1+4 \beta^{2} L_{c}^{2}},
$$




$$
U=\frac{w}{2}\left(k_{0}^{2} n_{1}^{2}-\beta^{2}\right)^{1 / 2}, \quad W=\frac{w}{2}\left(\beta^{2}-k_{0}^{2} n_{2}^{2}\right)^{1 / 2}
$$

where $\beta$ is the mode propagation constant and $k_{0}$ the free-space wave number. Assuming a weak backscattering, the total power backscattered by the waveguide is simply $r_{b} L_{w}$. As in the case of the Payne-Lacey model for the radiative losses (Eq. (10)), also the backscattering is proportional to the variance of the sidewall perturbation $\sigma^{2}$.

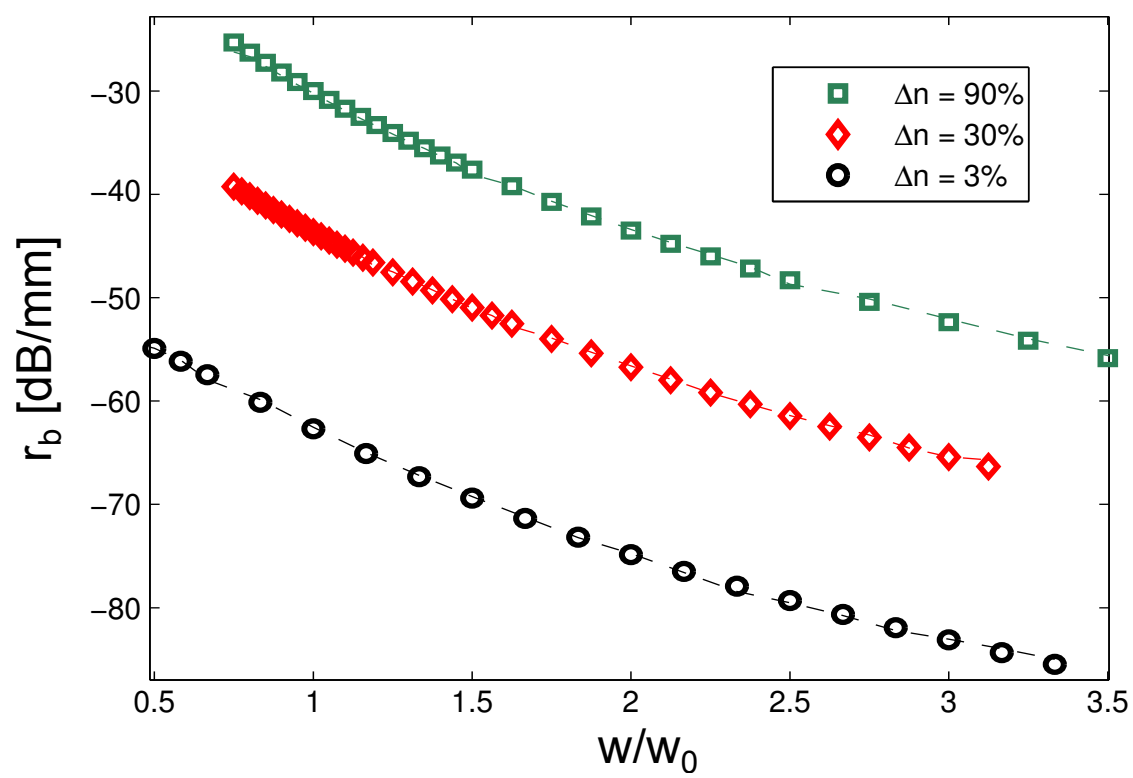

FIGURE 5. Comparison between the backscattered power predicted by the Ladouceur-Poladian model [55] (marks) and the $\left(\partial n_{e f f} / \partial w\right)^{2}$ model (dashed lines). The backscattering is shown as function of the waveguide width normalized to the width of the single mode operation limit $\left(w_{0}\right)$. The same three slab waveguides used in Fig. 3 with the same roughness parameters $\left(\sigma=2 \mathrm{~nm}, L_{c}=50 \mathrm{~nm}\right)$ have considered.

An example of the application of Eq. (20) is provided in Fig. 5 (marks) where the predicted coefficient $r_{b}$ is shown. The three cases refer to the slabs considered in Fig. 3(a) for the TE fundamental mode. The slab width has been normalized to the width limit for single mode waveguides $\left(w_{0}\right)$ for presentation convenience. As expected, a high index contrast increases the sensitivity of the mode to the sidewall imperfection (as in the case of the radiative losses) and consequently the backscattering coefficient, expressed in $\mathrm{dB}$ per millimiter of waveguide. Increasing $\Delta n$ from $3 \%$ to $90 \%$ increases the backscattered light of about $30 \mathrm{~dB} / \mathrm{mm}$, almost independently on $w$.

A different model has been proposed by the authors in [20] considering the roughness profile of the slab sidewalls as a spatial superposition of sinusoidal perturbation of random amplitude, that is a superposition of Bragg gratings. In the small perturbation regime, a sinusoidal sidewall corrugation $\delta w$ over a length 
$L_{w}$ produces a reflection $\kappa L_{w}$ at the Bragg wavelength $\lambda_{B}$, where the coupling coefficient $\kappa$ is

$$
\kappa=\frac{\pi}{\lambda_{B}} \delta n_{e f f}=\frac{\pi}{\lambda_{B}} \frac{\partial n_{e f f}}{\partial w} \delta w
$$

where $\delta n_{\text {eff }}$ is the effective index perturbation associated to $\delta w$. With the same mathematical approach described in Sec. 3.3, the coupling coefficient can be expressed as

$$
\kappa=\frac{\pi}{\lambda_{B}} \frac{\delta w}{w}\left(n_{g}-n_{e f f}\right)
$$

as originally suggested also by Verly et al. for the analysis of distributed feedback [56].

Equation (23) is valid for every waveguide shape and index contrast and implies that the total reflected power generated by the superposition of these infinite contribution depends only on $\partial n_{e f f} / \partial w$ squared or, equivalently, on $\left(n_{g}-\right.$ $\left.n_{e f f}\right)^{2}$

$$
r_{b}=B\left(\frac{\partial n_{e f f}}{\partial w}\right)^{2}=B w^{2}\left(n_{g}-n_{e f f}\right)^{2} .
$$

Similarly to radiative losses also for backscattering a $n_{w}$ model holds, involving a quadratic dependence on $\partial n_{e f f} / \partial w$ instead of the linear dependence given by Eq.(11). With this difference in mind, in the following of the paper we refer to $n_{w}$ model for both radiation losses and backscattering.

Figure 5 shows the comparison of this model with Eq. (20) for the three different slab waveguides (dashed lines). The results are perfectly superposed, suggesting that the two models are essentially equivalent.

With the same arguments of Sec. 3.2, this approach can be extended to laterally confined waveguides and some examples will be provided in the next section. In conclusion, note that the backscattered power is directly proportional to the square of the perturbation (roughness) standard deviation $\sigma^{2}$, as for the radiative losses, and depends on $\left(n_{g}-n_{e f f}\right)^{2}$ while losses have a linear dependance on this parameter.

\subsection{Influence of technologies, polarization state, index contrast, and waveguide shape on the backscattering}

The theoretical treatment presented in the previous section demonstrates that the backscattering is strictly related to the geometry and index profile of the waveguide only through the difference $\left(n_{g}-n_{e f f}\right)^{2}$. In this section the influence of several parameters on the distributed backscatter coefficient $r_{b}$ of a waveguide will be investigated. In particular, the waveguide fabrication technology, the refractive index contrast, the polarization of the light and the waveguide cross section are considered. It is shown also how the models based on the difference $\left(n_{g}-n_{\text {eff }}\right)$ hold independently of all these aspects. The experimental results shown in this section were obtained with the frequency-domain interferometric technique described in $[20,57]$. 


\section{2.a. Technologies}

The technology exploited for the fabrication of integrated devices largely impacts on backscattering (and losses) generated by the waveguides. On one hand, the impact is related to the roughness that arises as consequence of the particular production process, as discussed in Sec. 2 and Tab. 1. On the other side, the refractive index distribution and waveguide shape define the interaction between the propagating mode and the roughness according to the $n_{w}$ model, which is valid for any type of waveguide.

To demonstrate this aspects, four very different optical waveguides are here considered and the results reported. The four waveguides are: a silicon wire in SOI technology $220 \mathrm{~nm}$ thick [20]; a channel SiON waveguide $2 \mu \mathrm{m}$ thick [58]; a rib waveguide with a InGaAsP-based core (thickness $1 \mu \mathrm{m}$ ) on an InP substrate and no cladding [59]; a deeply etched InP-based ridge waveguide with a 360nm-thick multi-quantum well core [60].

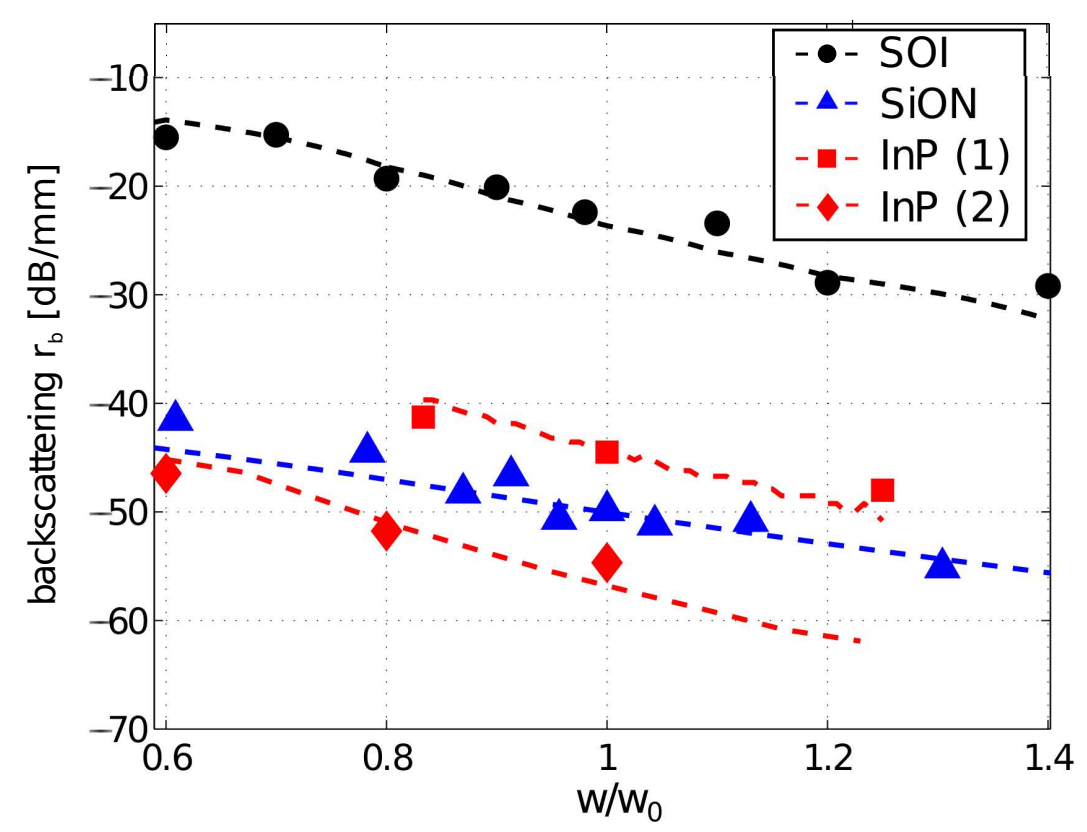

FIGURE 6. Measured (marks) backscattering as function of the normalized waveguide width for several technologies: SOI (black circles) [20], SiON (blue triangles) [58], rib InGaAsP wavegude (red diamonds) [59], ridge InP waveguide (red squares) [60]. The data refer to the TE mode and are numerically fitted (dashed lines) with Eq. (24).

Figure 6 shows the measured backscattered power (marks) as function of the waveguide width (TE input mode) for waveguides in SOI, SiON and InP technologies. The experimental data are fitted with the aforementioned model (dashed lines) based on $\left(n_{g}-n_{e f f}\right)^{2}$. Note that high index contrast SOI waveguides show a backscattering level as high as $30 \mathrm{~dB}$ stronger than the one of low index waveguides. Both InP based waveguides, instead, although uncovered 
are basically weakly guiding and produce a very small backscattering compared to SOI waveguides (even with similar roughness parameters), comparable to low-contrast $\mathrm{SiON}$ waveguides.

TABLE 2. Typical values of losses and backscattering for several technologies with different refractive index contrasts. The waveguide length producing a total backreflection of $-30 \mathrm{~dB}$ is shown as well. Fibre is added as reference.

\begin{tabular}{c|c|c|c|c}
\hline Technology & $\Delta \mathbf{n \%}$ & loss $[\mathbf{d B} / \mathbf{c m}]$ & $\mathbf{r}_{b}[\mathbf{d B} / \mathbf{m m}]$ & L@-30dB \\
\hline \hline $\mathrm{SOI}$ & 140 & 2.5 & -25 & $0.3 \mathrm{~mm}$ \\
$\mathrm{Si}_{3} \mathrm{~N}_{4}$ & 37 & $0.1 \div 2$ & $-30 \div-40$ & $1 \div 10 \mathrm{~mm}$ \\
$\mathrm{SiON}$ & 4.5 & 0.2 & -50 & $10 \mathrm{~cm}$ \\
$\mathrm{InP} / \mathrm{InGaAsP}$ & $3.0 \div 5.0$ & $1.0 \div 2.0$ & $<-40$ & $30 \mathrm{~cm}$ \\
$\mathrm{SiO}_{2}: \mathrm{Ge}$ & $<1$ & 0.1 & $<-60$ & $>1 \mathrm{~m}$ \\
$\mathrm{Fibre}[61]$ & 0.2 & $2 \cdot 10^{-6}$ & $-102($ Rayleigh $)$ & $\infty$ \\
\hline \hline
\end{tabular}

Table 2 summarizes the information of typical index contrast, losses and backscattering for the considered technologies and also for $\mathrm{Si}_{3} \mathrm{~N}_{4}$-based TriPleX waveguides [62] and Germanium-doped glass waveguides. Glass optical fibers are added for reference. It is interesting to note, in the last column, the waveguide length that produces a total backreflection of $-30 \mathrm{~dB}$, corresponding to the Rayleigh scattering in optical fibers. The backscatter gives a negligible contribution to the waveguide attenuation. As an example a backscatter equal to -26 $\mathrm{dB} / \mathrm{mm}$ corresponds to an additional attenuation of $0.1 \mathrm{~dB} / \mathrm{cm}$. However, if for low index contrast waveguides few centimeters can be considered safe, a 300 $\mu \mathrm{m}$ long silicon wire can generate enough reflection to compromise the correct behavior of several circuits.

\section{2.b. Refractive index contrast}

The refractive index contrast plays a fundamental role in defining the propagation characteristic of the waveguide [63] and, clearly, also of the backreflections. In this section, as an example, an SOI $480 \mathrm{~nm}$-wide, $220 \mathrm{~nm}$-thick waveguide is considered $\left(w / w_{0}=0.95\right)$. This waveguide has been realized with three different cladding materials: air (refractive index 1.00), $\mathrm{SiO}_{2}$ (1.45) and SU8 polymeric material (1.58). The refractive index contrast of the waveguide ranges from $200 \%$ and $120 \%$.

Figure 7 shows the backscattering experimental measurements (dots) with the results fitted with the $\left(n_{g}-n_{e f f}\right)^{2}$ model (dashed line). As expected, the sensitivity of the light to the sidewall imperfections and consequently the absolute value of backscattering increases with the contrast. Even in this case the experimental results are well predicted by the model. 


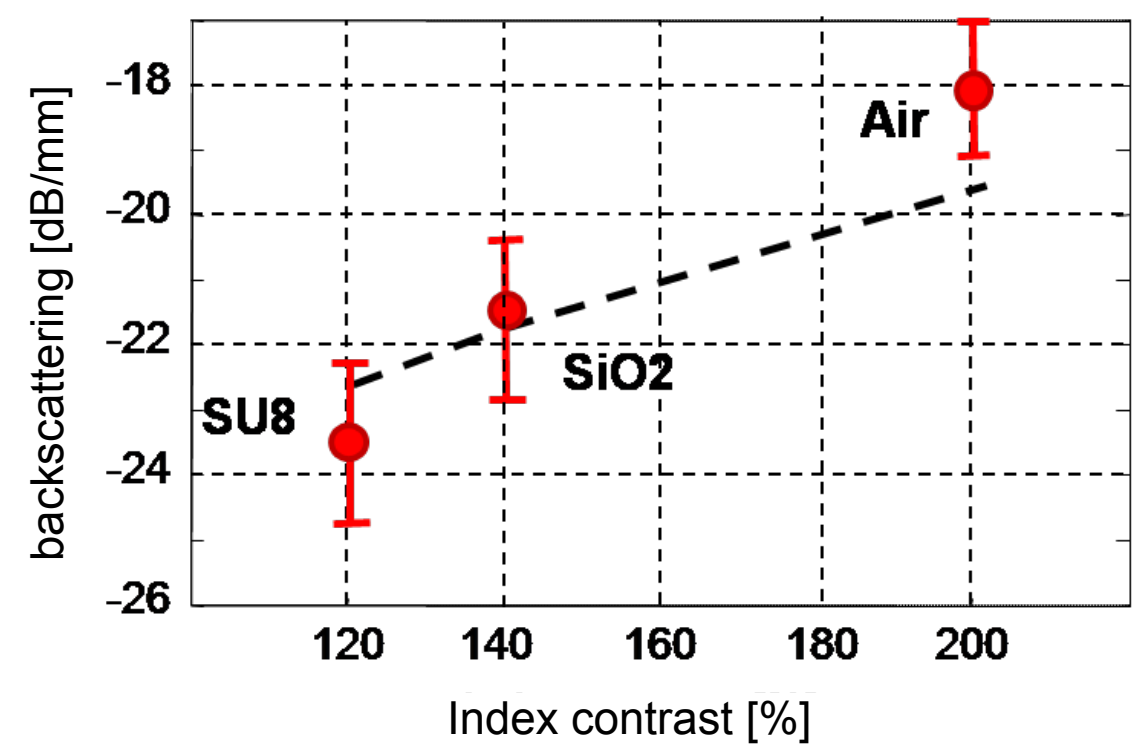

FIGURE 7. Measurements (circles) and model prediction (dashed line) of the backscattering for a SOI waveguide with different cladding material which modifies the refractive index contrast.

\section{2.c. Waveguide shape}

Also the waveguide shape impacts on the backscattering level. Here, the two SOI waveguides shown in Fig. 8, a standard $220 \mathrm{~nm} \times 490 \mathrm{~nm}$ wire and a larger rib waveguide, are considered. This case is very similar to the one experimentally and theoretically investigated by Yap et al. in [52] where a reduction of the losses due to the sidewall roughness is expected moving from a buried waveguide to a shallow etched rib shape due to a diminishing of the interaction of the mode with the sidewall irregularities (there is not any roughness in the horizontal interfaces). The reduction is observed to be proportional to the parameter $s$ in Eq. (13).

The $n_{w}$ model can be used to evaluate the difference in the backscattering between the two waveguides. The wire has $\partial n_{\text {eff }} / \partial w=1.7 \cdot 10^{-3} \mathrm{~nm}^{-1}$ while for the rib $\partial n_{e f f} / \partial w=7 \cdot 10^{-5} \mathrm{~nm}^{-1}$, with a predicted variation of the backscattering of $27 \mathrm{~dB}$. This result confirms the less sensitivity of the rib-shaped waveguides to the sidewall imperfections with respect to deeply-etched structures and the validity of the proposed model.

\section{2.d. Polarization}

Finally, the dependence of the backscatter on the state of polarization of the modes is discussed. This aspect is particularly evident in SOI-based structures, where the behaviour of TE and TM modes is very different. In Fig. 9 the same 


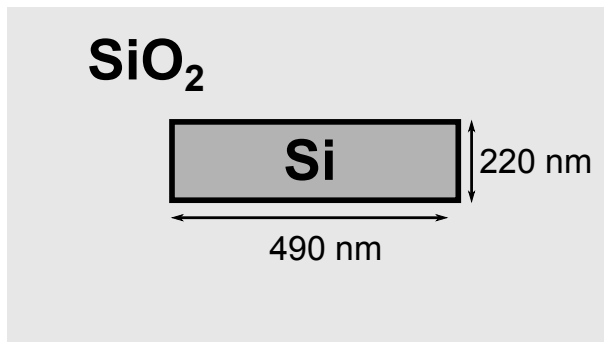

(a)

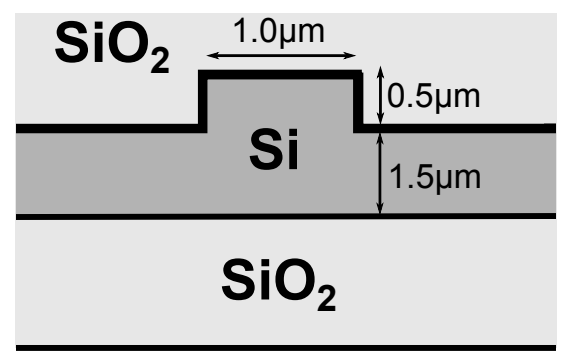

(b)

FIGURE 8. (a) Channel- and (b) rib-shaped SOI waveguide geometrical parameters.

standard channel waveguide of the previous section is considered and the measured backscattered power is shown (marks) as function of the waveguide width for both TE and TM modes. The experimental data are fitted (dashed lines) with Eq. (24), which holds for both modes. TE mode shows a backscatter up to $20 \mathrm{~dB}$ higher with respect to the TM mode and also the dependence on the waveguide width is different and much more sensitive. In general, TM mode results less sensitive to the lateral width variations of the waveguide and is thus indicated for circuits critical to backscattering.

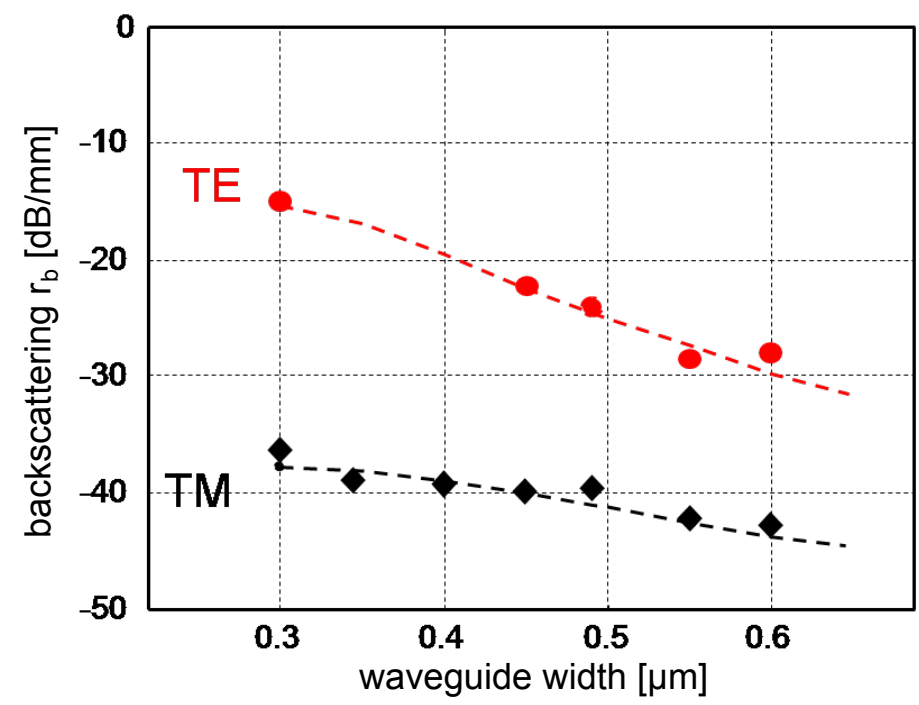

FIGURE 9. Measured backscattering of a channel SOI waveguide for TE (red circles) and TM (black diamonds) modes as function of the waveguide width. Dashed lines are the fit with $n_{w}$ model. 


\section{Roughness and disorder in photonic crystal waveguides}

Although 2D photonic crystal waveguides (PhCWs) can ideally support lossless propagation of the light [6], unavoidable extrinsic scattering loss arises as a consequence of random fabrication imperfections.

Defects are mainly originated by the writing process required to create the $\mathrm{PhC}$ lattice structure. Even by using state-of-the-art electron beam lithography, hole geometry can not be defined with a sub-nm accuracy. Typically, the etch mask is the largest source of disorder and the holes exhibit a rough perimeter, as schematically illustrated in Fig. 10. Roughness of top and bottom surfaces can be usually neglected, because the $\mathrm{PhC}$ membrane is epitaxially grown with an atomic scale control $(0.5-0.6 \mathrm{~nm})$ of the surface quality. Also, the sidewalls are assumed vertical.
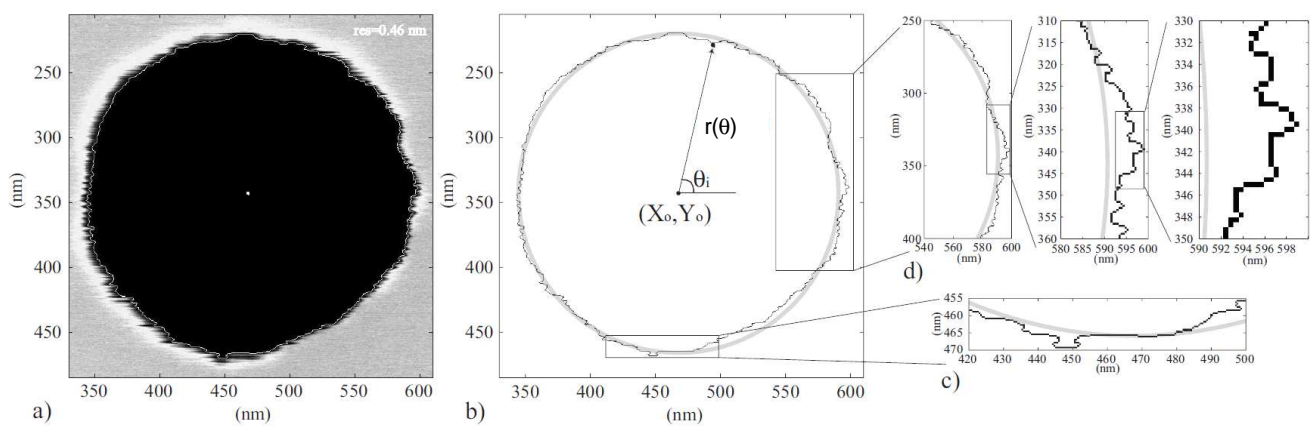

FIGURE 10. (a) SEM picture and (b) model of the hole of a PhCW affected by fabrication imperfections. Both the large scale deviation from the nominal circular shape and the local surface roughness at the boundary can be described assuming a perturbation of the actual radius $r$ around the hole perimeter. Advance modelling can also take into account that (c) at a scale $<2 \mathrm{~nm}$ the hole edge can not be represented by a single valued analytical function $r(\theta)$ and (d) that edge roughness exhibits a fractal behaviour (adapted from [64]).

A comprehensive study of the statistical properties of disorder in $\mathrm{PhC}$ structures was carried out by Skorobogatiy et al. [64]. In this work, the device geometry of PhC lattices was extracted directly from scanning electron microscope (SEM) images, as the one shown in Fig. 10(a), and the main statistical parameters of disordered PhCs were pointed out. In particular, three sets of parameters were identified for a realistic modelling of $\mathrm{PhC}$ structures:

- a first set of parameters includes coarse properties of the hole shape, such as radius $r$, ellipticity, and other low angular momenta components, which can be deliberately designed or be the result of fabrication tolerances. Among these, radius variations $\delta r(\theta)$ along the angular coordinate $\theta$ [see Fig. 10(b)] give the most relevant contribution to disorder; 
- a second set of parameters describes roughness at the hole boundary on a nanometer scale. Roughness can be modelled by using the root mean square (RMS) perturbation amplitude $\sigma$, that is the deviation with respect to the ideal dielectric permittivity profile, and by introducing the concept of correlation length $L_{c}$. In analogy to the case of regular waveguides (see Sec. 2), in PhCWs $L_{c}$ is defined as the distance over which the occurrence of defects is correlated to one another [40]. Typical values of $\sigma$ and $L_{c}$ are 2-3 $\mathrm{nm}$ and 40-50 nm, respectively, even though some results suggest that higher values of correlation length must be considered (see Sec. 6.4);

- the last set of parameters describes random displacements of the hole position from the ideal periodic lattice. In the presence of symmetry breaking elements (such as waveguides), $\mathrm{PhC}$ lattices typically exhibit an anisotropic position disorder along the transversal and longitudinal directions.

All these features contribute to create what is generally referred to as structural disorder in PhCWs. Comparing the statical parameters of different holes belonging to the same structure, it was found that these contributions are usually uncorrelated from one hole to another.

As a good approximation, disorder can be modelled as a radius perturbation $\delta r(\theta)$ along the perimeter of the hole [see Fig. 10(b)], according to the following expression [65]

$$
\left\langle\delta r\left(\theta_{m}\right) \delta r\left(\theta_{m^{\prime}}^{\prime}\right)\right\rangle=\sigma^{2} \exp \left(\frac{-r\left|\theta_{m}-\theta_{m^{\prime}}^{\prime}\right|}{L_{c}}\right) \delta_{m, m^{\prime}},
$$

where $m$ is the hole index and the Kronecker function $\left(\delta_{m, m^{\prime}}=1\right.$ for $m=m^{\prime}$ and $\delta_{m, m^{\prime}}=0$ for $m \neq m^{\prime}$ ) takes into account the absence of correlation between two different holes. Although this model does not include some features of the disorder profile, such as the small scale $(<2 \mathrm{~nm})$ surface folding visible in Fig. 10(c) and the fractal behaviour of Fig. 10(d), this disorder model has the advantage of describing the $\mathrm{PhC}$ disorder by means of only two parameters $\left(\sigma\right.$ and $L_{c}$ ) that can be directly related to experiments. Therefore, this model is widely used to study the scattering effects and, ultimately, to predict the propagation loss of $\mathrm{PhCWs}$.

\section{Loss in photonic crystal slab waveguides}

In the last decade, much effort has been devoted to the problem of realistically modelling disorder-induced scattering loss in PhCWs. Due to the inherent high complexity of the structure and to the large number of disorder degrees-offreedom, estimating the loss of $\mathrm{PhCWs}$ is more challenging than in the case of regular optical waveguides.

This section specifically addresses this problem, presenting a comprehensive overview of the main results achieved in the field. In Sec. 6.1 a theoretical model 
is presented, which can be used to describe disorder-induced scattering in generic $\mathrm{PhCWs}$; then we discuss in details, through numerical and experimental results, the role of the group velocity (Sec. 6.2), of the mode shape (Sec. 6.3) and of the correlation length (Sec. 6.4) on the extrinsic scattering loss of PhCWs.

Some analogies with the results presented in Secs. 3 and 4 for regular waveguides are found and discussed in Sec. 13.

\subsection{Theoretical model}

Disorder-induced scattering loss in PhCWs have been studied by using several approaches, including for instance vectorial eigenmode expansion (EME) [66], coupled mode theory (CMT) [67], guided mode expansion (GME) [68, 69], Fourier-Bloch-mode method (FBMM) [70,71], and Bloch-mode expansion (BME) [72-74]. First works were limited to understand the behaviour of specific and simplified structures, made for instance of 2D infinitely long cylinders $[75,76]$ or layered structures [66], with analysis carried out through systematic numerical investigations [77].

The first attempt to provide a generic theoretical model to describe the effects of disorder in arbitrary PhCWs was proposed by Hughes et al. [78]. In this work a photon Green-function-tensor formalism was employed to derive explicit expressions for extrinsic optical scattering loss in PhCWs. In the Hughes' model, loss in PhCWs were demonstrated to arise from two dominant scattering processes: backscattering, that is light scattering from a forward propagating mode into a backward propagating mode, and out-of plane scattering into radiation modes above the light line.

The backscattering loss $\alpha_{b}$ and the radiation loss $\alpha_{r}$ per unit cell are given by the following expressions,

$$
\left\langle\alpha_{b}\right\rangle=\left(\frac{a \omega}{2 v_{g}}\right)^{2} \iint d \mathbf{r} d \mathbf{r}^{\prime}\langle\Delta \varepsilon(\mathbf{r})\rangle\left\langle\Delta \varepsilon\left(\mathbf{r}^{\prime}\right)\right\rangle\left[\mathbf{e}_{k}(\mathbf{r}) \cdot \mathbf{e}_{k}(\mathbf{r})\right]\left[\mathbf{e}_{k}^{*}\left(\mathbf{r}^{\prime}\right) \cdot \mathbf{e}_{k}^{*}\left(\mathbf{r}^{\prime}\right)\right] e^{i 2 k\left(x-x^{\prime}\right)}
$$

and

$$
\left\langle\alpha_{r}\right\rangle=\frac{a \omega}{v_{g}} \iint d \mathbf{r} d \mathbf{r}^{\prime \prime}\left\langle\Delta \varepsilon\left(\mathbf{r}^{\prime}\right)\right\rangle\left\langle\Delta \varepsilon\left(\mathbf{r}^{\prime \prime}\right)\right\rangle \mathbf{e}_{k}^{*}\left(\mathbf{r}^{\prime}\right) e^{-i k x^{\prime}} \cdot \operatorname{Im}\left[\overrightarrow{\mathbf{G}}_{r a d}\left(\mathbf{r}^{\prime}, \mathbf{r}^{\prime}, \omega\right)\right] \cdot \mathbf{e}_{k}\left(\mathbf{r}^{\prime \prime}\right) e^{i k x^{\prime \prime}}
$$

whose full analytical derivation can be found in [65]. The expected value $\langle\cdots\rangle$ indicates that these expressions predict the average loss of many nominally identical structures made of a single lattice cell. In eqs. (26) and (27), $a$ is the lattice period of the $\mathrm{PhCW}, \Delta \varepsilon(\mathbf{r})$ is the disorder function, that is the difference between the ideal and the actual (disordered) spatial profile of the dielectric permettivity, and $\mathbf{e}_{k}(\mathbf{r})$ is the electric field of the ideal Bloch mode propagating along the $x$ direction with wave vector $k$ and group velocity $v_{g}$. As discussed in Sec. 5, roughness on the surface of the holes is typically the dominant source of scattering $[79,80]$, so that $\Delta \varepsilon(\mathbf{r})$ can be assumed as a nonzero function only on the hole boundaries. The radiation mode Green function $\overrightarrow{\mathbf{G}}_{r a d}\left(\mathbf{r}^{\prime}, \mathbf{r}\right.$ ", $\left.\omega\right)$ in Eq. 
(27) is a tensor, whose component $G_{r a d, i j}\left(\mathbf{r}, \mathbf{r}^{\prime}, \omega\right)$ provides the $i$-component of the electric field induced at a position $\mathbf{r}$ ' by a $j$-polarized dipole placed at $\mathbf{r}$ " and oscillating at an angular frequency $\omega$.

It is important to underline here, that eqs. (26) and (27) are derived through an incoherent single-scattering approach that does not take into account multiple scattering events. In $\mathrm{PhCW}$ s this condition is typically fulfilled at high group velocity, but as the propagation of the light is slowed down, phenomena associated with multiple scattering dramatically affect the propagation properties of the light and must be included in the model. In this regime a coherent scattering approach is thus required [81], as discussed in Sec. 7.

Although the calculation of the loss coefficients $\left\langle\alpha_{b}\right\rangle$ and $\left\langle\alpha_{r}\right\rangle$ from Eqs. (26) and (27) is not straightforward, these expressions are very informative to point out the main features of scattering processes occurring in PhCWs. The product $\left\langle\Delta \varepsilon\left(\mathbf{r}^{\prime}\right)\right\rangle\left\langle\Delta \varepsilon\left(\mathbf{r}^{\prime \prime}\right)\right\rangle$ indicates a quadratic dependence of both radiation loss and backscattering on the disorder function, that is on the $\sigma^{2}$ parameter of Eq. (25), in agreement with other theoretical models [68]. A detailed analysis of the dependence of the backscatter and radiation loss on the disorder parameters and on the properties of the propagating field is discussed in detail in following of this section.

\subsection{Group velocity dependence}

One of the main results of the Hughes' model is that it clearly points out the role of the group velocity $v_{g}$ of the light in the scattering processes occurring in PhCWs. Both backscatter and radiation loss increase at smaller $v_{g}$, because of the higher interaction time of the light with the structural disorder. Expressing this dependence in terms of the group index $n_{g}=c / v_{g}$, out-of-plane radiative loss $\alpha_{r}$ is found to have approximately a linear dependence on $n_{g}$, while backscattering loss $\alpha_{b}$ scales according to $n_{g}^{2}$. According to this model, total extrinsic loss in PhCWs can be simply expressed as [82]

$$
\alpha=\alpha_{r}+\alpha_{b}=c_{1}^{\prime} n_{g}+c_{2}^{\prime} n_{g}^{2},
$$

where the scaling factors $c_{1}^{\prime}$ and $c_{2}^{\prime}$ can be calculated through eqs. (26) and (27).

Since the Hughes' model does not make any assumptions on the spatial distribution of the holes in the $\mathrm{PhCW}$, the model applies to arbitrary structures. Therefore, it can be used to study scattering processes in conventional PhCWs with a regular lattice, such a W1 PhCWs [83], where a line of holes is removed to create the waveguide, as well in structures where one or more rows of holes are shifted [84] and/or modified [85] to engineer the dispersion behaviour of the waveguide.

To give an example, let us consider the dispersion-engineered waveguide of Fig. 11. As shown in the top-view SEM photograph of Fig. 11(a), the waveguide is designed starting from a conventional W1 waveguide and by laterally shifting the first and second rows of holes adjacent to the defect line by distances $s_{1}$ and 
(a)

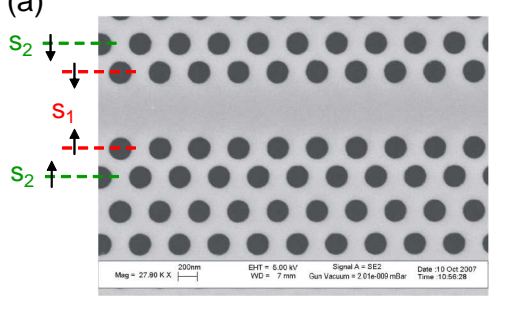

(b)

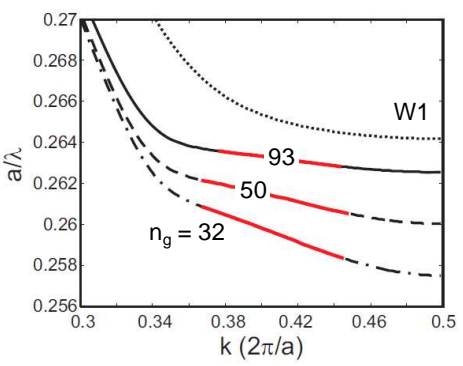

(c)

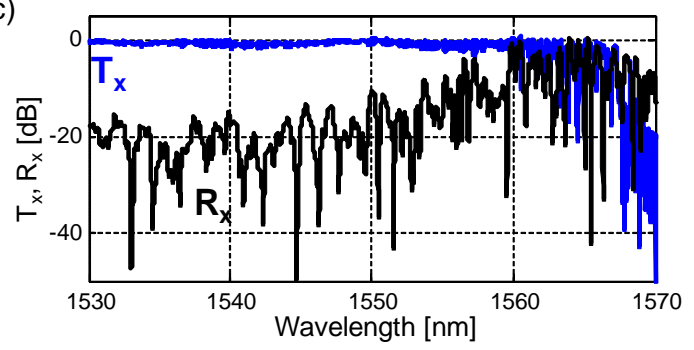

(d)

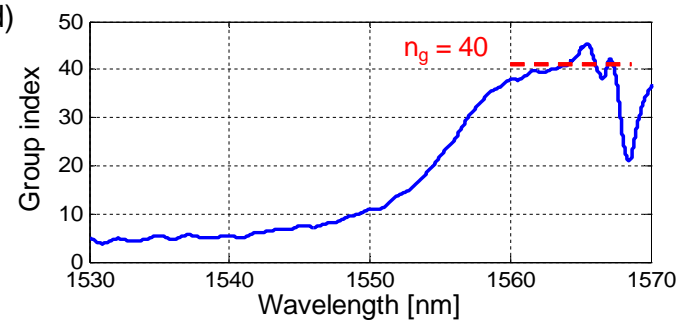

FIGURE 11. (a) Top-view photograph of a dispersion-engineered $\mathrm{PhCW}$ obtained by laterally shifting the first and second rows of holes of a W1 PhCW by symmetric displacements $s_{1}$ (red) and $s_{2}$ (green). (b) Calculated dispersion curves for the fundamental mode of dispersion engineered PhCWs with the following displacement parameters: $s_{1}=-0.13 a, s_{2}=0$ (dashdotted curve), $s_{1}=-0.1225 a, s_{2}=0.045 a$ (dashed curve), and $s_{1}=-0.1 a$, $s_{2}=0.085 a$ (solid curve). The thick solid red line indicates the flat band slow light region with group index $n_{g}$. The dotted line indicates the dispersion relation of a W1 waveguide. [84] (c) Measured normalized transmission $\left(T_{x}\right.$, blue) and backscatter $\left(R_{x}\right.$, black) of a silicon membrane dispersion engineered PhCW with $s_{1}=-48 \mathrm{~nm}, s_{2}=16 \mathrm{~nm}, a=410 \mathrm{~nm}$, and $r=0.286 a$. (d) Measured group index of the PhCW of (c), exhibiting a flat group index of about 40 between $1560 \mathrm{~nm}$ and $1567 \mathrm{~nm}$. ((a) reproduced from [84], (b) is adapted from [84], (c) and (d) are reproduced from [82])

$s_{2}$, respectively (positive shift indicated hole displacement towards the waveguide centre) [84]. This is one of the strategies proposed in literature to modify the position of the band-gap guided mode with respect to the index guided mode [86], thus creating a region of low-dispersion slow light with a nearly constant $n_{g}$ versus wavelength and an optimized bandwidth. Simulations in Fig. 11(b) show the dispersion curves of the fundamental mode of engineered PhCWs with several combinations of the displacement parameters $s_{1}$ and $s_{2}$. A region with a linear dispersion relation (thick red line) is created providing a nearly constant group index $n_{g}=32$ (dash-dotted curve), 50 (dashed curve), and 93 (solid curve). Other approaches to tailor the dispersion curve of $\mathrm{PhCWs}$ and to optimize the group index bandwidth products (GBPs) have been proposed in literature $[85,86]$ and can exploit also dispersion compensation in chirped PhCWs [87].

Fig. 11(c) shows the measured normalized transmission ( $T_{x}$, blue curve) and back-reflection $\left(R_{x}\right)$ of a PhCW engineered according to the approach depicted 
in Fig. 11(a)-(b). The waveguide is realized on a $220 \mathrm{~nm}$ thick silicon membrane with a $\mathrm{PhC}$ lattice period $a=410 \mathrm{~nm}$ and hole radius $r=0.286 a$. The waveguide is engineered with parameters $s_{1}=-48 \mathrm{~nm}, s_{2}=16 \mathrm{~nm}$, and is $180 \mu \mathrm{m}$ long. The fabrication processes, which is described in detail in Refs. [82,88], is based on electron beam lithography of a $220 \mathrm{~nm}$ SOI wafer, followed by reactive ion etching (RIE) and a selective removal of the buried oxide underneath the silicon layer. By using this process, PhCWs with a disorder of less than $2 \mathrm{~nm}$ RMS [89] and propagation loss as low as $5 \mathrm{~dB} / \mathrm{cm}$ in the fast light regime were demonstrated.

The backscattering and the group index of the waveguide, the latter shown in Fig. 11(d), were measured by using coherent optical frequency domain reflectometry (OFDR) [20,90]. Alternatively, Fourier-transform spectral interferometry [91] or optical low-coherence reflectometry (OLCR) [92,93] can be used for group index measurements. Approaching the transmission band-edge, located at wavelength of about $1568 \mathrm{~nm}$, the group index linearly, but steeply increases from a value below $10(\lambda<1550 \mathrm{~nm})$ up to nearly $40(\lambda<1560 \mathrm{~nm})$, followed by a low-dispersion region with a constant $n_{g}$ of about 40 in the $1560 \mathrm{~nm}<\lambda<$ $1568 \mathrm{~nm}$ spectral region. In agreement with theoretical predictions, stating a $n_{g}^{2}$ scaling of backscattering, the backreflected power steeply increases moving toward the band edge. In Fig. 11(c), backreflection remains around $-20 \mathrm{~dB}$ in the wavelength range between $1530 \mathrm{~nm}$ and $1550 \mathrm{~nm}$, but it becomes comparable to the transmitted power above $1560 \mathrm{~nm}$. In this region, backscattering dominates over out-of-plane loss and becomes the main sources of loss. This makes also multiple scattering events become more significant [82,94], this generating deep oscillations of both the transmission and backscattering spectral response. These phenomena, that cannot be explained with the incoherent scattering model of eqs. (26) and (27), are discussed in detail in Sec. 7. Other experimental observations demonstrate that the scaling rules of backscatter and radiation loss versus $n_{g}$ predicted by the Hughes's model hold up to relatively high group index (up to 30$)[79,94,95]$, but they may break down at a higher values.

Besides the extrinsic scattering losses associated with disorder, which are distributed along the waveguide, it is worthwhile to mention here also the problem of light injection in a $\mathrm{PhCW}$ operating in a high group index regime [96]. A group index mismatch between the $\mathrm{PhCW}$ and the input/output ridge waveguides generates coupling loss and concentrate reflections at the $\mathrm{PhCW}$ terminations. Therefore, suitable impedance matching regions are needed to optimize the coupling efficiency. These can be obtained, for instance by using an adiabatic taper $[97,98]$, by inserting a fast light section of $\mathrm{PhCW}$ between the ridge waveguide and the slow light section of photonic crystal [84,99-101]. The use of an intermediate fast-light region was also exploited to reduce the loss due to stitching errors, when electron beam lithography is used to write a PhCW [102]. 


\subsection{The influence of the optical mode shape}

As mentioned in Sec. 6.2, the dependence of radiation loss and backscattering on $n_{g}$ is only approximate and in some circumstances it can dramatically underestimate the actual loss of PhCWs. To point out this issue, we experimentally quantified the backscattering versus $n_{g}$ of different kind of PhCWs. Figure 12a shows the measured enhancement factor of the backscattering, that is the backscattering normalized to a reference level in the low group-index regime $\left(n_{g}\right.$ equal to about 5). The wavelength dependence of the measured group index is shown in Fig. 12(b). Two PhCWs are considered, which were engineered according to the design of Fig. 11 in order to have a low-dispersion region at a group index of about 30 (black curve) and 40 (blue curve). The backscattering curves of both waveguides follows the $n_{g}^{2}$ model (dashed line) up to a group index of about 25 and 35, respectively, that is up to the beginning of the low dispersion region. At a higher wavelength, the group index flattens, but the backscattering steeply increases, diverging abruptly from the $n_{g}^{2}$ model. This behavior is evidently not consistent with a simple $n_{g}^{2}$ model, that would have predicted no change in the backscatter in the low-dispersion region.

The physical mechanism underneath this steep increase of the backscattering curve is that the shape of the Bloch mode propagating through the structure strongly depends on the group index itself $[103,104]$. The incoherent scattering model proposed by Hughes can predict this effect, provided that the evolution of $\mathbf{e}_{k}(\mathbf{r})$ versus $n_{g}$ is taken into account in eqs. (26) and (27), that is in the computation of the scattering parameters $c_{1}^{\prime}$ and $c_{2}^{\prime}$ of Eq. (28).

Figure 13(a) shows the simulated dispersion curve (blue curve) of the fundamental mode of an engineered $\mathrm{PhCW}$ with design parameters $s_{1}=-48 \mathrm{~nm}$ ans $s_{2}=16 \mathrm{~nm}$, realized with a 220 -nm-thick Si suspended membrane. The lattice period and the nominal hole radius are $a=410 \mathrm{~nm}$ and $r=112 \mathrm{~nm}$, respectively. Dispersion engineering produces a region of low-dispersion slow light (highlighted in cyan) with a nearly constant group index of about 40 . The intensity profile $\left|\mathbf{e}_{k}(\mathbf{r})\right|^{2}$ of the Bloch mode at the three wavevectors marked by the three red circles are shown in Fig. 13(b). At small group indices the mode is strongly confined in the waveguide and the optical field weakly interacts with the holes boundaries. As the wave vector increases, propagation enters the slowlight region, and the electric field spreads over the first row of holes where it interacts strongly with the surface disorder.

This strong variation of the field distribution at the vanishing dispersion point was theoretically addressed by Petrov et al. [104] as the cause of a sharp increase of the backscattering in dispersion engineered PhCWs. The physical reason underneath this behaviour is that the point of vanishing dispersion results from the anticrossing of two different modes [86]. Away from the anticrossing point, the mode profile changes slowly with frequency and disorder-induced scattering changes slowly with the group index. Around the anticrossing point, the mode shape abruptly changes even though the group index remains almost constant, so 

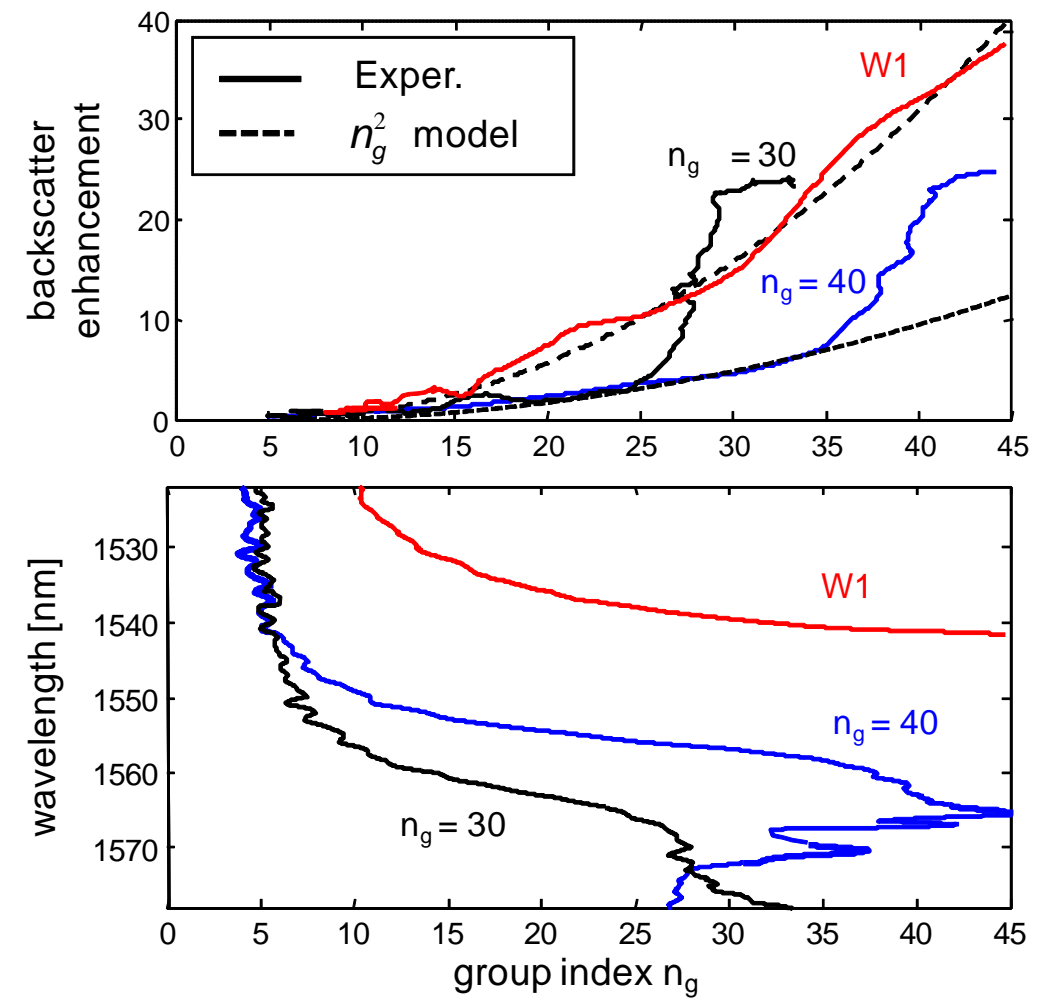

FIGURE 12. (a) Measured backscatter enhancement versus $n_{g}$ for several PhCWs: the black and the blue curves indicates a dispersion engineered waveguide designed to have a low-dispersion region at a group index of 30 and 40, respectively, while the red curve indicates a W1 waveguide. Dashed lines show the $n_{g}^{2}$ model for both kind of waveguides. (b) Measured group index of the three PhCWs shown in (a).

that in this regime the scattering loss scales differently from $n_{g}$ for out-of-plane scattering or $n_{g}^{2}$ for backscattering.

The interaction of the optical field with the structural disorder can be quantified by calculating the integral of $\left|\mathbf{e}_{k}(\mathbf{r})\right|^{2}$ across the surfaces of the holes. Results shown in Fig. 13(c) point out that, for a given group index $n_{g}$, the modes of two different $\mathrm{PhCW}$ can interact very differently with disorder. In the case of the engineered waveguide of Fig. 13a (green, solid), a sharp increase in the concentration of the electric field in the disorder region is observed around the engineered region of $n_{g}=40$. In contrast, in a conventional W1 waveguide $\left(s_{1}=\right.$ $\left.s_{2}=0\right)$ the mode distribution evolves slowly with wave vector and does not exhibit this phenomenon (blue, dashed). This behaviour nicely agrees with the experimental results shown in Fig. 12 for the W1 waveguide. For a W1 PhCW, the smoother evolution of the mode shape versus $n_{g}$ is associated with a slower increase of the backscattering, with no sharp transitions in the high group index regime. This behaviour is more in line with a $n_{g}^{2}$ model [dashed curve in 
(a)

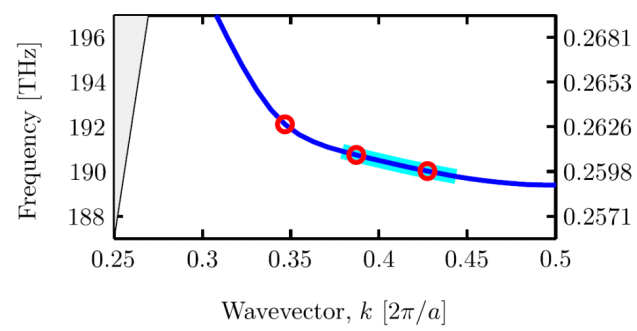

(b)

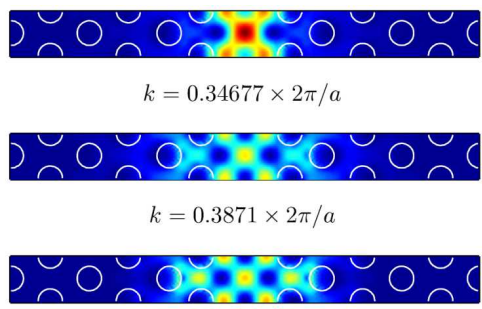

$k=0.42742 \times 2 \pi / a$

(c)

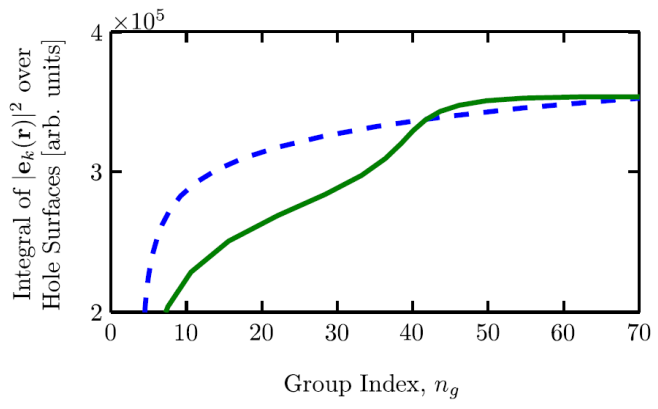

FIGURE 13. (a) Dispersion relation (blue curve) of the fundamental mode of an engineered PhCW $\left(s_{1}=-48 \mathrm{~nm}, s_{2}=16 \mathrm{~nm}, \mathrm{a}=410 \mathrm{~nm}\right.$, and hole radius $r=112 \mathrm{~nm})$. The region of near-constant group index $\left(n_{g}=40\right)$ is highlighted in cyan. (b) Electric field distribution of the Bloch mode for the three wave vectors marked by red circles in (a). (c) Integral of the Bloch mode intensity $\left|\mathbf{e}_{k}(\mathbf{r})\right|^{2}$ over the hole surfaces as a function of group index $n_{g}$ for the dispersion engineered structure shown in (a) (blue dashed curve), and for a conventional W1 waveguide (green, solid curve). (images are reproduced from $[80])$

Fig. 12(a)]. The higher backscatter of the $\mathrm{W} 1$ waveguide in the low $n_{g}$ regime $\left(n_{g}<25\right)$ is also consistent with the higher interaction of the optical field with the structural disorder shown in Fig. 13(c).

These results clearly demonstrate that in $\mathrm{PhCWs}$ backscattering is not related to the group index only. This means that there are some degrees of freedom to reduce scattering loss, at a given group index, by properly engineering the mode interaction with the holes boundaries [105].

\subsection{Correlation length}

Another parameter that strongly affects the properties of the scattering processes occurring in $\mathrm{PhCWs}$ is the correlation length $L_{c}$ of disorder, that is the distance over which defects are correlated to one another [40].

In order to clarify the role of the correlation length, it is convenient to express the total extrinsic loss of a $\mathrm{PhCW}$ as [82]

$$
\alpha=c_{1} \gamma n_{g}+c_{2} \rho n_{g}^{2},
$$

where the parameters $\gamma$ and $\rho$ are associated with radiation loss and backscatter 
loss, respectively. It should be noted that the coefficients $c_{1}$ and $c_{2}$ are not related the optical field distribution, but only to technological parameters, to the degree of disorder, and to the perturbation of the dielectric contrast $\Delta \varepsilon$ of the PhCW [71]. Therefore, in Eq. (29) the disorder $\left(c_{1}, c_{2}\right)$ and mode shape $(\gamma, \rho)$ contributions are separated, thus enabling the possibility of study the loss-issue by suitably tuning the mode shape.

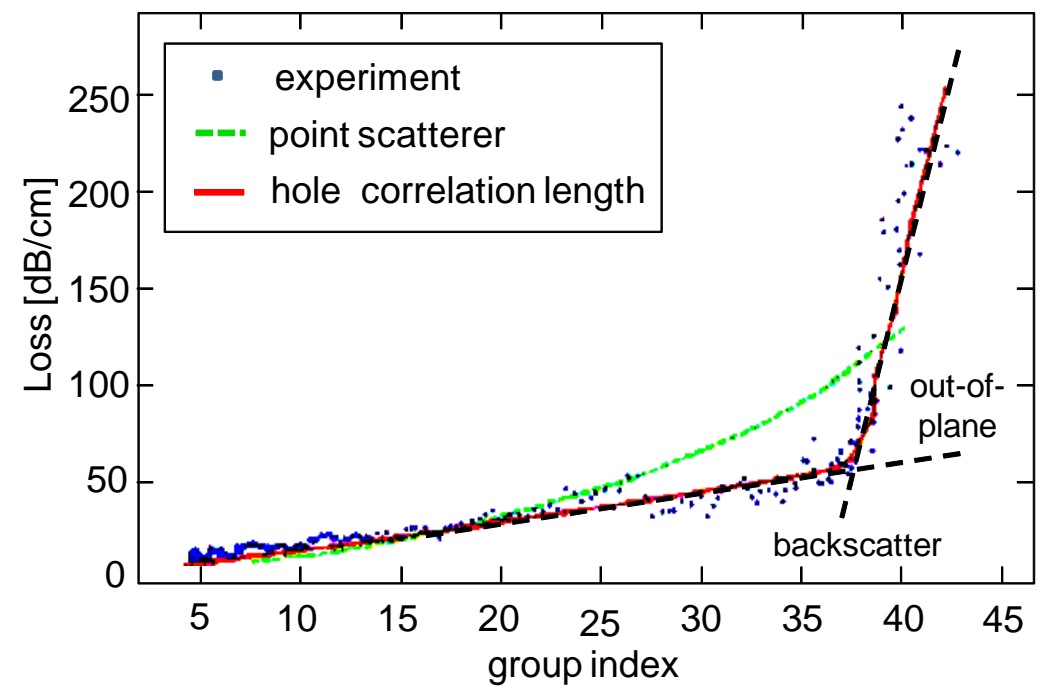

FIGURE 14. Total loss of dispersion engineered $\mathrm{PhCW}$ s with a group index of $n_{g}=38$ in the low-dispersion region (displacement parameters $s_{1}=-48$ $\mathrm{nm}, s_{2}=16 \mathrm{~nm}$ ). The green dashed curve is the numerical fit obtained by assuming a zero correlation length $\left(L_{c}=0\right)$, while the red solid curve assumes a coherence length equal to the entire hole perimeter (adapted from [82]).

As shown in [82], the coeffients $\rho$ and $\gamma$ can be derived by modelling each scattering point as a radiating dipole [106], and by taking into account that radiation loss implies the coupling with a continuum of radiation modes, whereas backscattering occurs into a single backpropagating mode. The mode shape dependent backscattering parameter is given by [82,106]

$$
\rho=\sum_{n}\left|\int_{L_{c}} \mathbf{E}_{T} \mathbf{E}_{T}+\left(\varepsilon_{1}\right)^{-1} \mathbf{D}_{N} \mathbf{D}_{N} d \mathbf{r}\right|^{2},
$$

while the out-of-plane coefficient is

$$
\gamma=\sum_{n}\left|\int_{L_{c}} \mathbf{E}_{T}+\left(\varepsilon_{1}\right)^{-1} \mathbf{D}_{N} d \mathbf{r}\right|^{2}
$$

In eqs. (30) and (31), $\mathbf{E}_{T}$ is the electric-field component tangential to the hole surface and $\mathbf{D}_{N}$ is the displacement-field component normal to the hole surface, and $\varepsilon_{1}$ is the dielectric constants of the material constituting the photonic crystal. 
To the scope of our analysis, the main information given by eqs. (30) and (31) is that the holes of a unit cell are divided into $n$ parts of length $L_{c}$, which corresponds to the correlation length of the lattice imperfections measured along the boundary of a hole. Since $L_{c}$ is the length over which both integrals have to be calculated, it represents a critical parameter for an accurate estimation of the coefficients $\rho$ and $\gamma$. The relevance of the correlation length in the determination of the backscattering coefficient $\rho$ was also addressed in [107], where a CMT model was proposed predicting a linear increase of $\rho$ versus $L_{c}$ for guided modes near the band edges.

To illustrate the impact of the correlation length on the total loss of a $\mathrm{PhCW}$, let us consider the result shown in Fig. 14. Blue markers indicate the total loss measured on dispersion engineered $\mathrm{PhCWs}$ designed to exhibit a constant group index of $n_{g}=38$ over a wavelength range $\Delta \lambda=8 \mathrm{~nm}\left(s_{1}=-48 \mathrm{~nm}, s_{2}=16 \mathrm{~nm}\right)$. The green dashed curve is the behaviour predicted by Eq. (29) by assuming $L_{c}=$ 0 , that is by considering the losses as generated by independent point scatterers, whose contributions add incoherently [104]. A much better agreement between the model and the experimental results is obtained by assuming a $L_{c}$ equal to the entire hole perimeter (red solid curve). In particular, this assumptions describes well the abrupt change in the loss curve at $n_{g}=38$, that is in the low-dispersion engineered region. Moreover, the model confirms that the linear dependence of the loss at $n_{g}<35$ is due to the $n_{g}$ dependence of the out-of-plane loss, because in this regime backscattering is almost suppressed [see also Fig. 11(d)]; the steep increase of the backscattering shown in Fig. 12a is responsible for the dramatic increase in the total loss around $n_{g}=38$. The validity of the model assuming $L_{c}$ equal to the entire hole perimeter has been also demonstrated in the case of W1 waveguides [82].

This result suggests that all the scattering events generated in a $\mathrm{PhCW}$ by any source of disorder (surface roughness, hole displacement and deformation) add coherently along the surface of the entire hole. This also implies that the correlation length in photonic crystals is larger than the value of $20-50 \mathrm{~nm}$, which is typically assumed for silicon photonic wires, and which had been assumed for PhCWs in previous works $[78,80]$.

\section{Multiple scattering}

Multiple scattering in a generic optical medium occurs when the light is scattered more than once before outgoing the structure. This happens when the mean-free path $l$ of the electromagnetic wave becomes shorter than the sample length $L$. In our study of scattering processes in regular and $\mathrm{PhC}$ waveguides, we have implicitly considered so far the case of rare scattering events $(l>>L)$, this regime being typically referred to as coherent [108,109], ballistic [71] or dispersive regime [110]. In this condition, a $\omega-k$ dispersion relation describing the propagation properties of the light is well defined, thereby making $\mathrm{PhCW}$ behave similarly to regular waveguides. For instance, as discussed in Sec. 10, the transmission 


\section{(a)}
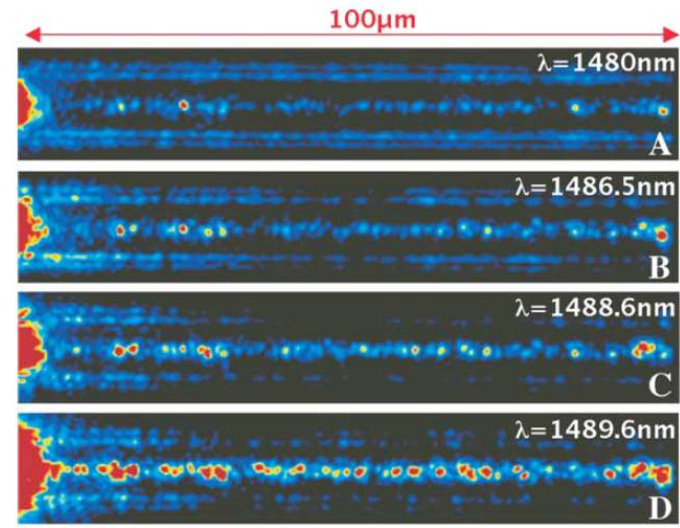

\section{C}
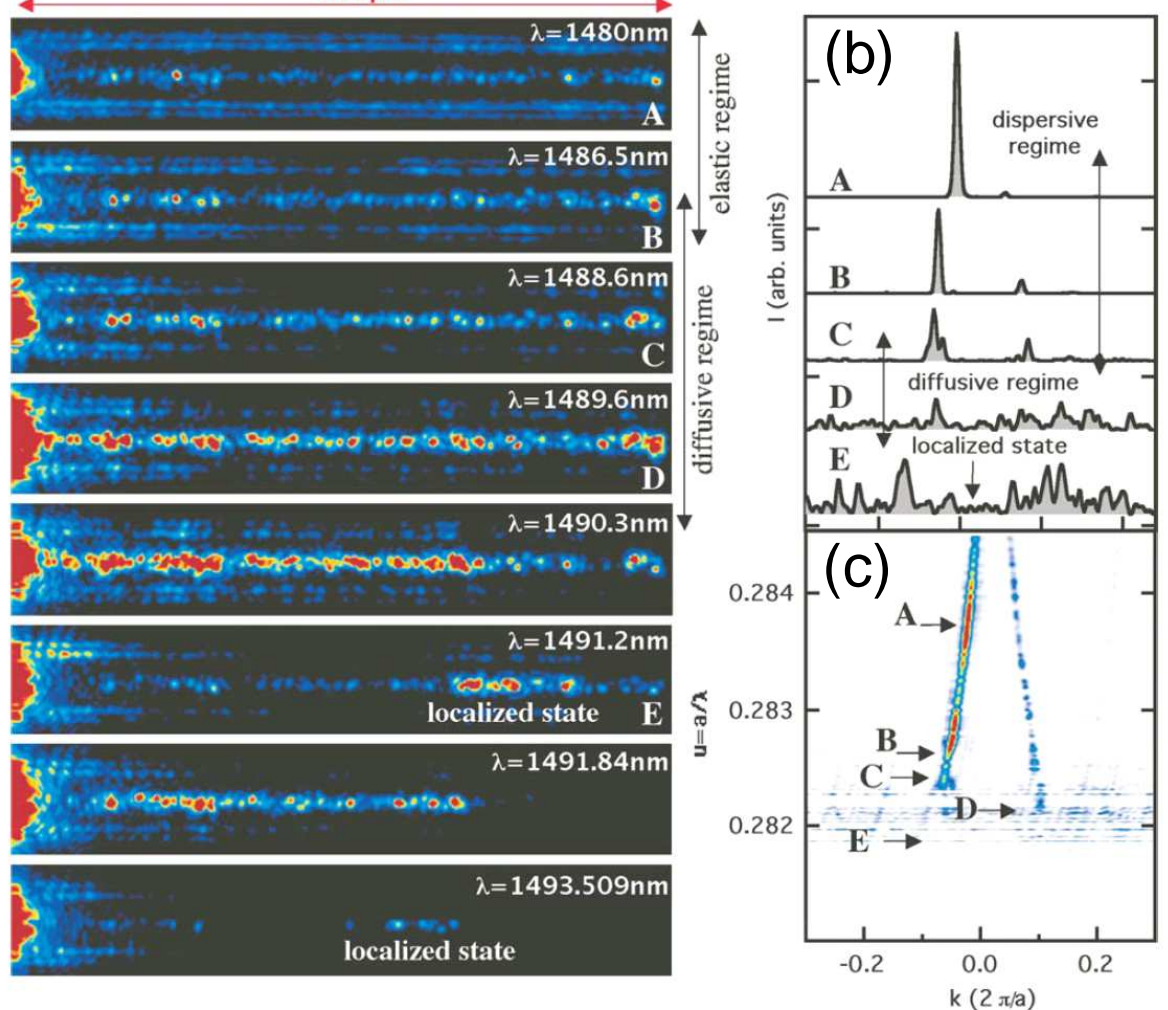

$\lambda=1493.509 \mathrm{~nm}$

localized state

FIGURE 15. Experimental observation of the transition of the light transport through a disordered $\mathrm{W} 1 \mathrm{PhCW}$ across different regimes: dispersive (A to $\mathrm{C}$ ), diffusive ( $\mathrm{C}$ to $\mathrm{D}$ ), and localization ( $\mathrm{E}$ and beyond): (a) real-space images of the infrared field radiated at the top surface of the waveguide at different wavelengths; (b) angular spectrum profiles measured at different regimes; (c) measured dispersion diagram of the waveguide. (reproduced from [110])

and the reflection of the light propagation through disordered $\mathrm{PhCWs}$ in the dispersive regime and in regular waveguides affected by surface roughness share the same statistics properties.

Conversely, in the multiple scattering regime $(l<<L)$, conventional wave equations may bread down and the description of light propagation requires a diffusive model [111]. In contrast to classical diffusion, the wave diffusive regime is associated with interference effects with backscattered field contributions, as observed in the coherent backscattering of the light in disordered scattering media $[112,113]$. In this regime a dispersion relation between the angular frequency $\omega$ and the wave vector $k$ may be not defined, the wave vector $k$ can not be considered anymore a good quantum number [110], and several phenomena arise that can not find their counterpart in regular waveguides. 


\subsection{From dispersive (single-scattering) to diffusive (multiple-scattering) regime}

When the group velocity of the light propagating through a $\mathrm{PhCW}$ is reduced, structural disorder may be responsible for a transition from the dispersive regime to the diffusive regime. This effect was experimentally investigated by Le Thomas et al. through a direct measurement of the dispersion curve of a $\mathrm{PhCW}$ in $k$ space [110]. In this work, a high numerical aperture optical Fourier-space imaging technique, providing the $2 \mathrm{D}$ angular spectrum of the field emitted from the surface of a $\mathrm{PhCW}$, was used to determine the phase velocity, the dispersion curve and the spatial frequency spectrum of the modes excited in the structure.

Figure 15 shows the results obtained on a W1 waveguide fabricated on a 260-nm-thick InP suspended membrane with a $\mathrm{PhC}$ lattice period $a=440 \mathrm{~nm}$, designed to operate at a wavelength of about $1550 \mathrm{~nm}$. At shorter wavelengths $(\lambda<1488 \mathrm{~nm}$, patters A to B) a pure dispersive regime occurs, which is characterized by an almost uniform intensity pattern extended along all the waveguide length (a), a narrow spatial frequency spectrum (b), and a well defined dispersion curve (c), thereby implying a well defined group velocity $v_{g}$. The low intensity symmetric trace in the dispersion curve (c) corresponds to the counter-propagating mode excited by the back reflection at the cleaved facet of the output waveguide.

When the normalized frequency $u=a / \lambda$ approaches the band edge (1488 nm $<\lambda<1490 \mathrm{~nm}$, patterns $\mathrm{C}$ to $\mathrm{D}$ ), the effect of the disorder becomes significant as a result of the slowing down of the light. Multiple-scattering modifies the optical intensity pattern along the waveguide with the formation of localized states (a), the linewidth of the angular spectrum broadens, as a results of the decrease of the mean-free path $l$ due to the random spatial dephasing of the field, and is characterized by the formation of speckle features (b). In the diffusive regime, the dispersion curves steeply breaks down (c), spectral transmission is characterized by large fluctuations and even though an energy transport velocity $v_{E}$ can be defined, it strongly deviate from $v_{g}$ [114-116].

Although Fig. 15 clearly shows that the propagation properties of the light dramatically change from the dispersive to the diffusive regime, no direct information on the efficiency of the light transmission, in term of propagation loss, is contained. Actually, even though in the pure diffusive regime the spectral correlations are too weak to generate a clear dispersion curve, the light transmission through the waveguide can be still efficient, in contrast to the strongly spatially localized regime, which appears at frequencies located below the ideal band edge $(\lambda>1490 \mathrm{~nm}, \mathrm{E}$ and beyond). Mazoyer et al. argued that, in the pure localization regime, the propagation of optical pulses is so distorted that neither the group-velocity nor the energy velocity are meaningful quantities [116]. The propagation loss issue in the multiple-scattering regime is specifically addressed in Secs. 7.2 and 7.3. 
(a)

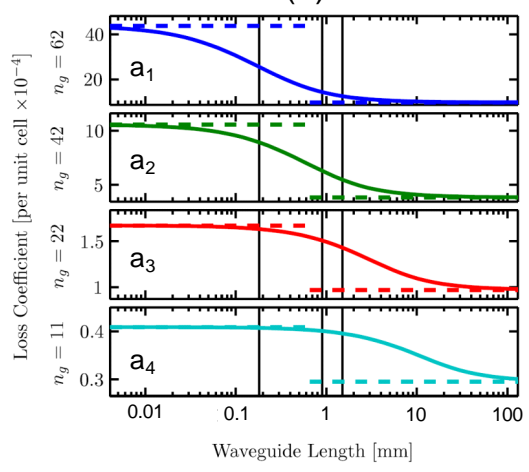

(b)

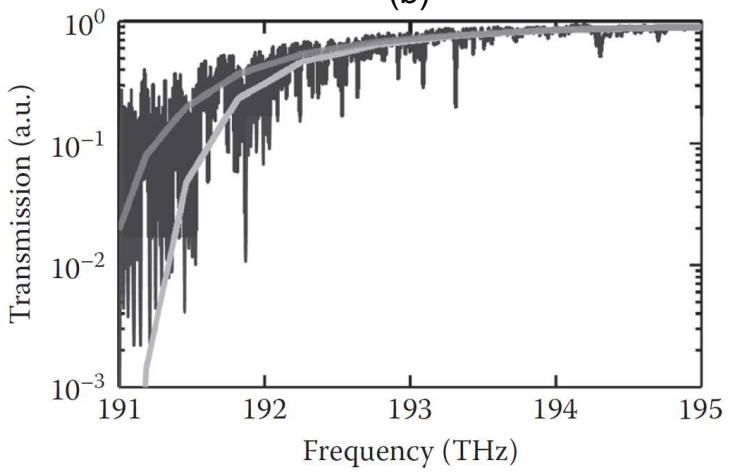

FIGURE 16. (a) Calculated average loss per unit cell (solid curves) of a $220-\mathrm{nm}$ silicon membrane dispersion engineered $\mathrm{PhCW}\left(s_{1}=-48 \mathrm{~nm}, s_{1}=\right.$ $16 \mathrm{~nm}, a=410 \mathrm{~nm}, r=112 \mathrm{~nm})$ as a function of the waveguide length for different group index regime: $n_{g}=62$ ( $a_{1}$, blue curve), $n_{g}=42\left(a_{2}\right.$, green curve), $n_{g}=22$ ( $a_{2}$, red curve), and $n_{g}=11$ ( $a_{4}$, cyan curve). In each plot, the short and the long waveguide limits are indicated with the upper and lower dashed curves. (reproduced from [80]) (b) Comparison between the measured transmission spectrum of a W1 waveguide (black curve) and the spectrum calculated by using the Beer-Lambert model (light gray) and the incoherent multiple scattering model. (reproduced from [65])

\subsection{Loss in the multiple scattering regime}

In the multiple scattering regime, not only the dispersion curve of a $\mathrm{PhCW}$ undergoes an abrupt transition, but the propagation loss itself exhibits some features that have no counterparts in regular waveguides. Patterson et al. demonstrated that one of the most striking consequences of multiple scattering is the break down of the Beer-Lambert law [80]. Classically, the Beer-Lambert law states that the intensity of a wave propagating through an optical waveguide of length $L$ evolves as

$$
P(L)=P(0) \exp (-\alpha L),
$$

where $P(0)$ is the intensity at the input of the waveguide and the propagation loss coefficient $\alpha$ is a constant. The Beer-Lambert model strictly applies to any waveguide where the light scattered or absorbed in any point along the structure never reaches the output port. However, in a system with strong backscattering, such as a $\mathrm{PhCW}$ in the low group-velocity regime, there is a certain probability for the light to be backscattered multiple times before being transmitted. This makes the Beer-Lambert model fails. Similar results were demonstrated by Wang et al. [106] for a two-dimensional system.

In the multiple scattering regime, the average loss per unit cell can be extrapolated from eqs. (26) and (27) by using an incoherently averaged coupled mode approach [80]. The result is the following set of coupled equations for the 
intensity of the forward $\Psi_{f}(x)$ and backward $\Psi_{b}(x)$ modes, respectively,

$$
\begin{aligned}
& \frac{d \Psi_{f}(x)}{d x}=-\left(\left\langle\alpha_{b}\right\rangle+\left\langle\alpha_{r}\right\rangle\right) \Psi_{f}(x)+\left\langle\alpha_{b}\right\rangle \Psi_{b}(x), \\
& \frac{d \Psi_{b}(x)}{d x}=\left(\left\langle\alpha_{b}\right\rangle+\left\langle\alpha_{r}\right\rangle\right) \Psi_{b}(x)-\left\langle\alpha_{b}\right\rangle \Psi_{f}(x) .
\end{aligned}
$$

It should be noted that this model deals with the intensity of the modes, that are coupled by the expected values of the radiation loss $\left\langle\alpha_{r}\right\rangle$ and of the backscattering loss $\left\langle\alpha_{b}\right\rangle$ calculated through eqs. (26) and (27). Being derived from an incoherent scattering approach, eqs. (33) and (34) can not describe coherent interference effects associated with multiple scattering, such as the deep oscillations close to the band-edge of the transmission spectrum of a $\mathrm{PhCW}$. Nonetheless they can effectively give information on the average value of the transmission spectrum.

Fig. 16(a) shows the effective average loss per unit cell of a dispersion engineered $\mathrm{PhC}$ waveguide (designed according to the scheme of Fig. 11(a) with displacement parameters $s_{1}=-48 \mathrm{~nm}$ and $s_{2}=16 \mathrm{~nm}$ ) as a function of total waveguide length for four different frequencies. For short waveguides, the effective loss coefficient is found to be equal to $\alpha_{b}+\alpha_{r}$, in agreement with the Beer-Lambert model (upper dashed limit), while in longer waveguides, multiple scattering makes the effective loss per unit cell decrease to $\alpha_{r} \sqrt{1+2 \alpha_{b} / \alpha_{r}}$ (lower dashed limit). Note that moving at higher group indices, the difference between the short and the waveguide limits increases. Obviously, if $\alpha_{b}<<\alpha_{r}$, the two limits degenerate and the multiple scattering method agrees with the Beer-Lambert method for all waveguide lengths.

Fig. 16(b) shows the comparison between the measured (black curve) and calculated transmission spectrum of a W1 waveguide. Loss calculation is performed by extrapolating to a waveguide with a length $L=1.5 \mathrm{~mm}$ the Beer-Lambert model (light gray) and the multiple scattering model (dark gray). The disorder parameters used in the calculation are $\sigma=3 \mathrm{~nm}$ and a correlation lenght $L_{c}$ $=40 \mathrm{~nm}$. Approaching the band edge, the Beer-Lambert model overestimates the transmission loss, while the multiple scattering model better reproduces the experimentally observed roll-off. Similar results have been found also in the case of dispersion engineered waveguides [80]. As mentioned before, this incoherent scattering model can predict the expected value of the transmission averaged over many nominally identical disordered waveguides, but it can not describe the Fabry-Pérot fringes in short frequency range of the PhCW transmission spectrum. These effects are inherently associated with coherent interference effects originated by disorder-induced coherent scattering, and will be discussed in Sec. 7.3 .

The results shown in this section agree with the work by Baron et al. [117], confirming that the classical exponential-damping law for the propagation loss of periodic optical waveguides holds only as far as the group velocity remains quite high. When the group velocity of the light reduces, a stationary damping-rate 
regime is found only for infinitely long waveguides, while for short waveguides a transient regime with much larger local damping rates is observed in general, in agreement with the results of Fig. 16(a).

\subsection{Coherent scattering in PhCWs}

The incoherent theoretical model developed by Hughes's to describe extrinsic loss due to disorder induced scattering (see Sec. 6.1) provides the expected value of the transmission properties of $\mathrm{PhCWs}$, that is the result that one would obtain by averaging over a large number of nominally identical samples. In the multiple scattering regime, this approach can predict the failure of the Beer-Lamber law (Sec. 7.2), but cannot explain the presence of narrow band resonances that have been observed experimentally when the transmission spectrum of the $\mathrm{PhCW}$ approaches the band edge $[92,118]$.

To model this effects, Patterson and Hughes extended the coupled mode approach used in Ref. [80], by developing a non-perturbative theory of coherent optical scattering over multiple periods of a disordered waveguide [81]. In this model, the Green function approach is included in a coupled mode formalisms in such a way that the complex amplitudes of the forward-backward (and radiated) coupled modes are calculated at each point along the waveguide. With respect to the incoherent model, the coupling coefficients are calculated by a 3D modelling of individual disordered waveguides instead of a statistical average over many disordered waveguides.

In the coherent scattering model the electric field propagating in the structure along the $x$ direction is described by [81]

$$
\mathbf{E}(\mathbf{r}, \omega)=E_{0}\left[\mathbf{e}_{k}(\mathbf{r}) \Psi_{f}(x) e^{i k x}+\mathbf{e}_{k}^{*}(\mathbf{r}) \Psi_{b}(x) e^{-i k x}\right]+\mathbf{E}_{r a d}(\mathbf{r}, \omega),
$$

where $\Psi_{f}(x)$ and $\Psi_{b}(x)$ are the slowly-varying envelopes of the forward and backward propagating field, $E_{0}$ is a constant amplitude factor, and $\mathbf{e}_{k}(\mathbf{r})$ is the normalized Bloch mode propagating with wave vector $k$. The term $\mathbf{E}_{\text {rad }}(\mathbf{r}, \omega)$ takes into account contributions from radiation modes. Propagation is described by the following set of coupled mode equations,

$$
\begin{aligned}
v_{g} \frac{d \Psi_{f}(x)}{d x} & =i c_{f f}(x) \Psi_{f}(x)+i c_{f b}(x) e^{-i 2 k x} \Psi_{b}(x)+i C_{f, r}(x), \\
-v_{g} \frac{d \Psi_{b}(x)}{d x} & =i c_{b b}(x) \Psi_{b}(x)+i c_{b f}(x) e^{i 2 k x} \Psi_{f}(x)+i C_{b, r}(x),
\end{aligned}
$$

where

$$
c_{f f}(x)=c_{b b}(x)=\frac{\omega a}{2} \iint \mathbf{e}_{k}^{*}(\mathbf{r}) \cdot \mathbf{e}_{k}(\mathbf{r}) \Delta \varepsilon(\mathbf{r}) d y d z
$$

is the self-coupling coefficient of one mode into itself and

$$
c_{b f}(x)=c_{f b}^{*}(x)=\frac{\omega a}{2} \iint \mathbf{e}_{k}(\mathbf{r}) \cdot \mathbf{e}_{k}(\mathbf{r}) \Delta \varepsilon(\mathbf{r}) d y d z
$$


(a)

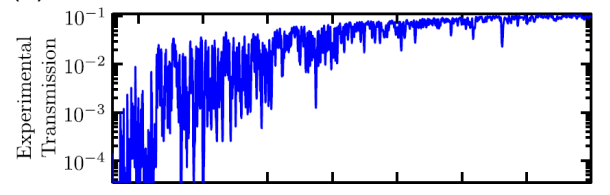

(b)

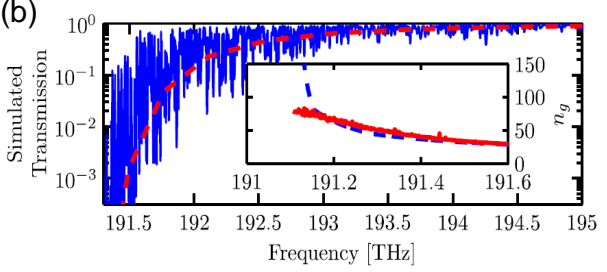

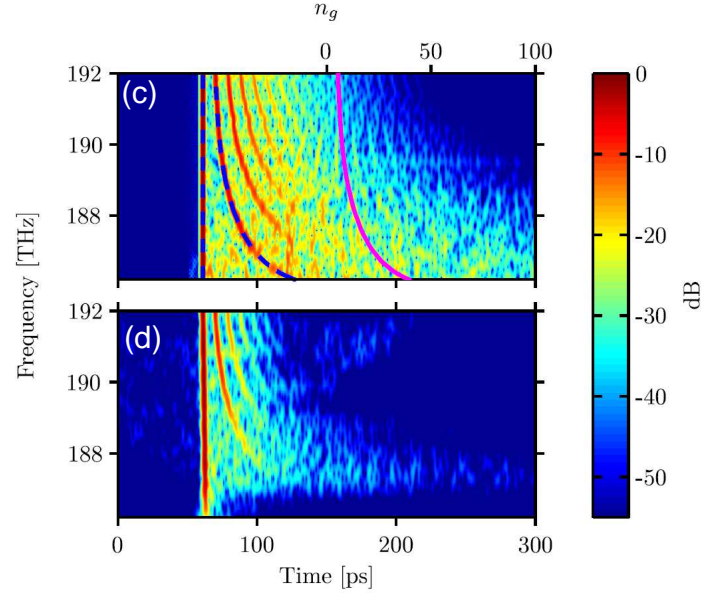

FIGURE 17. Comparison between (a) the measured transmission spectrum of a W1.1 PhCW and (b) the theoretical spectrum calculated by using the incoherent (dashed, red line) and coherent (solid, blue) scattering approach. The red curve in the inset shows that actual value of the group index does not diverge like the ideal value (dashed, blue). Comparison between the simulated (c) and experimental (d) time-frequency reflectance map of a $250 \mu \mathrm{m}$ long W1.1 waveguide. In the simulated map, the left blue dashed line indicates the injection time of the light and the right blue dashed curve indicates the expected round trip time in a disorder-free structure. The magenta line shows the group index $n_{g}$ on the top scale. ((a) e (b): reproduced from [103])

is the coupling coefficient into the counterpropagating mode, with $\Delta \varepsilon(\mathbf{r})$ being the disorder function, that is the difference between the ideal and the actual profile of the dielectric permettivity. A similar integral definition applies to the terms $C_{f, r}(x)$ and $C_{b, r}(x)$ taking into account the coupling into the radiation modes above the light line [81].

The coherent scattering model nicely describes the behaviour of the transmission spectrum of a $\mathrm{PhCW}$ in the vicinity of the band edge, as shown in Fig. 17 by the comparison between simulated (a) and experimental results (b). The measurement was performed on a W1.1 PhCW waveguide fabricated on a 265 $\mathrm{nm}$ thick GaAs suspended membrane, with a PhC lattice $a=400 \mathrm{~nm}$ and hole radius $r=0.27 a$. The waveguide is $1.1 \sqrt{3} a$ wide and $1.5 \mathrm{~mm}$ long. The $\mathrm{rms}$ roughness $\sigma$ and the correlation length $L_{c}$ are $3 \mathrm{~nm}$ and $40 \mathrm{~nm}$, respectively. Approaching the band edge, the average transmission spectrum drops down according to the incoherent loss model (dashed red curve), but many sharp oscillations appear making the transmission vary rapidly versus frequency by orders of magnitude. These Fabry-Pérot-like resonances arises from multiple scattering events between disorder sites and are well reproduced by the coherent scattering model without any fit parameters.

A deeper physical insight into the multiple-scattering phenomena occurring 
in $\mathrm{PhCWs}$ is provided by time-frequency reflectance maps (TFRM), showing the intensity of the backreflected signal as a function of time (or optical length) and frequency. Fig. 17 show the comparison between the TFRM calculated by using the coherent scattering method (a) and measured on the waveguide of Fig. 17(b). Experimentally, the map can be generated by using the complex reflectance of the waveguide, which can be measured through optical low coherence interferometry (OLCR) $[92,93,119]$ or coherent optical frequency domain reflectometry (OFDR) [20]. The dashed blue lines in the simulated map indicate the reference time, that is the time of arrival of the light reflected at the front facet of the sample, and the round-trip time, that is the time of arrival from the rear facet of the sample. Multiple reflections between the front and rear facets are visible, whose round trip time increases at lower frequencies, because of the group velocity reduction. Approaching the bandgap, the sharp reflection at the rear facet of the samples disappears (at frequencies $<188 \mathrm{THz}$ ) because multiple scattering arises and produces random Fabry-Pérot-like resonances along the waveguide. The good agreement between the simulated and experimental TFRM demonstrates that the coherent scattering model provides a realistic description of the coherent scattering mechanism occurring in a $\mathrm{PhCW}$. In Sec. 10, TFRMs are also used to analyze the statistic properties of light propagation in dispersion engineered PhCWs.

A direct measurement of the local optical field propagating through $\mathrm{PhCWs}$ was made by Engelen et al. [94], showing that the modal pattern becomes strongly irregular in the multiple scattering regime. In this experiment, a phasesensitive near-field microscope [120] was used to monitor the light intensity inside a chirped $\mathrm{PhCW}$ [see Fig. 18(a)], that is a waveguide where the hole radius is gradually increased along the propagation direction $[87,121,122]$. The benefit of using a chirped waveguide is that, as the hole size increases, the waveguide mode in the dispersion relation shifts to higher frequencies. Therefore, the position in the waveguide for which a specific group velocity occurs changes with wavelength, thereby making it possible to explore the effects of unintentional disorder in different group velocity regimes. A chirped W1 PhCW was fabricated on a suspended silicon membrane (210 nm thickness) with a hexagonal lattice of air holes with a pitch $a=456 \mathrm{~nm}$. The hole radius increases linearly from $142 \mathrm{~nm}$ to $150 \mathrm{~nm}$ over a length of 300 lattice periods, with typical local fabrication variations in diameter of approximately $3 \mathrm{~nm}$.

Figure 18b shows the measured amplitude of the optical field along the $\mathrm{PhCW}$ (the light is incident from the left) at a decreasing normalized angular frequency $\omega$ (measured in units $2 \pi c / a$ ) of $0.2973\left(b_{1}\right), 0.2961\left(b_{2}\right)$, and $0.295\left(b_{3}\right)$. As the frequency of the light approaches the band edge, the cut-off region, that is the region where the waveguide no longer allows propagation and the light of backreflected, moves leftwards toward the waveguide input. Along the waveguide the intensity of the field gradually increases because of the group velocity reduction along the chirped $\mathrm{PhCW}$, but close to cut-off region the field pattern broadens laterally and becomes strongly irregular, indicating the presence of multiple 
(a)

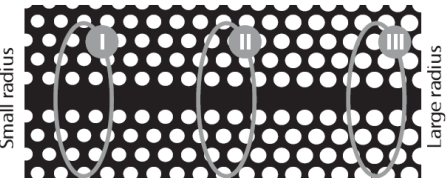

(b)

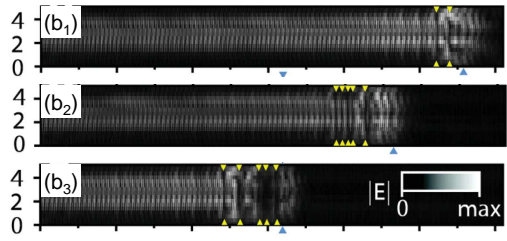

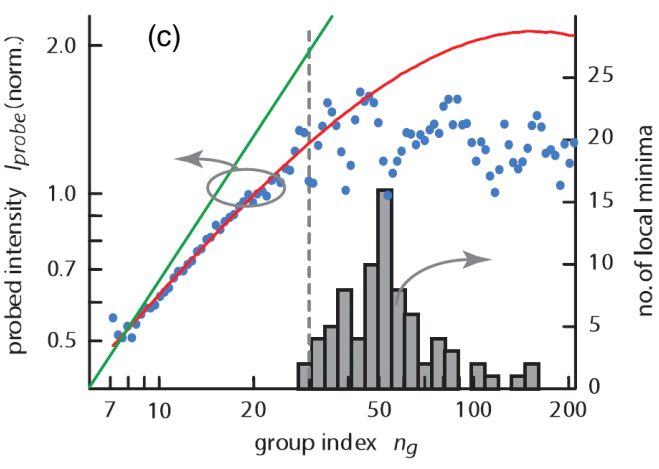

FIGURE 18. (a) Schematic of the chirped PhCW: the radius of the holes linearly increases from the input section (left) to the output section (right, so that the dispersion curve of the $\mathrm{PhCW}$ shift along the waveguide. (b) Near field measurement of the electric field amplitude along the chirped waveguide at a normalized angular frequency $\omega=0.2973\left(b_{1}\right.$, region I), $\omega$ $=0.2961\left(b_{2}\right.$, region II), and $\omega=0.2950\left(b_{3}\right.$, region III). (c) Normalized intensity as a function of the group index. The green curve shows the expected without losses. The red line represents the best fit by using the measured data points with $n_{g}$ below 30 only resulting in a $n_{g}^{2}$ curve. The histogram depicts at what $n_{g}$ local intensity minima were observed, indicative of multiple scattering. (adapted from [94])

scattering events. Even though the group velocity around the cut-off regions of $\left(b_{1}\right)-\left(b_{3}\right)$ is comparable $(c / 30)$, the intensity pattern is different for each frequency, because it is generated by different local realization of the disorder.

The measured optical intensity $I_{\text {probe }}$, averaged over 25 effective realizations and normalized to the intensity measured at $n_{g}=20$, is show in Fig. 18(c) as a function of the group index. For $n_{g}$ between 7 and 30, the intensity increases with the reduction of group velocity, the divergence with respect to the ideal linear scaling (green line) [123] being associated with propagation loss. The red curve demonstrates that in this $n_{g}$ range the intensity in the waveguide is well described by a Beer-Lambert law

$$
I(x+d x)=I(x) \exp \left[-A n_{g}^{2}(x) d x\right],
$$

where $A$ is a constant and the damping exponent scales with the square of the group index. In the cut-off regione $\left(n_{g}>30\right)$, the optical intensity shows an irregular pattern and cannot be described by Eq. (40). The histogram in Fig. 18(c) shows the occurrence of amplitude minima in the near-field measurements, pointing out that the group index at which the fitted red curve no longer matches measured data coincides with the first appearance of local minima. Above this group index, propagation cannot be described by using a perturbative approach because multiple scattering effects strongly modify the propagation properties, generating highly localized resonances. Note that the transition between the two propagation regimes highlighted in Fig. 18(c) corresponds to the transition from 
the dispersive to diffusive regimes discussed in Sec. 7.1. Finally, the group index at which this transition occurs depends on the quality of the fabrication: the lower the structural disorder of the waveguide, the higher the group index up to which dispersive regime can extend.

\section{Statistics of waveguide backscattering}

It has been shown in the previous sections how backscattering originates as consequence of the interaction between the propagating mode(s) and a random perturbation of the refractive index profile of the waveguide. Some effects of this interaction can be observed also in the direct propagating direction as a non-deterministic fluctuation of the group delay experienced by the light propagating through an optical waveguide [124]. In particular, a broadening of the probability distribution of the group delay around an expected average value when the length of the waveguide increases has been observed suggesting the presence of a disturbance generated by the random interaction with the sidewall roughness (forward-scattering). In any case, this fluctuation remains generally sufficiently small (maximum standard deviation around $2 \%$ ) and a strong deterministic component dominates the forward-propagating signal. This is not the case for the backward-propagating light where a prevailing component is missing (neglecting strong lumped reflections) and only a random-generated signal remains. The study of the statistical characteristics of this signal is then of great importance for a correct understanding of the phenomena and an accurate modelling of realistic optical waveguides and integrated circuits, similarly to the investigations carried out on the statistical nature of the Rayleigh scattering in optical fibers $[125,126]$.

The random nature of the backscattering is related to both the actual position along the waveguide at which the reflection occurs and the value of the (complex) 'reflection coefficient' locally generated by the width perturbation. It has been proved in [127] that rough waveguides behave as single scattering systems, independently of shape, size, and refractive index contrast of the waveguide, and independently of the light polarization state. Referring to the scheme in Fig. 19(a), the properties of this system can be described by means of a complex function $h_{R}(z)$, which represents the backscatter generated by a waveguide section of length $d z$ placed at a distance $z$ from the beginning of the waveguide (being the total length of the waveguide equal to $L_{w}$ ).

The complex amplitude $h_{R}(z)$ of the backscattering can be measured with the same frequency-domain interferometric technique mentioned above [20,57], which allows the measurement of both amplitude and phase of the local backscattering distributed along a waveguide. By Fourier transforming this function the spectrum $H_{R}(\lambda)$ and the power spectral density $\left|H_{R}(\lambda)\right|^{2}$ of the cumulative backscattering at the input of the waveguide can be retrieved. The previously

used power backscattering coefficient is linked to this quantity by the relation $r_{b}=\left\langle\left|H_{R}(\lambda)\right|^{2}\right\rangle_{\lambda}$, where $\langle\cdots\rangle_{\lambda}$ indicates a spectral average. 


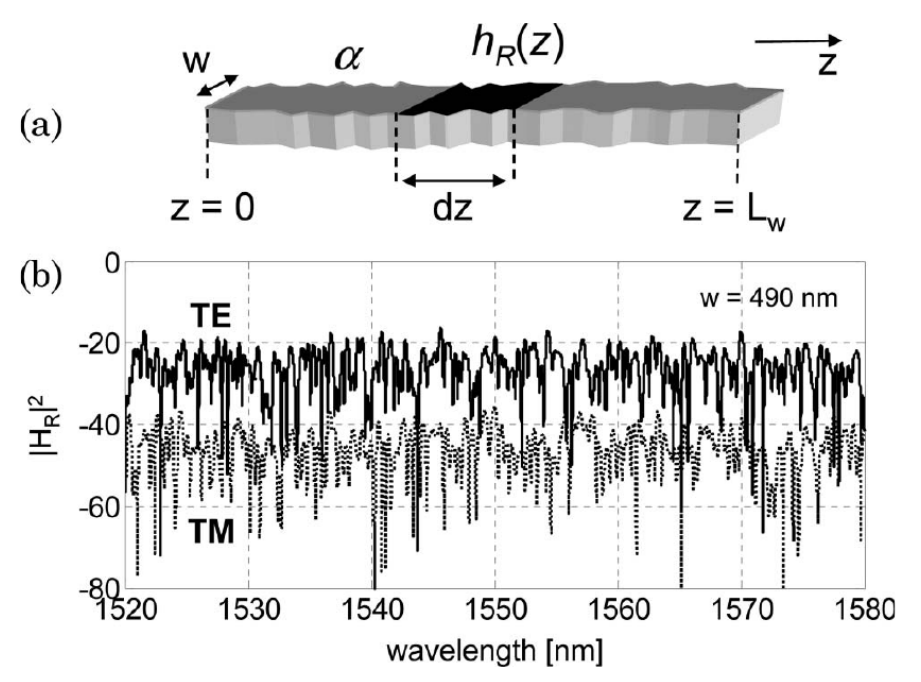

FIGURE 19. (a) Sketch of a waveguide with random sidewall roughness and width $w$, loss coefficient $\alpha$ and length $L_{w}$. (b) Measured power spectral density of the backscattering of a 1-mm-long SOI waveguide with $\mathrm{w}=490$ nm for TE (solid curve) and TM (dotted curve) input polarization. (image reproduced from [127])

An example of measured $\left|H_{R}(\lambda)\right|^{2}$ (normalized to unity input power) for a SOI waveguide [20] with length $1 \mathrm{~mm}$ is shown in Fig. 19(b), for both TE and TM input polarization states. As already shown in Fig. 9 in this type of waveguide the TE mode is much more sensitive to sidewall imperfections and generates a higher backscattering level. The spectral trace of $\left|H_{R}(\lambda)\right|^{2}$ shows the typical wavelength dependence of a white noise, confirming the absence of any dominant deterministic signal component.

The probability density function (PDF) of $H_{R}(\lambda)$ can therefore be estimated, once some considerations on the stationarity and correlation of the the random process have been provided. Rigorously, even if a wide sense stationary (WSS) approximation is assumed for the sidewall roughness (see Sec. 2), backscattering is not a stationary process because the interaction between the propagating mode and the roughness changes with wavelength (because of the mode shape change). However the experimental data reveal that the statistical properties of the backscattering are almost constant over practical bandwidth of tens of nanometers and so a WSS assumption holds well also in this case. Furthermore, the finite length of the waveguide correlates the values of $H_{R}((\lambda)$ at different wavelengths through the convolution with a cardinal sine function which arises from the truncation to a finite waveguide length in the spatial domain. The main lobe half-width $\lambda^{2} / 2 n_{g} L_{w}$ of the function sets the minimum spectral distance to have two uncorrelated wavelengths. Nonetheless we can assume the stationarity of the backscattering in the spectral domain and a correlation which tends to zero increasing the wavelength span, which allow to consider the process as 

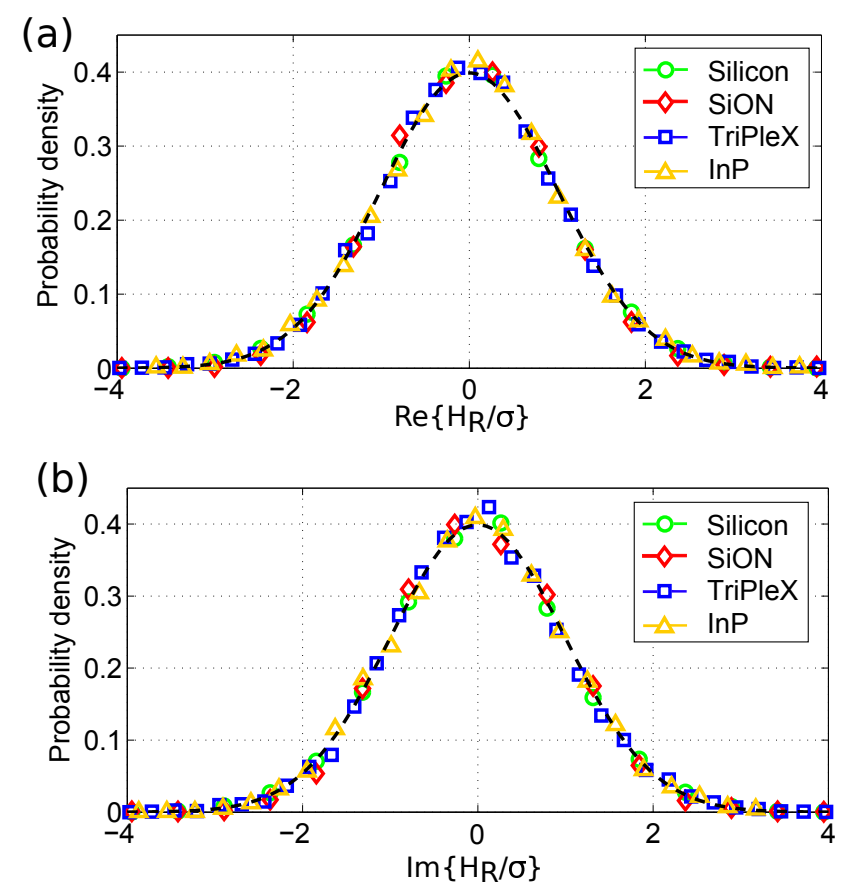

FIGURE 20. Probability density function of the (a) real and (b) imaginary parts of the normalized backscattering for different waveguides: SOI channel waveguide (circles), SiON square buried waveguide (diamonds), box-shaped TriPleX waveguide (squares) and InP rib waveguide (triangles) for TE input polarization. (adapted from [127])

ergodic. In this way a single measure over $\mathrm{N}$ uncorrelated wavelengths carries the same information of $\mathrm{N}$ waveguides measured at single wavelength.

Some measurements of the PDF of $H_{R}(\lambda)$ are shown in Fig. 20 for both real and imaginary parts. Data have been normalized to the standard deviation $\sigma_{R}$ in order to compare the statistics of different technologies. In particular the same waveguides already described in Sec.4.2.a were considered: a SOI channel waveguide, a SiON square buried waveguide, a box-shaped TriPleX waveguide and the InP ridge waveguide in [60], all of them with a width that ensures a single mode propagating regime. Results refer to the TE input polarization but identical behaviour can be observed for TM polarized light. Both the random variables $\mathfrak{R}\left\{H_{R}(\lambda)\right\}$ and $\mathfrak{I}\left\{H_{R}(\lambda)\right\}$ are independently distributed according to a zero-mean normal probability density function (dashed curve), irrespective of shape, size, refractive index contrast, and technology of the waveguide. This statistical behaviour is typical of systems operating in a single scattering regime $[127,128]$. The standard deviation $\sigma_{R}$ of this variables is related to the total backscatter and clearly depends on the particular waveguide. In this case result: $\sigma_{R}^{2}=1.5 \cdot 10^{-3} \mathrm{~mm}^{-1}$ for the SOI waveguide; $\sigma_{R}^{2}=5 \cdot 10^{-6} \mathrm{~mm}^{-1}$ for the SiON waveguide; $\sigma_{R}^{2}=6 \cdot 10^{-6} \mathrm{~mm}^{-1}$ for the TriPleX waveguide; $\sigma_{R}^{2}=2 \cdot 10^{-4} \mathrm{~mm}^{-1}$ 
for the InP waveguide.

The statistical distribution of the real and imaginary part of $H_{R}(\lambda)$ ensures power spectral density $\left|H_{R}(\lambda)\right|^{2}$ being a random variable with a negative exponential distribution, i.e. with the same values for mean and standard deviation. This means that the amplitude of the power oscillations in the wavelength domain increases as the mean backscattering level and, hence, the length of the waveguide. This behaviour is similar to that observed in the forward propagating direction in [124] for the group delay introduced by the waveguide.

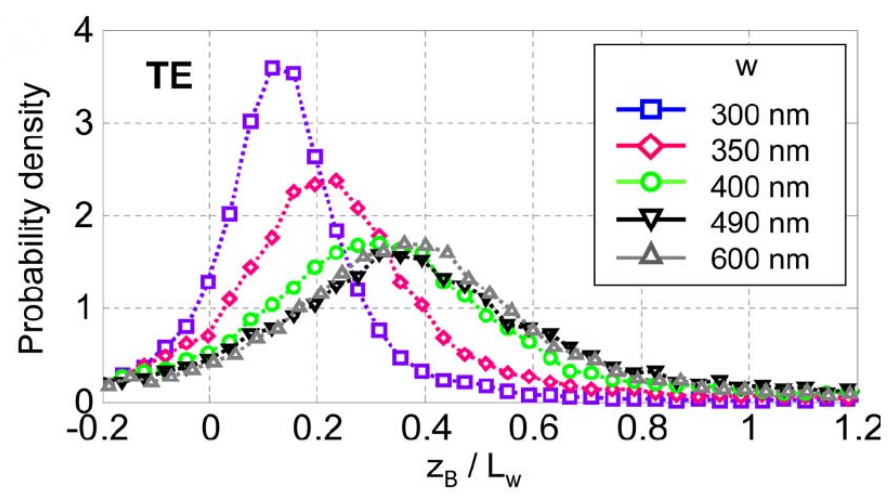

FIGURE 21. Probability density function of the normalized backscattering length of 12-mm-long SOI waveguide for different waveguide width for TE mode. (image reproduced from [127])

The properties of the group delay can be investigated also for the backscattered light in order to understand where actually the power is reflected along the waveguide. Since the phase of $H_{R}(\lambda)$ can be measured, the PDF of the backscattering group length $z_{B}$ can be derived. Figure 21 shows the statistic of the backscattering length $z_{B}$ for the SOI waveguide normalized to the total waveguide length $L_{w}=12 \mathrm{~mm}$ for waveguide widths from $300 \mathrm{~nm}$ to $600 \mathrm{~nm}$ and TE mode. Because of the propagation losses, the maximum of the PDF (average delay/optical length) can be located significantly far from the centre of the waveguide, where it would be expected. It is then possible to define a waveguide effective length

$$
L_{e f f}=\frac{1-e^{-2 \alpha L_{w}}}{2 \alpha}
$$

being $\alpha$ the propagation losses. The average group length is then placed at half of the total waveguide effective length $L_{e f f} / 2$. This implies that in case of low propagation losses the light will be reflected, on average, in the middle of the waveguide $\left(\left\langle z_{B}\right\rangle \simeq L_{w} / 2\right)$ because it has the same probability to be reflected in each point of the waveguide. For the considered SOI waveguide the TE polarization state experiences increasing loss at smaller width (see Fig. 4(b)) and this makes the backscattering being generated mainly in the first part of the waveguide. The tails of the distribution extending also to nonphysical values $\left(z_{B}<0\right.$, 
$\left.z_{B}>L_{w}\right)$ but they are associated to wavelengths in regime of strong attenuation, where the group delay loses the meaning of energy delay and then no violation of the causality principle occurs [129].

As for the models presented in Sec. 4, even the statistical properties of the roughness-induced backscattering can therefore be described in a wide general manner. It acts as a single scattering system, independently of shape, size, refractive index contrast, technology, and state of polarization. The power spectral density of the backscatter follows a negative exponential PDF while the real and imaginary part of $H_{R}(\lambda)$ are independent and identically distributed zero-mean normal random variables. The associated variance is the total backscattered power. Lastly, the light is reflected on average at half of the effective length of the waveguide. As described in the next section, all the analysis performed so far on the nature of the backscattering (from the modelling of the reflected power to its statistical characteristics) can be gathered and exploited to build a realistic models of a waveguide, taking into account also the impact of the sidewall roughness.

\section{Circuital model of the backscattering}

Large efforts have been done in the last decades to propose suitable models for the investigation and simulation of the backscatter. Some of the techniques available in literature have been already presented in Sec. 3, all based on electromagnetic approaches which aim to model the real geometry and the other physical quantities. These approaches can be extremely time and memory consuming, especially for 3-D problems and are rarely useful for the synthesis and analysis of devices and complex circuits that take into account the effect of roughness. For these reasons, in this section a different kind of model is presented, based on a circuit oriented approach. Move from the electromagnetic level to a higher-level circuit abstraction is a well grounded approach in both electronics and microwave fields and can provide fast and very accurate simulation techniques also in photonics [59].

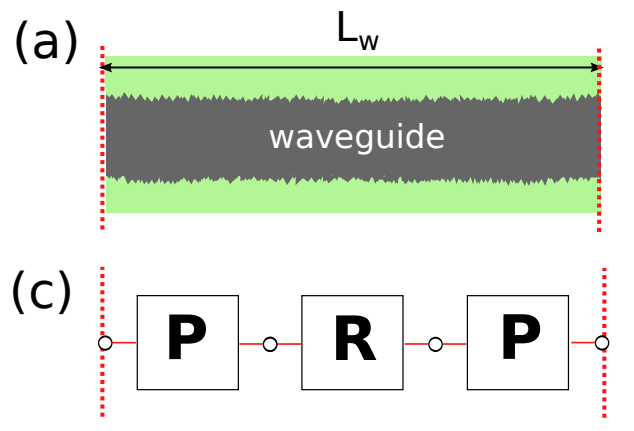

(b)

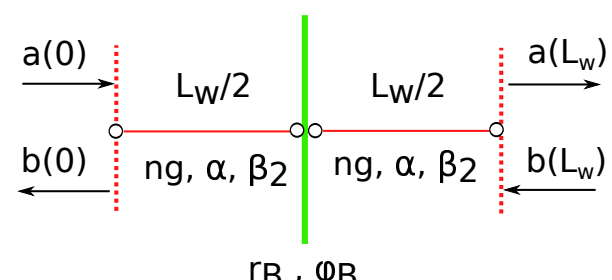

FIGURE 22. (a) Physical structure, (b) equivalent circuit and (c) matrix description of a waveguide with sidewall roughness and length $L_{w} . \mathbf{P}$ is the propagation matrix and $\mathbf{R}$ is the transmission matrix of a random mirror. 
The transfer function of the waveguide with roughness is described as the combination of the pure waveguide propagation (with losses) and the backscatter. The approach used in Sec. 4.1 for the backscattering assumes that the random perturbation of the refractive index profile of the waveguide induced by the sidewall roughness can be described as the superposition of infinite sinusoidal profiles, each one acting as a Bragg grating [20,55]. The equivalent circuit of a grating can be modeled as a partially reflecting mirror with complex reflectivity $r e^{j \varphi}$ placed between two propagating sections of length $L_{w} / 2$, being $L_{w}$ the total physical length of the grating (waveguide), refractive index $n_{\text {eff }}$ and attenuation $\alpha$. In the case of the sidewall roughness of real optical waveguides, each sinusoidal component has an amplitude coefficient which follows a certain probability distribution, generating a random wavelength-dependent reflection (in amplitude and phase) for the central mirror. This probability distribution has been discussed in the previous section with the analysis of the complex function $H_{R}(\lambda)$, derived by Fourier transforming $h_{R}(z)$. Moreover, the analysis of the group delay distribution shows that the power reflection is placed, on average, in the middle of the waveguide, eventually modified by the losses, confirming the intuition based on the Bragg grating analogy.

According to this theoretical description, a suitable equivalent circuit of a waveguide with roughness is shown in Fig. 22(b) where a mirror with random complex reflectivity $r_{B} e^{j \varphi_{B}}$ is placed between two propagating section. This equivalent circuit can then be described by cascading three transmission matrices (see Fig. 22(c)). The first and last sections (matrix $\mathbf{P}$ ) describe the propagation through the half waveguide assumed ideal, without any backscatter. These sections are modeled as

$$
\mathbf{P}=\left[\begin{array}{cc}
e^{-j \theta} & 0 \\
0 & -e^{j \theta}
\end{array}\right]
$$

where $\theta=(\beta(\lambda)-j \alpha) L_{w} / 2, \beta$ is the propagation constant which takes into account the phase effective index $n_{\text {eff }}$, the group index $n_{g}$ and higher dispersion coefficients if necessary and $\alpha$ is the field propagation losses.

The matrix $\mathbf{R}$ provides the transfer function of the central random mirror which takes into account the properties of the backscattered light. As mentioned above, this random reflectivity is described (in the frequency domain) by the complex function $H_{R}(\lambda)$ whose real and imaginary parts are both distributed according to a zero-mean normal probability density function (see previous Sec. 8). The matrix $\mathbf{R}$ is defined as

$$
\mathbf{R}=\frac{j}{T}\left[\begin{array}{cc}
1 & -r_{B} e^{-j \varphi_{B}} \\
r_{B} e^{j \varphi_{B}} & -1
\end{array}\right]
$$

being $H_{R}=r_{B} e^{j \varphi_{B}}$ and $T=\sqrt{1-r_{B}^{2}}$. $H_{R}$ is generated with a Gaussian distribution with variance adjusted to match the desired backscattered power. This can be done defining a random complex reflection in the frequency domain and applying a proper spatial filtering to take into account the finite length of the waveguide. 
Note that the pack scattered power depends only on the variance of $H_{R}$. Finally, the total equivalent transmission matrix of the real waveguide can be calculated as $\mathbf{T}=\mathbf{P} \cdot \mathbf{R} \cdot \mathbf{P}$.
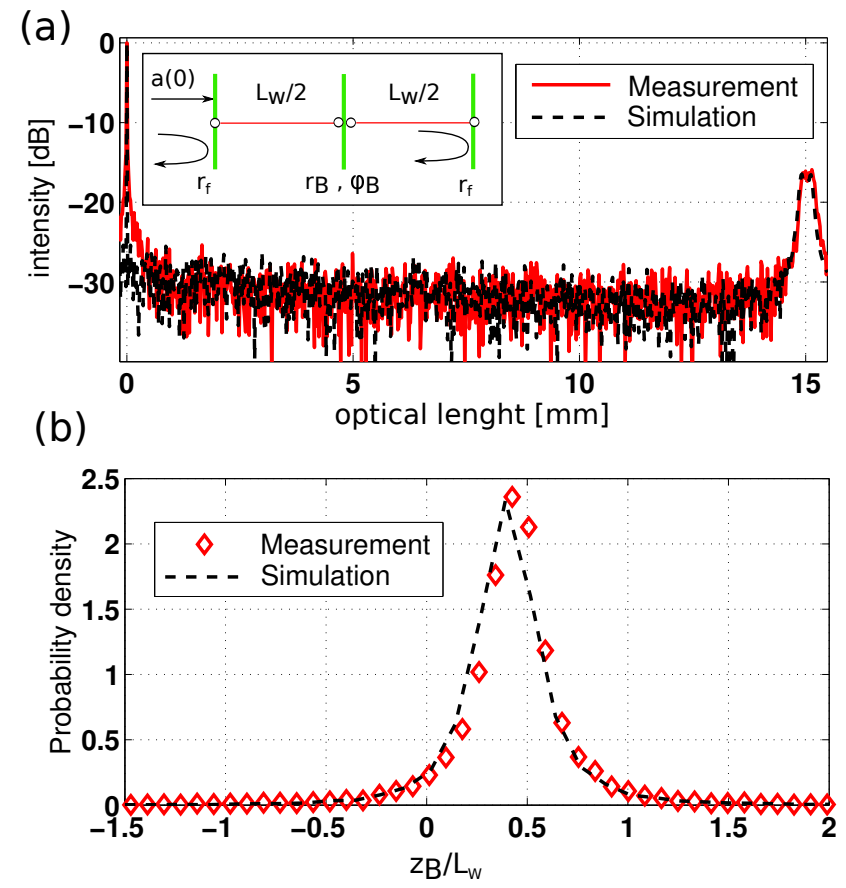

FIGURE 23. Measurement and simulation of a waveguide with sidewall roughness obtained with the proposed circuital model. (a) Spatial distribution of the backscattered power. The effect of distributed backscattering is visible inside the waveguide while the high reflections at zero and $15 \mathrm{~mm}$ are generated by the chip facets. (b) Probability density function of the normalized (distributed) backscattering length (TE mode).

A simple but relevant demonstration of the accuracy of the proposed circuital model is provided by the simulation of the spurious Fabry-Pérot cavity induced by the chip facets on a waveguide with rough sidewalls (see inset Fig. 23(a)). The ridge InP waveguide already considered in the previous Sec. 4 with length $5 \mathrm{~mm}$ (optical group length about $15 \mathrm{~mm}$ ) and width $1.5 \mu \mathrm{m}$ was measured with the interferometric technique mentioned above $[20,57]$ and the spatial (temporal) distribution of the reflections inside the waveguide are obtained by Fourier transforming the spectral response. As can be seen in Fig. 23(a) (red solid line) the distributed backscattering generated by the sidewall roughness is clearly visible as a background-like noise. Two strong reflections are present at the beginning of the waveguide $(\mathrm{z}=0)$ and at $\mathrm{z}=15 \mathrm{~mm}$. They are both generated by the chip facets which act as partially reflecting mirrors.

The simulation was performed adding to the model described in Eqs. (42) and (43) the transmission matrices of the facets (see inset in Fig. 23(a)) for which an internal reflectivity $r_{f}=-30 \mathrm{~dB}$ was considered (facets have an anti-reflective 
coating on the measured sample which guarantees a very small reflectivity). The measured waveguide propagation losses are around $3 \mathrm{~dB} / \mathrm{cm}$ (TE mode). In the model the average backscattered power has been adjusted in order to match the measured value. Results of the simulation, compared to measured data, are shown in Fig. 21(b) (dashed black line) and a very good agreement can be observed between the two responses in terms of backscatter level and variance along the waveguide. The statistic of the backscattering length of both simulated and measured data has been compared as well to have a better evaluation of the accuracy of the model (Fig. 23(b)). As can be seen, simulated data (dashed black line) present the same probability density function of the measured backscattering (red dots), with the same standard deviation and position of the maximum, confirming the correctness of the model also on the group delay distribution. Note that both model and measurements locate the peak at $z / L_{w}<0.5$ because of the losses as well as values outside the range $0<z / L_{w}<1$.

The circuital model of a waveguide with sidewall roughness has been embedded inside a photonic circuit simulator for investigation of the impact of distributed backscattering also within complex optical circuits [130].

\section{Statistical analysis of PhCWs}

Due to the randomness of disorder-induced scattering processes, the study of light propagation in $\mathrm{PhCW}$ s requires some statistical analysis. The main statistical properties of the transmitted and backreflected optical signals are discussed in Sec. 10.1 and Sec. 10.2, respectively, and a comparison with regular optical waveguides is also pointed out. We can anticipate here that a deep change of the statistical properties of $\mathrm{PhCWs}$ is observed when the light propagation undergoes the transition from the dispersive regime (single-scattering) to the diffusive regime (multiple-scattering). Therefore, the statistical description of the light transport in $\mathrm{PhCW}$ s requires that multiple scattering effects are carefully taken into consideration.

\subsection{Statistics of transmission}

A statistical analysis of the light transmission through disordered $\mathrm{PhCWs}$ was carried out by Mazoyer et al. [71] by using a coupled-Bloch-mode formalism, based on a 3D fully vectorial Fourier Bloch-mode method (FBMM), that provides a quantitative prediction of the main statistical transport coefficients. It was pointed out that the transmission probability density function $P(T)$ varies with the group index $n_{g}$ and, in particular, it undergoes a strong change when the light propagation moves for the dispersive (or ballistic) regime to the diffusive regime. The corner point is when the $\mathrm{PhCW}$ length $L$ equals the mean free path length $l_{m}$, this condition roughly corresponding to the end of the ballistictransport regime and to a moderate $50 \%$ attenuation.

Figure 24(a)-(b) shows the simulated transmission density function $P(T)$ of several W1 PHCWs in which the hole radii of the two inner rows are randomly 
(a)

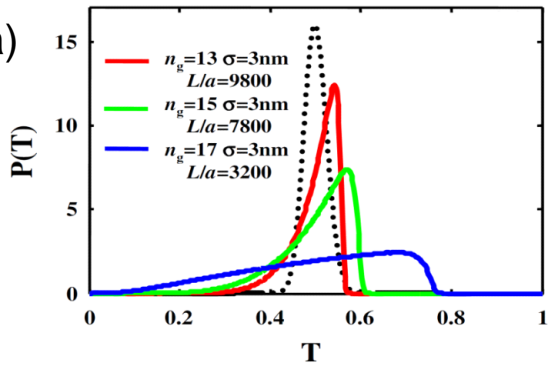

(c)
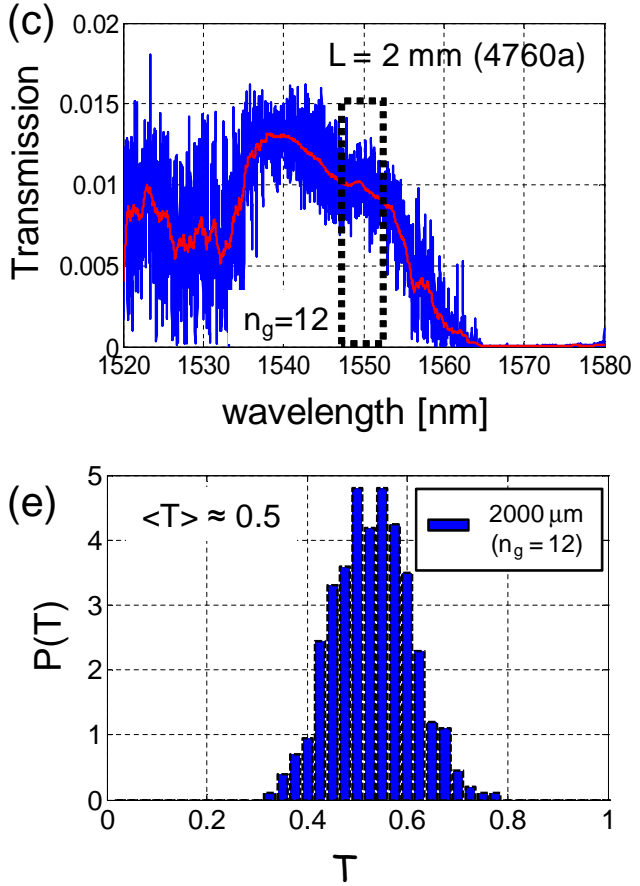

(b)
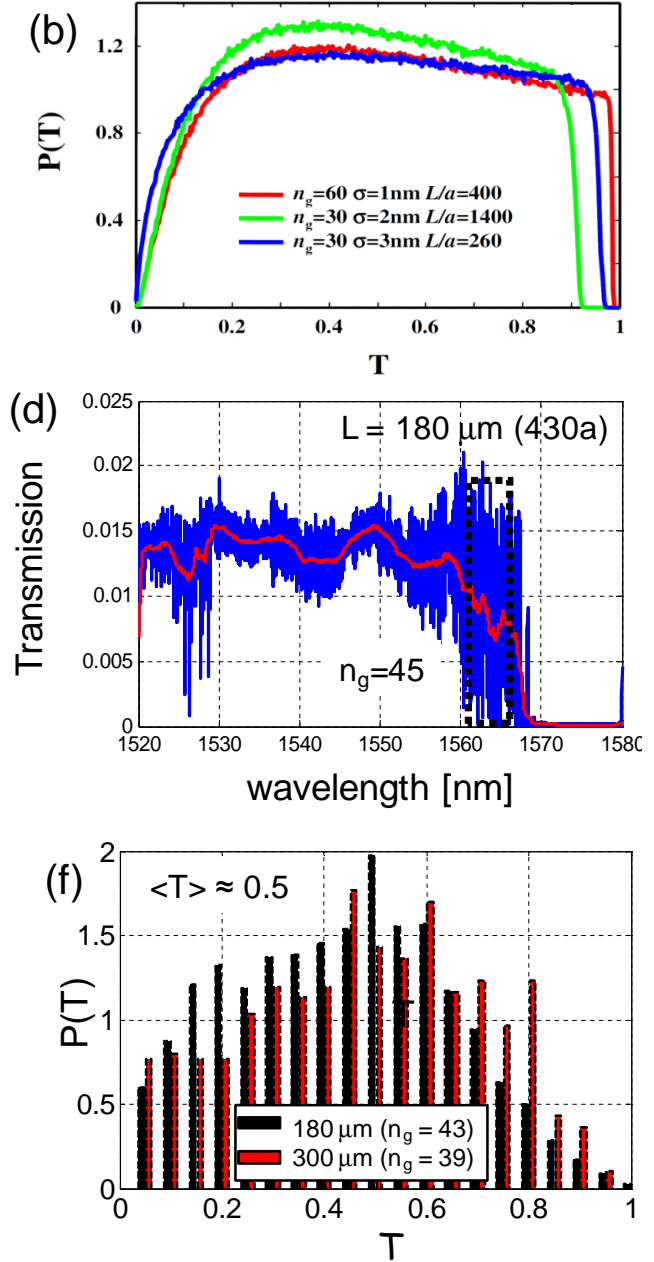

FIGURE 24. Simulated probability density functions $P(T)$ of the light transmitted through W1 PhCWs for (a) a low group index $\left(n_{g}<20\right)$ and (b) a high group index $n_{g}>30$ ) regime. All the waveguides have $\langle T\rangle=0.5$, corresponding to a lengths nearly equal to the effective mean free paths $\left(L \simeq l_{m}\right)$. The dashed curve is (a) is a log-normal distribution with the same standard deviation as the curve obtained for $n_{g}=13$. (c)-(d) Measured transmission spectrum of a suspended membrane dispersion engineered $\mathrm{PhCW}$ s designed according to the scheme of Fig. 11 with a length of (c) $2 \mathrm{~mm}$ and (d) 180 $\mu \mathrm{m}$. Design parameters $\left(a=410 \mathrm{~nm}, r=0.286 a, s_{1}=-48 \mathrm{~nm}, s_{2}=16 \mathrm{~nm}\right)$ are chosen to have an engineered group index of about 40 . Dashed black rectangles mark a 4-nm-wide region where the average transmisison is around 0.5. (e)-(f) Probability density of the transmitted light in the region with $\langle T\rangle$ $=0.5$ for (e) the 2-mm-long PhCWs $\left(n_{g}=12\right)$ shown in (c), and for (f) the $180-\mu$ m-long PhCWs (black bars, $n_{g}=43$ ) shown in (d). Red bars refers to a $300-\mu \mathrm{m}$-long PhCWs with $n_{g}=43$ at $\langle T\rangle=0.5$. ((a) a (b) reproduced from [71]) 
and independently varied around a mean value (0.3a) with a statistical Gaussian distribution of standard deviation $\sigma=3 \mathrm{~nm}$. The length $L$ and the group index $n_{g}$ are chosen in such way that all the waveguides have $\langle T\rangle=0.5$. In the low $n_{g}$ regime [Fig. 24(a)], the shape of $P(T)$ is Gaussian-like with a standard deviation that shrinks as the out-of-plane leakage prevails, i.e., as $n_{g}$ decreases. In the absence of backscattering, the expected probability density is symmetric (dashed curve) [71]. The increasing asymmetry at higher $n_{g}$ indicates the presence of a significant amount of backscattering in the light transport. In the high $n_{g}$ regime, the shape of the transmission probability density radically changes, as shown in Fig. 24(b). Because of multiple scattering effects, $P(T)$ deviates from a Gaussian statistics and resembles a standard uniform distribution. This behaviour is inherently associated with disorder-induced Fabry-Pérot-like resonances along the waveguide, resulting in strong oscillations near the band-edge of the transmission spectrum [see Fig. 17].

The validity of these results is rather general and a similar behaviour is expected in PhCWs with different geometries. For instance, Fig. 24(c)-(d) show the transmission spectra of two PhCWs with a dispersion curve engineered according to the approach depicted in Fig. 11. The structures share the same lattice constant $a=410 \mathrm{~nm}$, hole radius $r=0.286 a$, and hole displacement parameters $s_{1}=-48 \mathrm{~nm}, s_{2}=16 \mathrm{~nm}$. These parameters are chosen in order to have a constant group index of about 40 in the slow-light region between $1560 \mathrm{~nm}$ and $1570 \mathrm{~nm}$. The geometric length of the two waveguides is $L=2 \mathrm{~mm}$ (c) and $L=180 \mu \mathrm{m}(\mathrm{d})$. The dotted rectangle indicates the spectral region where the average transmission $\langle T\rangle$ is around 0.5. For the waveguide of Fig. 24(c) this happens at wavelengths between $1550 \mathrm{~nm}$ and $1554 \mathrm{~nm}$, where the group index is about 12, while for the waveguide of Fig. 24(d) it occurs closer to the band edge (between $1560 \mathrm{~nm}$ and $1564 \mathrm{~nm}$ ), where the group index is about 45 and strong oscillations take place in the transmission spectrum. Figure 24(e) shows the normalized histograms of the intensity transmission of the 2-mm-long waveguide in the wavelength region marked in 24(c). The probability density function $P(T)$ is symmetric and Gaussian-shaped, with a small standard deviation $\sigma_{T}$ of about 0.076. In agreement with theoretical predictions, Fig. 24(f) shows that $P(T)$ dramatically broadens in waveguides with a higher $n_{g}$. Black bars show the normalized histogram of the $180 \mu \mathrm{m}$-long waveguide in the wavelength region marked in 24(d), while the red bars refer to the intensity transmission histogram of a $300 \mu \mathrm{m}$-long waveguide, where $\langle T\rangle=0.5$ at $n_{g}=39$. The standard deviation is found to be $\sigma_{T}=0.22$ for $L=180 \mu \mathrm{m}$ and $\sigma_{T}=0.23$ for $L=300 \mu \mathrm{m}$, that is about three times that one of measured in the low $n_{g}$ regime of Fig. 24(e), and comparable with the standard deviation of 0.288 of a uniform distribution with equidistributed values in the interval $\left[\begin{array}{ll}0 & 1\end{array}\right]$. Similar results were also found on the statistics of transmission though W1 PhCWs [131].

Despite a $3 \mathrm{~dB}$ transmission loss can be accepted in many applications, these results implies that the practical exploitation of $\mathrm{PhCWs}$ strongly depends on the group index at which they operate. In the high index regime, multiple scattering 
makes the actual transmission of a $\mathrm{PhCW}$ be not under control, because there is almost the same probability of getting very high transmittance or unacceptable high transmission losses. This sets severe constraints on the effective use of slowlight optical processing.

\subsection{Statistics of backscattering}

In this section, the statistics of the backscattered light is experimentally investigated with the help of time-frequency reflectance maps (TFRM) obtained by using a coherent OFDR technique [20]. Through the use of TFRMs, the light backscattered by a $\mathrm{PhCW}$ at wavelength ranges of interest can be straigthforwardly isolated and histograms can be numerically built, providing information on the backscattering probability density in different propagation regimes. As in the case of the transmitted light, the statical properties of backscattering are found to dramatically change when the light propagation moves from the dispersive regime to the diffusive regime.

Figures 25(a) and (b) show the TFRM of the $180 \mu \mathrm{m}$-long dispersion engineered waveguide of Fig. 24(d), while Fig. 25(b) shows the TFRM of the 2-mmlong waveguide of Fig. 24(c). The initial point of the horizontal optical length axis corresponds to the input facet of the sample, while the position of the output facet shifts versus wavelength along the optical length axis because of the group index dispersion of the $\mathrm{PhCW}$. Both $\mathrm{PhCWs}$ are enclosed between two input/output ridge waveguides, whose interface with the $\mathrm{PhCW}$ are schematically indicated in the figure. The dotted rectangular regions marked in the top part of the maps indicate the backscattering signal in the wavelength range between $1540 \mathrm{~nm}$ and $1545 \mathrm{~nm}$, where transmission is maximum $(T \simeq 1)$ and the group index is about 7 . Figure 25(c) shows the histogram of the backreflected light intensity $R$ measured in this wavelength range, normalized to the mean reflectivity $\langle R\rangle=0.5$, for both the short waveguide (blue diamonds) and long waveguide (green squares) waveguide of Fig. 25(a) and (b). The intensity probability densities $P(R /\langle R\rangle)$ nicely overlaps to an exponential model (dashed black line), which is a signature of the backscattering statistics of single-scattering systems. The intensity probability density of the light backscattered by a regular silicon waveguide is added for a direct comparison (red triangle). These results confirms that, in the low $n_{g}$ regime, $\mathrm{PhCWs}$ behave as single-scattering systems and can be realistically described by using the circuit model discussed in Sec. 9 for regular waveguides.

The dashed rectangles in the maps of Fig. 25(a) and (b) indicate the backscattering signal in the wavelength range where transmission is around 0.5 . The histograms of backreflected light intensity $R$ measured in these regions are shown in Fig. 25(d). As discussed regarding to the statistics of the transmitted light intensity of Fig. 24, when $T=0.5 \mathrm{PhCW}$ s can operates in very different regimes. The $2 \mathrm{~mm}$-long waveguide, exhibiting a moderately low $n_{g}=12$ in the wavelength range between $1550 \mathrm{~nm}$ and $1554 \mathrm{~nm}$, works in single scattering regime, so that the probability density of backreflection $P(R)$ (white squares) still follows 
(a)

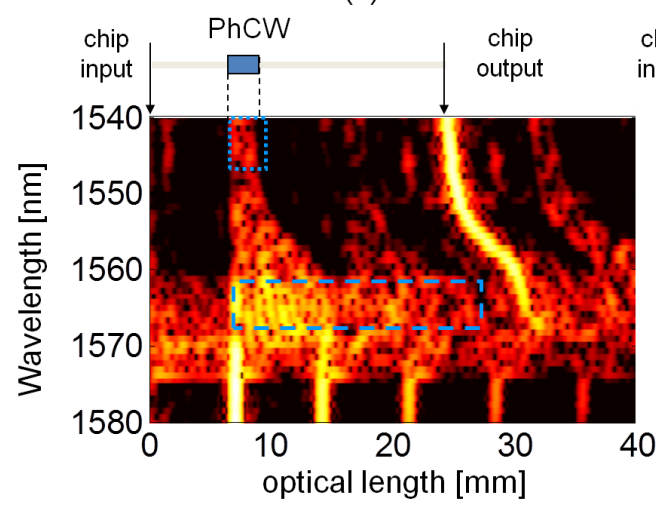

(c)

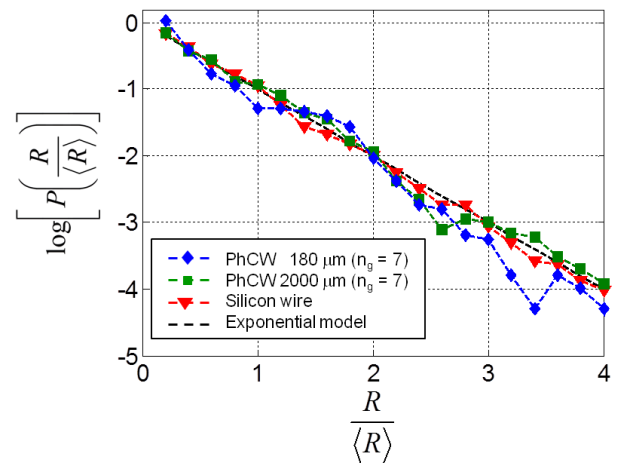

(b)

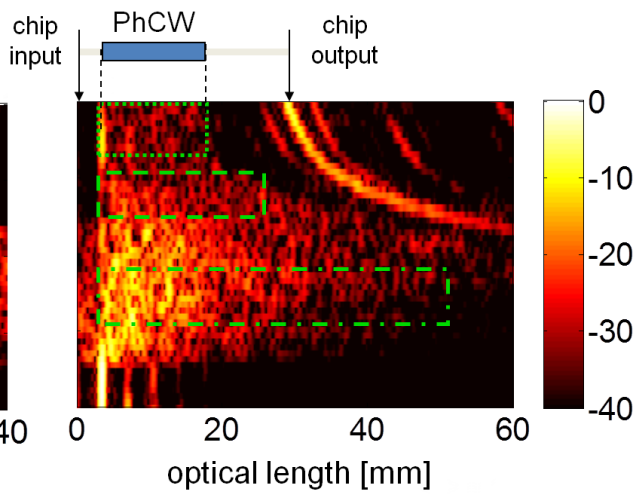

(d)

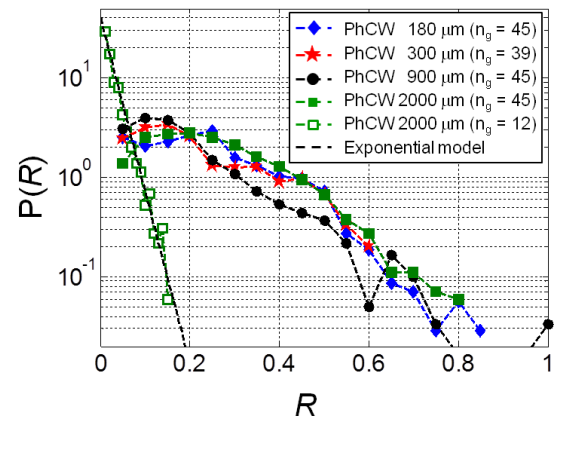

FIGURE 25. Measured TFRM of the dispersion engineered PhCWs of Fig. 25 with a length of (a) $180 \mu \mathrm{m}$ and (b) $2 \mathrm{~mm}$. Dotted rectangles indicate the region of almost unitary transmission $(T \simeq 1)$ and low group index $\left(n_{g}\right.$ $=7$ ). Dashed rectangles mark the wavelength range where $T=0.5$, this condition occurring at $n_{g}=45$ in (a) and at $n_{g}=12$ in (b). The dasheddotted rectangle of (b) shows the region of localized states, where $n_{g}=$ 45, $T<<1$ and the localization length $l$ becomes much smaller than $L$. (c) In the low group index regime $\left(n_{g}=7\right)$ the probability density of the light intensity backscattered by the short PhCW (blue diamods) of (a) and by the long PhCW (green squares) of (b) follows the exponential law (dashed black line) of single scattering systems. Red triangles shows the probability density of the backscattering measured in a 5-mm-long silicon wire. (d) In the high group index regime $\left(n_{g}>40\right)$, multiple scattering makes the the probability density of backscattering strongly deviates from the behaviour of single scattering systems (dashed black line). Measurement of four waveguides with increasing $L$ and operating in the multiple scattering regimes are shown: $180 \mu \mathrm{m}$ (blue diamonds), $300 \mu \mathrm{m}$ (red stars), $900 \mu \mathrm{m}$ (black circles), and $2000 \mu \mathrm{m}$ (green squares). White squares refer to the $2000 \mu \mathrm{m}$-long PhCWs in the low group index regime $\left(n_{g}=12\right)$.

an exponential model (dashed curves), as in the case of Fig. 25(c). Conversely, at wavelengths between $1560 \mathrm{~nm}$ and $1564 \mathrm{~nm}$, the group index of the 180 
$\mu \mathrm{m}$-long waveguide is about 45 and multiple scattering effects arise, which are clearly visible in the TFRM of Fig. 25(a) at an optical length between $8 \mathrm{~mm}$ and $12 \mathrm{~mm}$. As shown in Fig. 25(d), the presence of multiple scattering makes the probability density of backreflection of the $180 \mu \mathrm{m}$-long waveguide (blue diamonds) strongly deviate from the exponential law. This change in the shape of $P(R)$ nicely agrees with the theoretical predictions on the coherent backscattering statistics in disordered random media [132]. A similar behaviour was also observed in the probability density of the $2000 \mu \mathrm{m}$-long waveguides (green squares) in the region of $n_{g}=40$ [dashed dot rectangle in Fig. 25(b), $1560 \mathrm{~nm}$ $<\lambda<1564 \mathrm{~nm}$ ], and in waveguides with $L=300 \mu \mathrm{m}$ (red stars) and $900 \mu \mathrm{m}$ (black circles) with group index in the order of 40 .

A further consideration can be done about the occurrence of localized sates. By applying the definition of localization length [133],

$$
l=-\frac{L}{\langle\ln T\rangle},
$$

it is found that, in the wavelength range considered in Fig. 25(d), the localization length of the waveguides with length $L=900 \mu \mathrm{m}$ and $2 \mathrm{~mm}$ is about $150 \mu \mathrm{m}$ and $300 \mu \mathrm{m}$, respectively, that is much less than $L$. Even though the condition for strong localization $(k l<1)$ is not rigorously fulfilled [133], when $l<<L$ localized photonic states are expected to be originated into the waveguide [134-136]. Therefore, the backreflection probability density of Fig. 25(d) applies also to $\mathrm{PhCWs}$ where multiple scattering originates localization phenomena.

A strong change in the probability density from the dispersive to the diffusive regime was also observed in the statistics of the out-of-plane scattered light intensity by Garcia et al. [134].

These results demonstrate that in the multiple scattering regime the behaviour of $\mathrm{PhCWs}$ strongly deviates from that of classical waveguides. However, once the statistics of the transmitted, back-reflected, and radiated light in the high $n_{g}$ regime are known from either EM simulations or experiments, a circuit model can be built (as in the case of the low $n_{g}$ regime) by following the approach described in Sec. 9. Since the statistical properties fully characterize the $\mathrm{PhCW}$ response, multiple scattering effects and the occurrence of localized states are inherently taken into consideration in the circuit model.

\section{Impact of the waveguide backscatter on circuits}

Waveguide roughness can be responsible of severe degradation of the performances of an optical integrated circuit. Counter propagating power not only can sums up along the waveguides resulting in very high, wavelength dependent, reflections as shown in Tab. 2, but also generate spurious responses, ghost images, instabilities, and so on. Further, active components are generally the most sensitive devices to backreflections since they can generate unwanted lasing effects, nonlinear amplification, resonant spectral reponses or deviation from 
the optimum working point. Despite the large amount of studies devoted to the analysis of the impact of lumped reflections on these devices, investigation of distributed effects has not attracted the same attention to date.
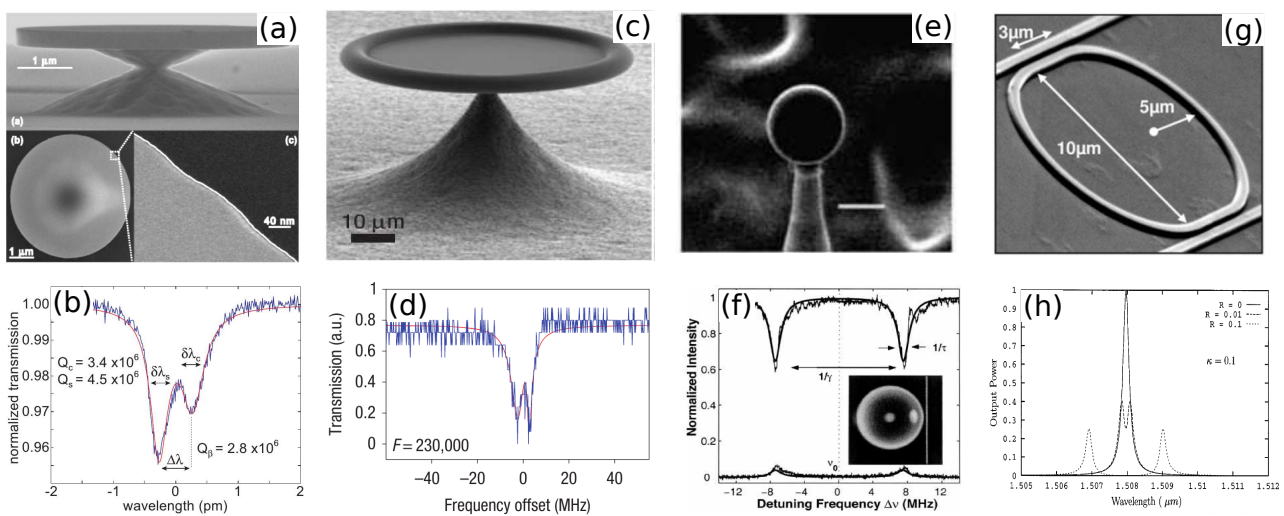

FIGURE 26. Effect of the sidewall roughness on different types of optical resonators. (a) Disc (adapted from [137]; (b) doublet mode resonance for disc of radius $30 \mu \mathrm{m}$ (adapted from [138]; (c) toroid (adapted from [139]); (d) transmission spectrum of a toroidal resonator showing optical modesplitting (adapted from [140]); (e) sphere (adapted from [141]); (f) spectral transmission and reflection of a 70- $\mu m$ sphere (reproduced from [142]; (g) ring (adapted from [143]); (h) response of a ring resonator filter for different values of backscattering (reproduced from [144])

On the contrary, the effect of backscatter have been widely investigated in literature on passive devices and in particular on resonant circuits, whose transfer function can largely enhance even very small reflections. Micro-discs, [137,145], toroids [140], spheres [146] and micro-rings [90,144] are all travelling-wave optical resonators which show characteristic behaviours in presence of counterpropagating light, as those shown in Fig. 26. In all these structures each propagating mode is naturally two-times degenerate since two possible propagating directions exist (clockwise and counter-clockwise) for each of them. When part of the power is exchanged between this two degenerate modes via a coupling mechanism (backsattering) a splitting of the degeneracy can be observed, as always happen in physics in presence of coupling $[142,147]$. Backscattering breaks the unidirectional light propagation and as consequence two resonant frequencies appear symmetrically located with respect to the natural resonant point, degrading the overall quality of the resonance, that is broadening the notch width. This effect can be observed on all the aforementioned resonators as shown in Fig. 26(b, d, f).

Another relevant effect that can be observed in presence of backscattering for cavities working near the resonance is that the cavity operates as a narrow-band mirror. Let's consider the case of an SOI ring resonator all-pass filter (i.e. a ring coupled to a single bus waveguide) with sidewall roughness, as shown in Fig. 
27(a). $K$ is the coupling coefficient, $L_{r}$ the ring length and $h_{r}$ and $h_{t}$ the reflected and transmitted time-domain signals, respectively. $r_{e}$ refers to the reflection of the output chip facet and the ring radius is $20 \mu \mathrm{m}$ [90].

(a)

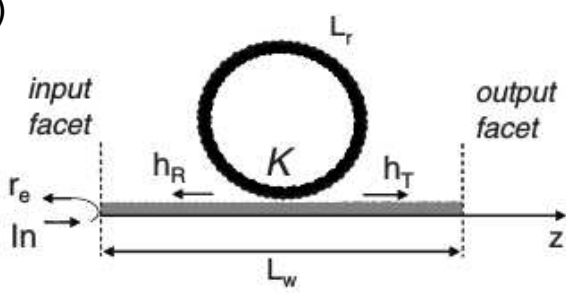

(c)

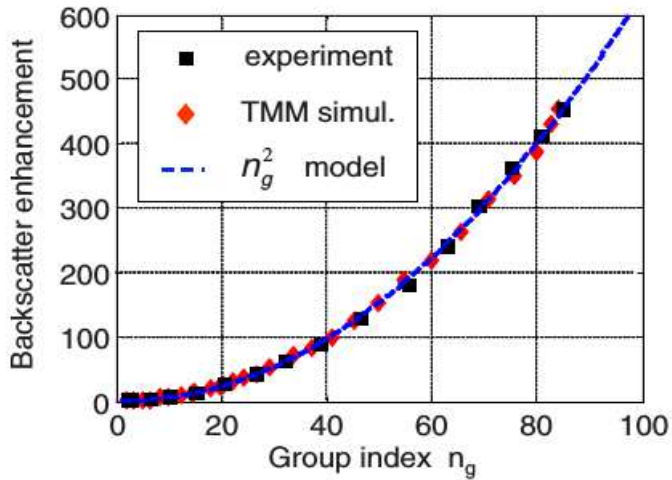

(b)
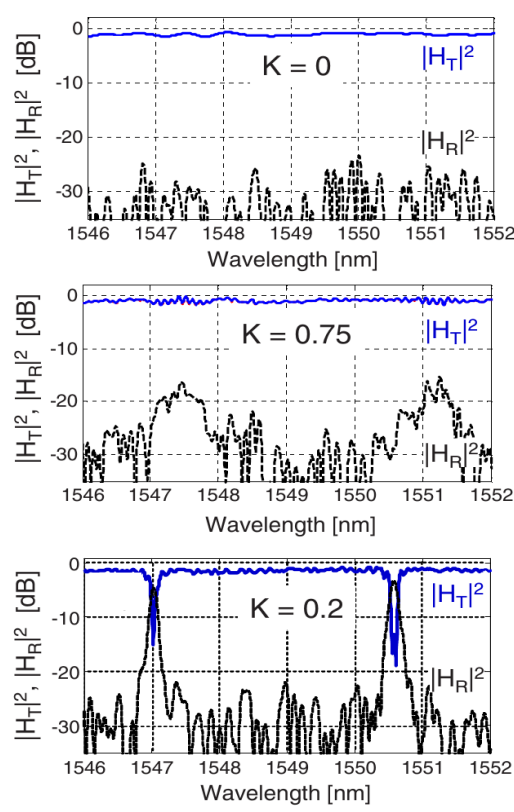

FIGURE 27. (a) Schematic of an integrated ring resonator with waveguides with roughness on both bus and ring; (b) measured transmission $\left(\left|H_{T}\right|^{2}\right)$ and backreflection $\left(\left|H_{R}\right|^{2}\right)$ for different coupling coefficients; (c) backscattering enhancement at resonance due to the ring resonator as function of the group index: (black squares) experiment, (red diamonds) TMM simulations, and (blue dashed curve) numerical fit with a $n_{g}^{2}$ model (adapted from [90]).

Both the transmitted and reflected spectra $\left|H_{T}\right|^{2}$ and $\left|H_{R}\right|^{2}$ were measured for different power coupling coefficients $K$ as shown in Figs. 27(b). $K=0$ refers to the $3 \mathrm{~mm}$-long isolated bus waveguide since no coupling with the cavity occurs. In this case the backscattering level (black curve) is more than $30 \mathrm{~dB}$ lower than the transmission, resulting in $-35 \mathrm{~dB} / \mathrm{mm}$ average backscattering. When $K=0.75$ the resonator is working in a highly overcoupled regime: as expected no resonances are observed on the transmitted power and the reflected signal shows just a small increasing as effect of the cavity. When the coupling decreases to $K=0.2$, light resonance induces sharp transmission notches. At these wavelengths, however, the round-trip losses cannot completely explain the notches' depth as most of the light is scattered back to the input port resulting to a reflection level even higher than the ring transmission. For example, at a wavelength of $1550.5 \mathrm{~nm}$ transmitted power is less than $-15 \mathrm{~dB}$ while reflection is around $-5 \mathrm{~dB}$ with respect to the input. At different resonant wavelengths transmission and backscatter are different because of the random nature of the reflecting 
mechanism. It is also interesting to observe how the multiple round trips inside the ring strongly correlate the backscatter which does not exhibit a white-noiselike power spectral density as in isolated waveguides (e.g. the case $K=0$ or Fig. 19). Similar effects have been observed also at the ideally-isolated add port of add-drop ring filters [148].

In [90], for all the cases shown in Fig. 27(b), a corresponding group index of the resonator was also measured as $n_{g}=\tau_{g} L_{r}$, being $\tau_{g}$ the group delay of the ring. Since approaching the resonance the group delay increases accordingly (because of the light trapping effect), a measurement of the dependence of the backscattering on this parameter was performed. The cavity-enhanced backscattering (ratio between the actual backsattering and that generated by a straight waveguide) was found to scale up according to the square of the group index. In Fig. 27(c) experimental data (black dots) are compared to transfer matrix simulation (red dots) and fitted with $n_{g}^{2}$. The origin of this dependence is related to the inherent periodicity of the ring that allows the backscatter to coherently sum up at each roundtrip with a phase-matched mechanism [144]. It is then intrinsically different from the dependence observed in Sec. 4, where the backscattered power has been related to the quantity $\left(n_{g}-n_{e f f}\right)^{2}$. In this case the group index changes because it is "circuit related"rather than "waveguide related". The backscatter scales linearly with $L_{r}$ and for a given product $n_{g} L_{r}$, large rings with small $n_{g}$ have less backscattering than small rings with high group index [90]. Further considerations related to the use of ring resonators as delay lines are discussed in [149].
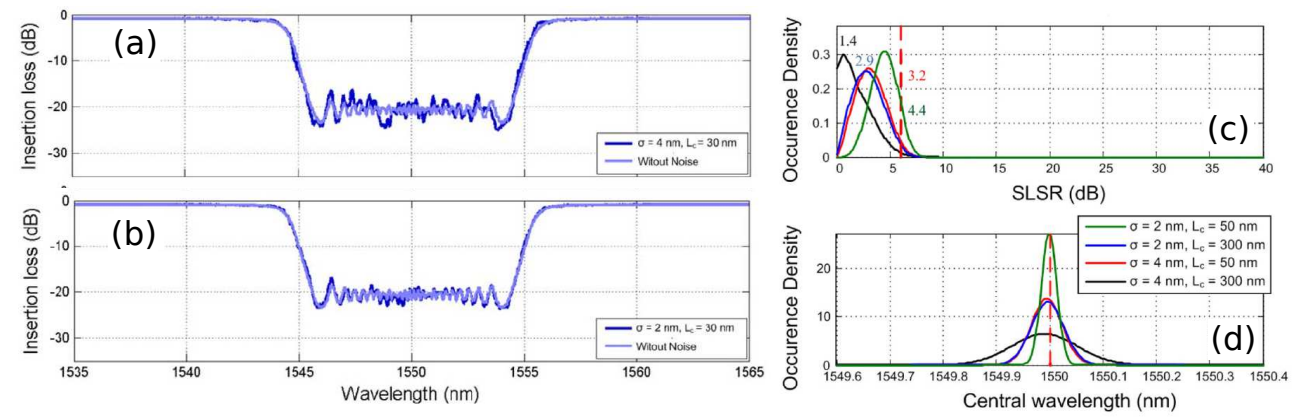

FIGURE 28. Simulation of a chirped Bragg Grating with sidewall roughness standard deviation of $2 \mathrm{~nm}$ and length of (a) $300 \mathrm{~nm}$ (b) $30 \mathrm{~nm}$. (c) and (d) show, respectively, the distribution of the side lobe suppression ratio (the labels are the average SLSRs) and the central wavelength of a uniform grating for different roughness parameters (adapted from [150]). The dotted lines represent the results in the ideal case.

Another integrated device which can suffer heavy impairments related to sidewall roughness is the Bragg grating. Performances of long gratings can be severely degraded, in particular on high-contrast waveguides such as SOI, resulting in distortions of the spectral response. An example of these effects has been 
shown in [150] exploiting an ad-hoc simulator and is reported in Fig. 28. The effect of the roughness standard deviation $\sigma$ on the transmission of a first order grating of length $2.8 \mathrm{~mm}$ and chirp rate of $14 \mathrm{~nm} / \mathrm{cm}$ is shown in Fig. 28(a) for a roughness correlation length of $30 \mathrm{~nm}$. Even with $\sigma=4 \mathrm{~nm}$, a reasonable value for most of the technological processes (see Tab. 1), the in-band behaviour of the grating shows a significant deviation from the ideal characteristic. It is also interesting to observe the results of a statistical investigation on the performances of a set of gratings in terms of Side Lobe Suppression Ratio (SLSR) and central wavelength when random sidewall roughness is taken into account. Figure 28 (b,c) show the Gaussian fits of the occurrence densities related to four sets of 1000 uniform gratings which differ for the roughness parameters $(\sigma=2,4 \mathrm{~nm}$, $\left.L_{c}=50,300 \mathrm{~nm}\right)$. The dotted lines represent the result of an ideal uniform grating. As can be seen backscattering has a big impact on the SLSR and in the worst case $\left(\sigma=4 \mathrm{~nm}, L_{c}=300 \mathrm{~nm}\right)$ only $10 \%$ of the devices shown a SLSR $>3 \mathrm{~dB}$ while for $\sigma=4 \mathrm{~nm}, L_{c}=50 \mathrm{~nm}$ (reasonable case) this number grows to $55 \%$. In the best case $\left(\sigma=2 \mathrm{~nm}, L_{c}=50 \mathrm{~nm}\right) 30 \%$ of the devices presents a SLSR $>5 \mathrm{~dB}$. Backscattering affects the central wavelength as well, not in terms of average value (which is exactly what expected in the ideal case) but as a broadening of the probability density. These results suggest that the effect of sidewall roughness must be considered during the device design in order to maximize the yield of the production process.

\section{PhCWs and coupled resonator optical waveguides (CROWs)}

The statistical analysis presented in Sec. 10 demonstrates that $\mathrm{PhCWs}$ in the low $n_{g}$ regime behave as classical waveguides; conversely, in the high $n_{g}$ regime, multiple scattering and localization effects arise which have no counterpart in classical waveguides. Therefore, one could wonders if a classical waveguide structure equivalent to a $\mathrm{PhCW}$ in the high $n_{g}$ regime does exist.

An example, it can be found in the concept of the so called coupled resonator optical waveguides (CROWs) [151, 152]. These structures, consisting of a sequence of directly coupled resonators, have been realized by cascading Fabry-Pérot cavities [153], microring resonators [154], microtoroids [155], microspheres [156], cuboid microresonators [157], and photonic crystal cavities [158].

Similarly to PhCWs, CROWs can support slow light propagation. The light can propagate through a CROW only within a discrete number of transmission passbands, where the group velocity is reduced according to a slowdown factor $S$ that scales linearly with the finesse of the resonators [159]. A strict analogy exists between the group index of CROWs (which is proportional to $S$ ) and the group index of PhCWs. Fig. 29(a) shows the measured $n_{g}$ versus wavelength of the W1 PhCW of Fig. 13, while Fig. 29(b) shows the simulated $S$ of a CROW with a bandwidth $B$ centered around the wavelength $\lambda_{0}$. The simulated CROW is made of 50 ring resonators with $9 \%$ power coupling coefficients in the central section

of the structure, while the coupling coefficients at the input/output sections of 

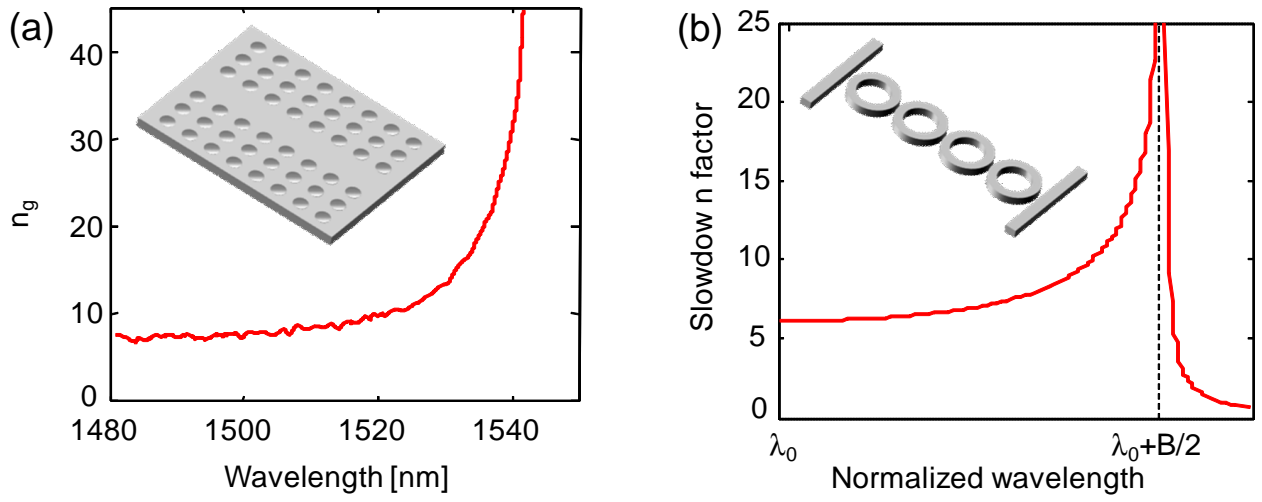

FIGURE 29. Comparison between the group index of PhCWs and CROWs: (a) measured group index versus wavelength of the W1 PhCW of Fig. 13;

(b) simulation of the typical frequency domain behaviour of the slowdown $S$ of a CROW, corresponding to the enhancement factor of the CROW group index with respect to the group index of the waveguide realizing the CROW.

the rings' chain are apodized according to the rules given in Ref. [160]. Due to symmetry of the CROW spectrum with respect to the central wavelength $\lambda_{0}$, the $S$ curve is shown only for wavelengths larger than $\lambda_{0}$. The $S$ curve strongly resembles the $n_{g}$ curve of the $\mathrm{PhCW}$, both exhibiting a steep increase as the wavelength approaches the band edge. This similarity implies that, despite the evident differences between these structures, under certain operative conditions $\mathrm{PhCWs}$ and CROWs can indeed share more features than expected, exhibiting for instance similar propagation loss, slowdown factor (i.e. $n_{g}$ ), delay tunability, footprint and ultimately sensitivity to disorder [149]. However, it should be noticed that within the CROW bandwidth (extending typically across a few tens or hundreds of $\mathrm{GHz}$ ) the waveguide mode can be assumed unaltered, while in the case of $\mathrm{PhCWs}$ the $n_{g}$ increase is typically associated with a significant reshaping of the Bloch mode (see Sec. 6.3).

In CROWs disorder effects arise from tolerances in the coupling coefficients between resonators (coupling disorder) [161], misalignments of the resonant frequencies due to random variations of the resonators' optical lengths (phase disorder) [162] and sidewall roughness [20]. All these sources of disorder produce similar effects on the CROW response, because they break the periodicity of the structure, acting as partial reflectors, and contribute to the backreflection of the device.

A comprehensive study of disorder effects in CROWs was theoretically and experimentally carried out in the work by Ferrari et al. [162], where the contribution given by coupling and phase disorder effects were isolated and individually studied, in order to reach a deeper comprehension of the mechanism relating the nature and strength of disorder to the optical response of CROW devices. 


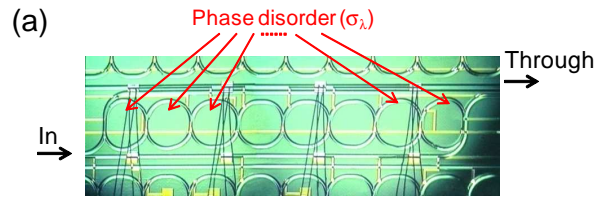

(c)
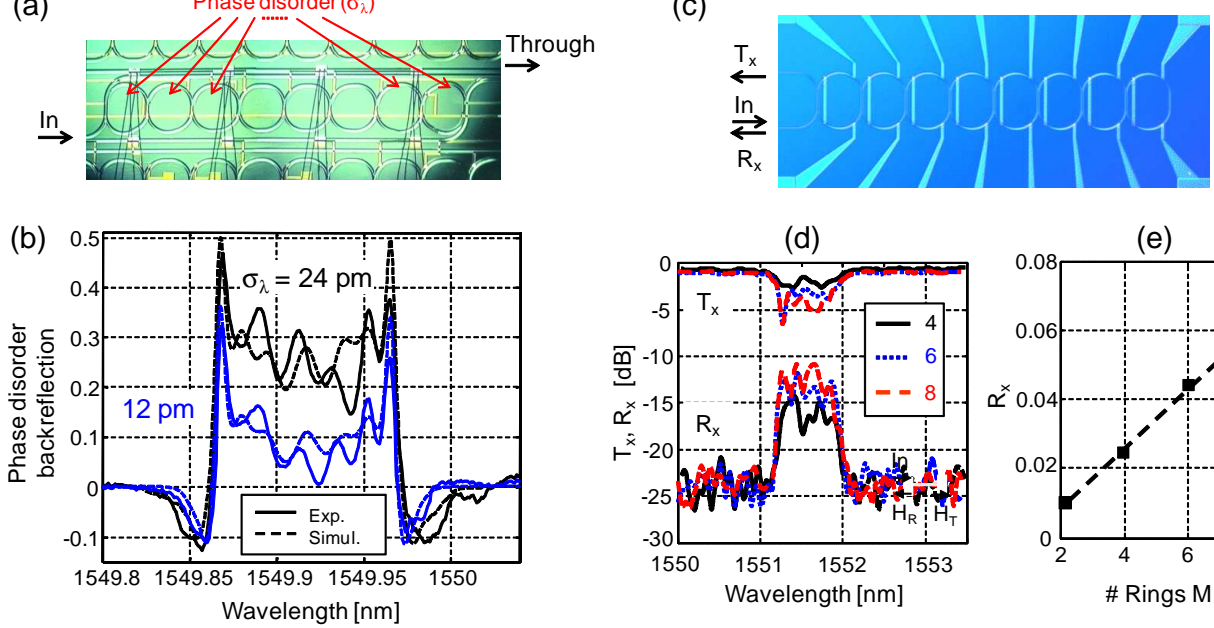

(d)

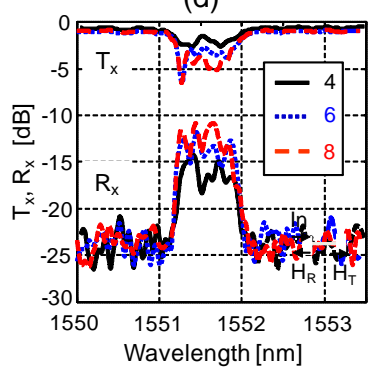

(e)

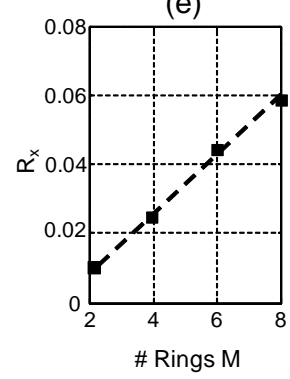

FIGURE 30. (a) Top view photograph of an 8-ring CROW with a metal heater on each ring to independently control the resonance frequency of each resonator and set an arbitrary degree of phase disorder. The device is fabricated in $4.5 \%$ index contrast SiON technology. (b) Average backreflection of the eight-ring CROW for a phase disorder standard deviation $\sigma_{\lambda}=$ $12 \mathrm{pm}$ (solid blue line) and $24 \mathrm{pm}$ (solid black line). Dashed curves show the result of numerical simulations performed through transfer matrix method. Images in (a) and (b) are adapted from [162]. (c) Top view photograph of a tunable 8-ring CROW realized in SOI technology. (d) Measured Through port transmission $\left(T_{x}\right)$ and In port backreflection of SOI CROWs with $M$ $=4$ (red solid curves), 6 (blue dotted curves), and 8 (black dashed curve) coupled RRs. (e) In-band average backreflected power of SOI CROWs vs the number $M$ of coupled RRs. Images (d) and (e) are adapted from [90].

To this aim, the structure of Fig. 30(a) was employed, consisting of a tunable ring-resonator CROW where the cavity round-trip phase of each resonator is individually controlled by means of thermo-optic actuators. In this way an arbitrary degree of phase disorder was dynamically introduced without altering the power coupling coefficients between the rings, that is without changing the level of coupling disorder. The ring-based architecture enables also to separate the back-reflection due to phase disorder (outgoing from Through port) from the backscatter due to waveguide roughness (outgoing form the In port), because the two contributions are spatially decoupled at the CROW output waveguides.

Results demonstrate that, similarly to PhCWs, backreflection in CROWs increases with the square of the group index (that is $S^{2}$ ) and with the square of the degree of disorder. Fig. 30(b) shows the measured spectrum of the backreflection caused by phase disorder at the Through port of the CROW of Fig. 30(a). The transmission band of the CROW is $100 \mathrm{pm}$ wide and is centred around a wavelength $\lambda_{0}=1549.915 \mathrm{~nm}$. In this experiment the resonance wavelength of the rings were randomly and independently shifted according to a 
normal distribution with a standard deviations $\sigma_{\lambda}=12 \mathrm{pm}$ (blue solid curve) and $24 \mathrm{pm}$ (black solid curve) and the back-reflected power was averaged over a set of 50 independent disordered configurations. Experimental data are in very good agreement with the results of numerical simulations (dashed lines) all over the measured wavelength range, even at the band edge and in the stop band. The $n_{g}^{2}$ dependence of the backreflection originates the reflections peaks at the band edges, where the group velocity is minimum. The effect is more evident at lower disorder degree $\left(\sigma_{\lambda}=12 \mathrm{pm}\right)$ because when the resonance spread of the resonators increases, the slowdown factor itself is modified by disorder, decreasing significantly at the band edges, while remaining almost unchanged at $\lambda_{0}$ [161]. Around $\lambda_{0}$ the backreflections increases by a factor 4 when the disorder doubles, thus confirming also the dependence on the square of the disorder degree. The negative value of the backreflections means that, in case of disorder, transmission can occur also at wavelengths lying in the stop-band of the unperturbed CROW, producing a broadening of the bandwidth. A similar effect occurs also in PhCWs, where disorder can be responsible for the creation of band tails and, consequently, for a reduction of the band gap width [163]. In the time domain, these distributed backreflections can give rise to detrimental effects on pulse propagation, causing for instance delay fluctuations and envelope distortions [162].

Backreflections due to the roughness induced backscattering of the CROW waveguide can be isolated from the coupling and phase disorder effects because the backscattered light outgoes from the In port of the CROW. An experiment aiming at quantifying the backscattering in CROWs was performed on devices realized on a SOI platform, as the one shown in Fig. 30(c) [90]. The waveguide and the bending radius of the rings are the same as for the device of Fig. 27. Fig. 30(d) shows the Through port transmission $\left(T_{x}\right)$ and the $I n$ port reflection $\left(R_{x}\right)$ of CROWs made of $M=4$ (black solid curves), 6 (blue dotted curves), and 8 (red dashed curve) rings, respectively. The transmission band of the CROWs is about $0.75 \mathrm{~nm}$ and thermal tuning was used to make all the rings resonate at the same wavelength of $1551.6 \mathrm{~nm}$. Results show that the in-band backreflection increases with the number or rings, while the out-of-band backreflection, which is almost entirely due to the bus waveguide alone, is independent of $M$. As shown in Fig. $30(\mathrm{e})$, the average in-band reflection, averaged over the $90 \mathrm{GHz}$ bandwidth of the CROW, increases linearly with $M$. This additive intensity law demonstrates that the power backscattered by each ring (scaling with the square of the ring's group index, as shown in Sec. 11) is uncorrelated with the backscattering of the other rings.

Approaching the edge of the transmission band, the group velocity of CROWs steeply decreases and ideally goes to zero. Yet, as in the case of $\mathrm{PhCWs}$, the maximum achievable slow down of the light is limited by disorder effects. As observed by Mookherjea et al., this limitation is inherently associated to disorder induced localization phenomena arising at wavelengths near the CROW band edge [157]. These effects were studied on the SOI CROW of Fig. 31(a), where a 
(a)

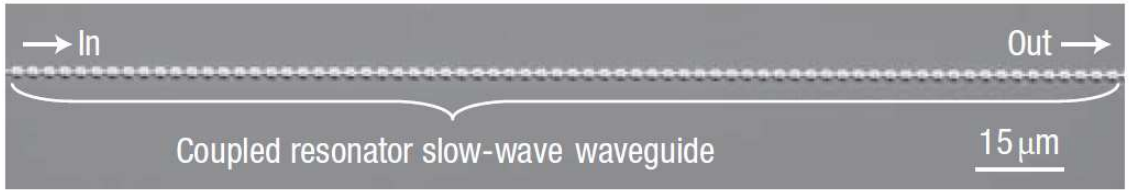

(b)

(c)

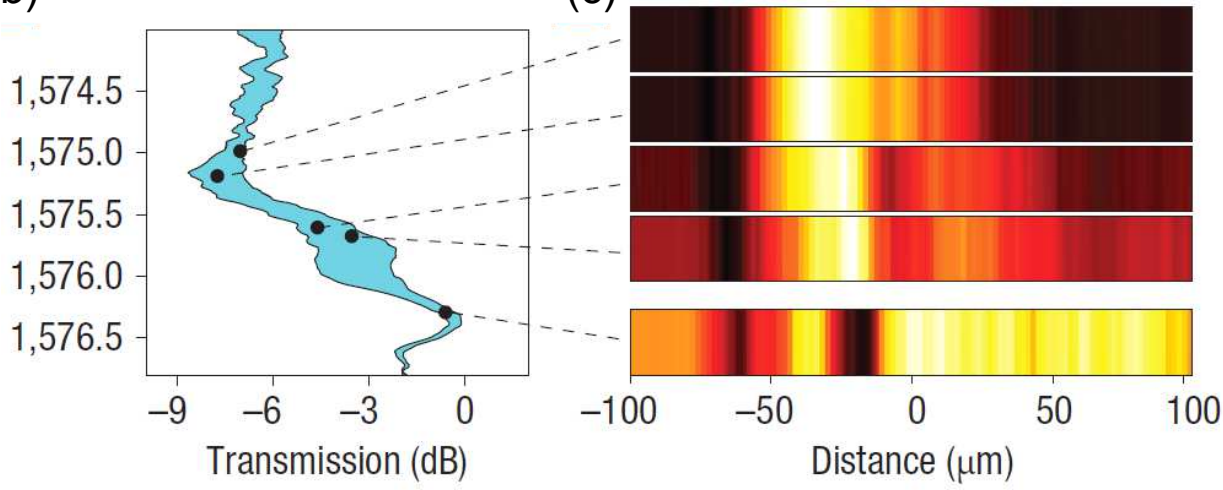

FIGURE 31. (a) Photograph of a SOI CROW consisting of 100 cuboid resonators realized along a silicon nanowaveguide. (b) Measured transmission of the CROW around the edge of a transmission band. The nominal transmission band is located at wavelengths above $1576 \mathrm{~nm}$. (c) Measured profile of the optical field along the CROW at several wavelengths around the band edge: an extended field distribution is observed at $\lambda>1576 \mathrm{~nm}$ (lower $n_{g}$ ), localized field distributions are observed at $\lambda<1576 \mathrm{~nm}$ (higher $n_{g}$ ). (Images adapted adapted from [157]).

single-mode silicon waveguide $(250 \mathrm{~nm}$ width $\times 500 \mathrm{~nm}$ height $)$ was periodically loaded with 100 cuboid resonators $(1.5 \mu \mathrm{m}$ width $\times 1.5 \mu \mathrm{m}$ length $\times 500 \mathrm{~nm}$ height) with a longitudinal periodicity of $2.75 \mu \mathrm{m}$. Figures $31(\mathrm{~b})$ and (c) show the measured transmission and spatial distribution of the optical field along the CROW at wavelengths near the band edge (located around a wavelength of $1576 \mathrm{~nm}$ ). The field profile was measured by using a modified knife-edge method [164]. Results show that the CROW exhibits extended non-localized field distributions in the higher-wavelength region (low $n_{g}$ region, $\lambda>1576.00$ $\mathrm{nm}$ ), while localized field distributions are observed in the lower-wavelength (high $n_{g}$ region, $\lambda<1576.00 \mathrm{~nm}$ ) regions.

A further confirmation of the strict relationship between localization effects in CROW and high $n_{g}$ was also provided by Cooper et al [165]. In this experiment it was demonstrated that localization-free propagation can occur in very long chains $(>100)$ of coupled ring resonators, provided that the structure has a very low slowdown factor $(S \approx 2)$. At higher $S$, phase disorder due to fabrication tolerances in the resonance frequencies of the rings becomes the dominant disorder effect $[162,166]$. Therefore, to increase the maximum achievable $n_{g}$, phase disor- 
der can be compensated through an active control of the rings' resonances, by exploiting for instance local heating of the waveguide [166]. Recently, several setand-forget post-fabrication trimming techniques have been also proposed that enables a permanent correction of effective index of the waveguides [167-174], thereby enabling the exploitation of CROWs at higher $n_{g}$ regimes.

\section{Discussion and conclusion}

In this concluding section some of the results reviewed in the paper are summarized. In particular, analogies between classical and $\mathrm{PhC}$ waveguides are highlighted about the description of the disorder, dependence of radiation loss and backscattering on the waveguide and mode parameters and scattering regimes.

Modelling imperfections: while in classical waveguides imperfections are essentially associated with roughness at the etched sidewalls, disorder in PhCWs is much more intrinsic, because it includes for instance random variations of the radius and ellipticity of the holes, as well as random displacements of the hole position from the ideal periodic lattice. Nonetheless, in both cases, a realistic model can be developed describing the waveguide by means of only two parameters, the roughness/disorder degree $\sigma$ and the correlation length $L_{c}$, the latter measuring the distance between two uncorrelated scattering points. In classical waveguides, the roughness degree $\sigma$ strongly depends on the waveguide technology, spanning from a few $\mathrm{nm}$ in semiconductor photonic platforms to a few tens of $\mathrm{nm}$ in glass platforms (see Tab. 2); regarding the correlation length, an average value of about $50 \mathrm{~nm}$ is reported in the literature. In state-of-the art $\mathrm{PhCWs}$ fabricated on a semiconductor membrane, $\sigma$ and $L_{c}$ are typically assumed in the order of 2-3 $\mathrm{nm}$ and $40-50 \mathrm{~nm}$, respectively, but some results suggest that the scattering events generated in a disordered PhCW add coherently along the surface of the entire hole (see Sec. 6.4).

Radiation loss: In both classical waveguides and $\mathrm{PhCWs}$ disorder is responsible for power coupling between propagating mode and radiative modes (radiation losses or out-of-plane scattering). Models have been developed in both cases revealing how power radiation is proportional to the square of the disorder degree $\sigma$ (see Eq. (10) for classical waveguides and Eq.(27) for PhCWs). The group index $n_{g}$ (or equivalently the group velocity $v_{g}=c / n_{g}$ ) plays a fundamental role in the scattering processes occurring in classical and $\mathrm{PhC}$ waveguides. Radiation loss increase almost linearly at higher $n_{g}$ since the interaction time between light and disorder grows (neglecting multiple scattering in $\mathrm{PhCWs}$, see Sec. 3.3 and 6.2). However, this relation is only approximate since in principle it is possible to find two waveguides with the same group index and same parameters of the disorder but different losses, provided that they have different mode shape and hence different interaction between light and disorder. Moreover, for a given waveguide any variation in the group index is always associated with a change in the mode shape. This effect is much more pronounced in $\mathrm{PhCWs}$ than in classical waveguides, even if in the latter case the sensitivity to the disorder 
is related to the longitudinal component of the electric field and hence to the mode shape (see Sec. 3.3). For classical waveguides this results in a $n_{w}$ model depending on both group and phase effective indexes $\left(n_{g}-n_{e f f}\right)$.

Backscatter: Scaling rules similar to those valid for radiation loss apply also to backscattering. In a simplified view, backscattering depends again on the group index but now with a quadratic relation for both classical and $\mathrm{PhC}$ waveguides $\left(n_{g}^{2}\right.$, Eqs. (24) and (26)). The same consideration about the mode shape variation associated to changes of the group index $n_{g}$ are valid also for backscattering. In a more precise description, the amount of backscattered power result to be related to $\left(n_{g}-n_{e f f}\right)^{2}$ for classical waveguides $\left(n_{w}\right.$ model for backscattering), where $n_{\text {eff }}$ takes into account any variation in the mode distribution. For PhCWs the mode shape $\mathbf{e}_{k}(\mathbf{r})$ directly enters in the integral of Eq. (26). Also backscattered power has a quadratic dependence on the disorder degree $\sigma$ for both types of waveguides.

Scattering regimes: The results of a comprehensive experimental analysis carried out on different photonic platforms enable to conclude that classical waveguides affected by sidewall roughness behave as single scattering systems, independently of shape, size, and refractive index contrast of the waveguide, and independently of the light polarization state. In PhCWs the single scattering regime holds only in the dispersive (or ballistic) regime, that is as far as the group index is relatively low $\left(n_{g}<30\right)$. In this regime, the behaviour of $\mathrm{PhCWs}$ can be effectively described by the same circuit model developed for classical waveguides (see Sec. 9). At higher $n_{g}$ (diffusive regime), multiple scattering effects in $\mathrm{PhCWs}$ arise that are responsible for a number of phenomena (breakdown of the Beer-Lambert law, Fabry-Pérot-like resonances in the transmission spectrum, occurrence localized states) that make the properties of $\mathrm{PhCWs}$ strongly deviate from those of classical waveguides. Nonetheless, if the the statistical properties of PhCWs are known (see Sec. 10), a realistic circuit model for PhCWs in the diffusive regime can be developed, taking into account the above mentioned effects. It is worthwhile to note that a strict analogy can be observed between the properties of PhCWs operating in a high $n_{g}$ regime and the response of disordered CROWs at wavelengths close to the band edge.

These results enable to identify the main features of light propagation in real waveguides, to develop reliable models to evaluate the impact of waveguide imperfections on the performances of optical circuits, and ultimately to point out their limits and potentialities in practical applications.

\section{Acknowledgements}

The authors thank Marc Sorel, Michael Strain and the staff of James Watt Nanofabrication Centre (JWNC) at Glasgow University and Danxia Xu at National Research Council Canada for providing silicon photonics samples; Norbert Groete, Francisco M. Soares and Mike Wale for the fabrication of InP waveguides; Antonio Canciamilla and Stefano Grillanda for the support in the experi- 
ments. The authors gratefully acknowledge Richard De La Rue, Thomas Krauss, Philippe Lalanne and Kobus Kuipers for the fruitful discussions and suggestions. This work was partially supported by the European Community's Seventh Framework Programme FP7/2007-2013 under Grant ICT 257210 (PARADIGM) and by the Italian PRIN 2009 project Shared Access Platform to Photonic Integrated Resources (SAPPHIRE).

\section{References}

1. E. Snitzer, "Cylindrical dielectric waveguide modes," J. Opt. Soc. Am. 51, 491-498 (1961).

2. E. R. Schineller, R. P. Flam, and D. W. Wilmot, "Optical waveguides formed by proton irradiation of fused silica," J. Opt. Soc. Am. 58, 1171-1173 (1968).

3. P. K. Tien, R. Ulrich, and R. J. Martin, "Modes of propagating light waves in thin deposited semiconductor films," Applied Physics Letters 14, 291-294 (1969).

4. P. Cheben, P. J. Bock, J. H. Schmid, J. Lapointe, S. Janz, D.-X. Xu, A. Densmore, A. Delâge, B. Lamontagne, and T. J. Hall, "Refractive index engineering with subwavelength gratings for efficient microphotonic couplers and planar waveguide multiplexers," Opt. Lett. 35, 2526-2528 (2010).

5. P. J. Bock, P. Cheben, J. H. Schmid, J. Lapointe, A. Delâge, S. Janz, G. C. Aers, D.-X. $\mathrm{Xu}, \mathrm{A}$. Densmore, and T. J. Hall, "Subwavelength grating periodic structures in siliconon-insulator: a new type of microphotonic waveguide," Opt. Express 18, 20251-20262 (2010).

6. R. D. Meade, A. Devenyi, J. D. Joannopoulos, O. L. Alerhand, D. A. Smith, and K. Kash, "Novel applications of photonic band gap materials: low-loss bends and high $Q$ cavities," Journal of Applied Physics 75, 4753-4755 (1994).

7. S. G. Johnson, P. R. Villeneuve, S. Fan, and J. D. Joannopoulos, "Linear waveguides in photonic-crystal slabs," Phys. Rev. B 62, 8212-8222 (2000).

8. S. McNab, N. Moll, and Y. Vlasov, "Ultra-low loss photonic integrated circuit with membrane-type photonic crystal waveguides," Opt. Express 11, 2927-2939 (2003).

9. M. Notomi, A. Shinya, S. Mitsugi, E. Kuramochi, and H. Ryu, "Waveguides, resonators and their coupled elements in photonic crystal slabs," Opt. Express 12, 1551-1561 (2004).

10. S. G. Johnson, S. Fan, P. R. Villeneuve, J. D. Joannopoulos, and L. A. Kolodziejski, "Guided modes in photonic crystal slabs," Phys. Rev. B 60, 5751-5758 (1999).

11. A. Y. Cho, A. Yariv, and P. Yeh, "Observation of confined propagation in bragg waveguides," Applied Physics Letters 30, 471-472 (1977).

12. Y. Fink, J. N. Winn, S. Fan, C. Chen, J. Michel, J. D. Joannopoulos, and E. L. Thomas, "A dielectric omnidirectional reflector," Science 282, 1679-1682 (1998).

13. M. Duguay, Y. Kokubun, T. Koch, and L. Pfeiffer, "Antiresonant reflecting optical waveguides in sio2-si multilayer structures," Applied Physics Letters 49, 13-15 (1986).

14. D. Yin, H. Schmidt, J. Barber, and A. Hawkins, "Integrated arrow waveguides with hollow cores," Opt. Express 12, 2710-2715 (2004).

15. G. Pandraud, E. Margallo-Balbas, C.-K. Yang, and P. French, "Experimental characterization of roughness induced scattering losses in pecvd sic waveguides," Lightwave Technology, Journal of 29, 744-749 (2011).

16. J. Topolancik, F. Vollmer, R. Ilic, and M. Crescimanno, "Out-of-plane scattering from vertically asymmetric photonic crystal slab waveguides with in-plane disorder," Opt. Express 17, 12470-12480 (2009).

17. F. Ladouceur, J. Love, and T. J. Senden, "Effect of side wall roughness in buried channel waveguides," Optoelectronics, IEE Proceedings - 141, 242-248 (1994).

18. F. Ladouceur, "Roughness, inhomogeneity, and integrated optics," Lightwave Technology, Journal of 15, 1020-1025 (1997). 
19. M. Gnan, S. Thoms, D. Macintyre, R. De La Rue, and M. Sorel, "Fabrication of low-loss photonic wires in silicon-on-insulator using hydrogen silsesquioxane electron-beam resist," Electronics Letters 44, 115-116 (2008).

20. F. Morichetti, A. Canciamilla, C. Ferrari, M. Torregiani, A. Melloni, and M. Martinelli, "Roughness Induced Backscattering in Optical Silicon Waveguides," Phys. Rev. Lett. 104, 033902 (2010).

21. C. G. Poulton, C. Koos, M. Fujii, A. Pfrang, T. Schimmel, J. Leuthold, and W. Freude, "Radiation Modes and Roughness Loss in High Index-Contrast Waveguides," Selected Topics in Quantum Electronics, IEEE Journal of 12, 1306-1321 (2006).

22. J. W. Goodman, Statistical Optics (Wiley-Interscience, 2000), wiley classics library ed.

23. J. A. Ogilvy and J. R. Foster, "Rough surfaces: gaussian or exponential statistics?" Journal of Physics D: Applied Physics 22, 1243 (1989).

24. J. Lacey and F. Payne, "Radiation loss from planar waveguides with random wall imperfections," Optoelectronics, IEE Proceedings J 137, 282-288 (1990).

25. F. Payne and J. Lacey, "A theoretical analysis of scattering loss from planar optical waveguides," Optical and Quantum Electronics 26, 977-986 (1994).

26. F. Ladouceur, J. Love, and T. Senden, "Measurement of surface roughness in buried channel waveguides," Electronics Letters 28, 1321-1322 (1992).

27. M. Gottlieb, G. Brandt, and J. Conroy, "Out-of-plane scattering in optical waveguides," Circuits and Systems, IEEE Transactions on 26, 1029-1035 (1979).

28. K. K. Lee, D. R. Lim, H.-C. Luan, A. Agarwal, J. Foresi, and L. C. Kimerling, "Effect of size and roughness on light transmission in a $\mathrm{Si} / \mathrm{SiO}[\mathrm{sub} 2]$ waveguide: Experiments and model," Applied Physics Letters 77, 1617-1619 (2000).

29. S. Afifi and R. Dusséaux, "Statistical study of radiation loss from planar optical waveguides: The curvilinear coordinate method and the small perturbation method," J. Opt. Soc. Am. A 27, 1171-1184 (2010).

30. F. Grillot, L. Vivien, S. Laval, D. Pascal, and E. Cassan, "Size influence on the propagation loss induced by sidewall roughness in ultrasmall SOI waveguides," Photonics Technology Letters, IEEE 16, 1661-1663 (2004).

31. T. Barwicz and H. Haus, "Three-dimensional analysis of scattering losses due to sidewall roughness in microphotonic waveguides," Lightwave Technology, Journal of 23, 2719-2732 (2005).

32. K. K. Lee, D. R. Lim, L. C. Kimerling, J. Shin, and F. Cerrina, "Fabrication of ultralowloss $\mathrm{Si} / \mathrm{SiO} 2$ waveguides by roughness reduction," Opt. Lett. 26, 1888-1890 (2001).

33. Y. Vlasov and S. McNab, "Losses in single-mode silicon-on-insulator strip waveguides and bends," Opt. Express 12, 1622-1631 (2004).

34. Xia Fengnian, Sekaric Lidija, and Vlasov Yurii, "Ultracompact optical buffers on a silicon chip," Nat Photon 1, 65-71 (2007). 10.1038/nphoton.2006.42.

35. J. F. Bauters, M. J. R. Heck, D. John, D. Dai, M.-C. Tien, J. S. Barton, A. Leinse, R. G. Heideman, D. J. Blumenthal, and J. E. Bowers, "Ultra-low-loss high-aspect-ratio Si3N4 waveguides," Opt. Express 19, 3163-3174 (2011).

36. B. Kim, B. T. Lee, and J. G. Han, "Surface roughness of silicon oxynitride etching in $\{\mathrm{C} 2 \mathrm{~F} 6\}$ inductively coupled plasma," Solid-State Electronics 51, $366-370$ (2007).

37. W. Zhao, J. W. Bae, I. Adesida, and J. H. Jang, "Effect of mask thickness on the nanoscale sidewall roughness and optical scattering losses of deep-etched InP/InGaAsP high mesa waveguides," Journal of Vacuum Science \& Technology B: Microelectronics and Nanometer Structures 23, 2041-2045 (2005).

38. J. H. Jang, W. Zhao, J. W. Bae, D. Selvanathan, S. L. Rommel, I. Adesida, A. Lepore, M. Kwakernaak, and J. H. Abeles, "Direct measurement of nanoscale sidewall roughness of optical waveguides using an atomic force microscope," Applied Physics Letters 83, 4116-4118 (2003).

39. L. Li, T. Abe, and M. Esashi, "Smooth surface glass etching by deep reactive ion etching with SF6 and Xe gases," Journal of Vacuum Science Technology B: Microelectronics and Nanometer Structures 21, 2545-2549 (2003). 
40. D. Marcuse, "Mode Conversion Caused by Surface Imperfections of a Dielectric Slab Waveguide," Bell System Technical Journal 48, 3187-3215 (1969).

41. D. Marcuse, "Radiation Losses of Dielectric Waveguides in Terms of the Power Spectrum of the Wall Distortion Function," Bell System Technical Journal 48, 3233-3242 (1969).

42. A. Rickman, G. Reed, and F. Namavar, "Silicon-on-insulator optical rib waveguide loss and mode characteristics," Lightwave Technology, Journal of 12, 1771-1776 (1994).

43. P. Dumon, W. Bogaerts, V. Wiaux, J. Wouters, S. Beckx, J. Van Campenhout, D. Taillaert, B. Luyssaert, P. Bienstman, D. Van Thourhout, and R. Baets, "Low-loss SOI photonic wires and ring resonators fabricated with deep UV lithography," Photonics Technology Letters, IEEE 16, 1328-1330 (2004).

44. J. Cardenas, C. B. Poitras, J. T. Robinson, K. Preston, L. Chen, and M. Lipson, "Low loss etchless silicon photonic waveguides," Opt. Express 17, 4752-4757 (2009).

45. P. K. Tien, "Light Waves in Thin Films and Integrated Optics," Appl. Opt. 10, 2395-2413 (1971).

46. M. Kuznetsov and H. Haus, "Radiation loss in dielectric waveguide structures by the volume current method," Quantum Electronics, IEEE Journal of 19, 1505-1514 (1983).

47. C. Ciminelli, F. Dell'Olio, V. Passaro, and M. Armenise, "Fully three-dimensional accurate modeling of scattering loss in optical waveguides," Optical and Quantum Electronics 41, 285-298 (2009).

48. S. Johnson, M. Povinelli, M. Soljačić, A. Karalis, S. Jacobs, and J. Joannopoulos, "Roughness losses and volume-current methods in photonic-crystal waveguides," Applied Physics B 81, 283-293 (2005)

49. D. Lenz, D. Erni, and W. Bächtold, "Modal power loss coefficients for highly overmoded rectangular dielectric waveguides based on free space modes," Opt. Express 12, 1150-1156 (2004).

50. D. G. Hall, "Scattering of optical guided waves by waveguide surface roughness: a threedimensional treatment," Opt. Lett. 6, 601-603 (1981).

51. J. M. Elson, "Propagation in planar waveguides and the effects of wall roughness," Opt. Express 9, 461-475 (2001).

52. K. P. Yap, A. Delage, J. Lapointe, B. Lamontagne, J. Schmid, P. Waldron, B. Syrett, and S. Janz, "Correlation of Scattering Loss, Sidewall Roughness and Waveguide Width in Silicon-on-Insulator (SOI) Ridge Waveguides," Lightwave Technology, Journal of 27, 3999-4008 (2009).

53. E. A. J. Marcatili, "Dielectric rectangular waveguide and directional coupler for integrated optics," Bell System Technical Journal 48, 2071-2102 (1969).

54. H. Kogelnik and H. P. Weber, "Rays, stored energy, and power flow in dielectric waveguides," J. Opt. Soc. Am. 64, 174-185 (1974).

55. F. Ladouceur and L. Poladian, "Surface roughness and backscattering," Opt. Lett. 21, 1833-1835 (1996).

56. P. Verly, R. Tremblay, and J. W. Y. Lit, "Application of the effective-index method to the study of distributed feedback in corrugated waveguides: TM polarization," J. Opt. Soc. Am. 70, 1218-1221 (1980).

57. U. Glombitza and E. Brinkmeyer, "Coherent frequency-domain reflectometry for characterization of single-mode integrated-optical waveguides," IEEE Journal of Lightwave Technology 11, 1377-1384 (1993).

58. A. Canciamilla, F. Morichetti, A. Artuso, and A. Melloni, "Modelling backscattering in optical waveguides," in "18th International Workshop on Optical Waveguide Theory and Numerical Modelling," (2010).

59. D. Melati, F. Morichetti, A. Canciamilla, D. Roncelli, F. Soares, A. Bakker, and A. Melloni, "Validation of the Building-Block-Based Approach for the Design of Photonic Integrated Circuits," Lightwave Technology, Journal of 30, 3610-3616 (2012).

60. E. Kleijn, P. J. Williams, N. D. Whitbread, M. J. Wale, M. K. Smit, and X. J. Leijtens, "Sidelobes in the response of arrayed waveguide gratings caused by polarization rotation," Opt. Express 20, 22660-22668 (2012). 
61. W. Sorin and D. Baney, "Measurement of rayleigh backscattering at $1.55 \mathrm{mu}$ m with 32 mu m spatial resolution," Photonics Technology Letters, IEEE 4, 374-376 (1992).

62. F. Morichetti, A. Melloni, M. Martinelli, R. Heideman, A. Leinse, D. Geuzebroek, and A. Borreman, "Box-shaped dielectric waveguides: A new concept in integrated optics?" Lightwave Technology, Journal of 25, 2579-2589 (2007).

63. A. Melloni, R. Costa, G. Cusmai, and F. Morichetti, "The role of index contrast in dielectric optical waveguides," International Journal of Materials and Product Technology 34 (2009).

64. M. Skorobogatiy, G. Bégin, and A. Talneau, "Statistical analysis of geometrical imperfections from the images of 2D photonic crystals," Opt. Express 13, 2487-2502 (2005).

65. M. Patterson and S. Hughes, Optical Properties of Photonic Structures: Interplay of Order and Disorder (CRC Press, Boca Raton, FL 33487-2742, 2012), chap. The Disorder Problem for Slow-Light Photonic Crystal Waveguides.

66. W. Bogaerts, P. Bienstman, and R. Baets, "Scattering at sidewall roughness in photonic crystal slabs," Opt. Lett. 28, 689-691 (2003).

67. M. L. Povinelli, S. G. Johnson, E. Lidorikis, J. D. Joannopoulos, and M. Soljačić, "Effect of a photonic band gap on scattering from waveguide disorder," Applied Physics Letters 84, 3639-3641 (2004).

68. D. Gerace and L. C. Andreani, "Disorder-induced losses in photonic crystal waveguides with line defects," Opt. Lett. 29, 1897-1899 (2004).

69. L. C. Andreani and D. Gerace, "Light matter interaction in photonic crystal slabs," physica status solidi (b) 244, 3528-3539 (2007).

70. G. Lecamp, J. P. Hugonin, and P. Lalanne, "Theoretical and computational concepts for periodic optical waveguides," Opt. Express 15, 11042-11060 (2007).

71. S. Mazoyer, J. P. Hugonin, and P. Lalanne, "Disorder-Induced Multiple Scattering in Photonic-Crystal Waveguides," Phys. Rev. Lett. 103, 063903 (2009).

72. V. Savona, "Electromagnetic modes of a disordered photonic crystal," Phys. Rev. B 83, 085301 (2011).

73. V. Savona, "Erratum: Electromagnetic modes of a disordered photonic crystal [Phys. Rev. B 83, 085301 (2011)]," Phys. Rev. B 86, 079907 (2012).

74. M. Minkov and V. Savona, "Effect of hole-shape irregularities on photonic crystal waveguides," Opt. Lett. 37, 3108-3110 (2012).

75. T. N. Langtry, A. A. Asatryan, L. C. Botten, C. M. de Sterke, R. C. McPhedran, and P. A. Robinson, "Effects of disorder in two-dimensional photonic crystal waveguides," Phys. Rev. E 68, 026611 (2003).

76. K.-C. Kwan, X. Zhang, Z.-Q. Zhang, and C. T. Chan, "Effects due to disorder on photonic crystal-based waveguides," Applied Physics Letters 82, 4414-4416 (2003).

77. S. Fan, P. R. Villeneuve, and J. D. Joannopoulos, "Theoretical investigation of fabricationrelated disorder on the properties of photonic crystals," Journal of Applied Physics 78, 1415-1418 (1995).

78. S. Hughes, L. Ramunno, J. F. Young, and J. E. Sipe, "Extrinsic Optical Scattering Loss in Photonic Crystal Waveguides: Role of Fabrication Disorder and Photon Group Velocity," Phys. Rev. Lett. 94, 033903 (2005).

79. E. Kuramochi, M. Notomi, S. Hughes, A. Shinya, T. Watanabe, and L. Ramunno, "Disorder-induced scattering loss of line-defect waveguides in photonic crystal slabs," Phys. Rev. B 72, 161318 (2005).

80. M. Patterson, S. Hughes, S. Schulz, D. M. Beggs, T. P. White, L. O'Faolain, and T. F. Krauss, "Disorder-induced incoherent scattering losses in photonic crystal waveguides: Bloch mode reshaping, multiple scattering, and breakdown of the Beer-Lambert law," Phys. Rev. B 80, 195305 (2009).

81. M. Patterson and S. Hughes, "Theory of disorder-induced coherent scattering and light localization in slow-light photonic crystal waveguides," Journal of Optics 12, 104013 (2010).

82. L. O'Faolain, S. A. Schulz, D. M. Beggs, T. P. White, M. Spasenović, L. Kuipers, F. Morichetti, A. Melloni, S. Mazoyer, J. P. Hugonin, P. Lalanne, and T. F. Krauss, 
"Loss engineered slow light waveguides," Opt. Express 18, 27627-27638 (2010).

83. S. McNab, N. Moll, and Y. Vlasov, "Ultra-low loss photonic integrated circuit with membrane-type photonic crystal waveguides," Opt. Express 11, 2927-2939 (2003).

84. J. Li, T. P. White, L. O'Faolain, A. Gomez-Iglesias, and T. F. Krauss, "Systematic design of flat band slow light in photonic crystal waveguides," Opt. Express 16, 6227-6232 (2008).

85. L. H. Frandsen, A. V. Lavrinenko, J. Fage-Pedersen, and P. I. Borel, "Photonic crystal waveguides with semi-slow light and tailored dispersion properties," Opt. Express 14, 9444-9450 (2006).

86. A. Y. Petrov and M. Eich, "Zero dispersion at small group velocities in photonic crystal waveguides," Applied Physics Letters 85, 4866-4868 (2004).

87. D. Mori and T. Baba, "Dispersion-controlled optical group delay device by chirped photonic crystal waveguides," Applied Physics Letters 85, 1101-1103 (2004).

88. L. O'Faolain, X. Yuan, D. McIntyre, S. Thoms, H. Chong, R. De La Rue, and T. Krauss, "Low-loss propagation in photonic crystal waveguides," Photonics Technology Letters, IEEE 25, 1454-1455 (2006).

89. M. Notomi, T. Tanabe, A. Shinya, E. Kuramochi, H. Taniyama, S. Mitsugi, and M. Morita, "Nonlinear and adiabatic control of high-Qphotonic crystal nanocavities," Opt. Express 15, 17458-17481 (2007).

90. F. Morichetti, A. Canciamilla, M. Martinelli, A. Samarelli, R. M. D. L. Rue, M. Sorel, and A. Melloni, "Coherent backscattering in optical microring resonators," Applied Physics Letters 96, 081112 (2010).

91. A. Gomez-Iglesias, D. O'Brien, L. O'Faolain, A. Miller, and T. F. Krauss, "Direct measurement of the group index of photonic crystal waveguides via Fourier transform spectral interferometry," Applied Physics Letters 90, 261107 (2007).

92. A. Parini, P. Hamel, A. D. Rossi, S. Combrié, N.-V.-Q. Tran, Y. Gottesman, R. Gabet, A. Talneau, Y. Jaouën, and G. Vadalà, "Time-Wavelength Reflectance Maps of Photonic Crystal Waveguides: A New View on Disorder-Induced Scattering," J. Lightwave Technol. 26, 3794-3802 (2008).

93. C. Canavesi, F. Morichetti, A. Canciamilla, F. Persia, and A. Melloni, "Polarization- and Phase-Sensitive Low-Coherence Interferometry Setup for the Characterization of Integrated Optical Components," Lightwave Technology, Journal of 27, 3062-3074 (2009).

94. R. J. P. Engelen, D. Mori, T. Baba, and L. Kuipers, "Two Regimes of Slow-Light Losses Revealed by Adiabatic Reduction of Group Velocity," Phys. Rev. Lett. 101, 103901 (2008).

95. L. O'Faolain, T. P. White, D. O'Brien, X. Yuan, M. D. Settle, and T. F. Krauss, "Dependence of extrinsic loss on group velocity in photonic crystal waveguides," Opt. Express 15, 13129-13138 (2007).

96. Y. A. Vlasov and S. J. McNab, "Coupling into the slow light mode in slab-type photonic crystal waveguides," Opt. Lett. 31, 50-52 (2006).

97. S. G. Johnson, P. Bienstman, M. A. Skorobogatiy, M. Ibanescu, E. Lidorikis, and J. D. Joannopoulos, "Adiabatic theorem and continuous coupled-mode theory for efficient taper transitions in photonic crystals," Phys. Rev. E 66, 066608 (2002).

98. P. Pottier, M. Gnan, and R. M. D. L. Rue, "Efficient coupling into slow-light photonic crystal channel guides using photonic crystal tapers," Opt. Express 15, 6569-6575 (2007).

99. J. P. Hugonin, P. Lalanne, T. P. White, and T. F. Krauss, "Coupling into slow-mode photonic crystal waveguides," Opt. Lett. 32, 2638-2640 (2007).

100. S. A. Schulz, L. O'Faolain, D. M. Beggs, T. P. White, A. Melloni, and T. F. Krauss, "Dispersion engineered slow light in photonic crystals: a comparison," Journal of Optics 12, 104004 (2010).

101. A. Hosseini, X. Xu, D. N. Kwong, H. Subbaraman, W. Jiang, and R. T. Chen, "On the role of evanescent modes and group index tapering in slow light photonic crystal waveguide coupling efficiency," Applied Physics Letters 98, 031107-031107-3 (2011).

102. J. Li, L. O'Faolain, S. Schulz, and T. Krauss, "Low loss propagation in slow light photonic crystal waveguides at group indices up to 60," Photonics and nanostructuresFundamentals and applications 10, 589-593 (2012). 
103. M. Patterson, S. Hughes, S. Combrié, N.-V.-Q. Tran, A. De Rossi, R. Gabet, and Y. Jaouën, "Disorder-Induced Coherent Scattering in Slow-Light Photonic Crystal Waveguides," Phys. Rev. Lett. 102, 253903 (2009).

104. A. Petrov, M. Krause, and M. Eich, "Backscattering and disorder limits in slow light photonic crystal waveguides," Opt. Express 17, 8676-8684 (2009).

105. F. Wang, J. S. Jensen, J. Mørk, and O. Sigmund, "Systematic design of loss-engineered slow-light waveguides," J. Opt. Soc. Am. A 29, 2657-2666 (2012).

106. B. Wang, S. Mazoyer, J. P. Hugonin, and P. Lalanne, "Backscattering in monomode periodic waveguides," Phys. Rev. B 78, 245108 (2008).

107. W. Song, R. A. Integlia, and W. Jiang, "Slow light loss due to roughness in photonic crystal waveguides: An analytic approach," Phys. Rev. B 82, 235306 (2010).

108. A. Ishimaru, Wave Propagation and Scattering in Random Media, IEEE/OUP series on electromagnetic wave theory (Academic Press, 1978).

109. P. Sheng, Introduction to Wave Scattering, Localization, and Mesoscopic Phenomena (Academic Press, 1995).

110. N. Le Thomas, H. Zhang, J. Jágerská, V. Zabelin, R. Houdré, I. Sagnes, and A. Talneau, "Light transport regimes in slow light photonic crystal waveguides," Phys. Rev. B 80, 125332 (2009).

111. L. Ryzhik, G. Papanicolaou, and J. B. Keller, "Transport equations for elastic and other waves in random media," Wave Motion 24, 327-370 (1996).

112. M. P. V. Albada and A. Lagendijk, "Observation of Weak Localization of Light in a Random Medium," Phys. Rev. Lett. 55, 2692-2695 (1985).

113. P.-E. Wolf and G. Maret, "Weak Localization and Coherent Backscattering of Photons in Disordered Media," Phys. Rev. Lett. 55, 2696-2699 (1985).

114. M. C. W. van Rossum and T. M. Nieuwenhuizen, "Multiple scattering of classical waves: microscopy, mesoscopy, and diffusion," Rev. Mod. Phys. 71, 313-371 (1999).

115. B. A. van Tiggelen, A. Lagendijk, M. P. van Albada, and A. Tip, "Speed of light in random media," Phys. Rev. B 45, 12233-12243 (1992).

116. S. Mazoyer, A. Baron, J.-P. Hugonin, P. Lalanne, and A. Melloni, "Slow pulses in disordered photonic-crystal waveguides," Appl. Opt. 50, G113-G117 (2011).

117. A. Baron, S. Mazoyer, W. Smigaj, and P. Lalanne, "Attenuation Coefficient of SingleMode Periodic Waveguides," Phys. Rev. Lett. 107, 153901 (2011).

118. J. Topolancik, B. Ilic, and F. Vollmer, "Experimental Observation of Strong Photon Localization in Disordered Photonic Crystal Waveguides," Phys. Rev. Lett. 99, 253901 (2007).

119. S. Combrié, N.-V.-Q. Tran, E. Weidner, A. D. Rossi, S. Cassette, P. Hamel, Y. Jaouën, R. Gabet, and A. Talneau, "Investigation of group delay, loss, and disorder in a photonic crystal waveguide by low-coherence reflectometry," Applied Physics Letters 90, 231104 (2007).

120. M. Sandtke, R. J. P. Engelen, H. Schoenmaker, I. Attema, H. Dekker, I. Cerjak, J. P. Korterik, F. B. Segerink, and L. Kuipers, "Novel instrument for surface plasmon polariton tracking in space and time," Review of Scientific Instruments 79, 013704 (2008).

121. T. Baba, D. Mori, K. Inoshita, and Y. Kuroki, "Light localizations in photonic crystal line defect waveguides," Selected Topics in Quantum Electronics, IEEE Journal of 10, 484-491 (2004).

122. D. Mori and T. Baba, "Wideband and low dispersion slow light by chirped photonic crystal coupled waveguide," Opt. Express 13, 9398-9408 (2005).

123. M. Soljačić and J. D. Joannopoulos, "Enhancement of nonlinear effects using photonic crystals," Nat. Mater. 3, 211-219 (2004). 10.1038/nmat1097.

124. M. Schneider and S. Mookherjea, "Modeling Transmission Time of Silicon Nanophotonic Waveguides," Photonics Technology Letters, IEEE 24, 1418-1420 (2012).

125. P. Healey, "Statistics of Rayleigh Backscatter From a Single-Mode Fiber," Communications, IEEE Transactions on 35, 210-214 (1987).

126. P. Gysel and R. Staubli, "Statistical properties of Rayleigh backscattering in single-mode fibers," Lightwave Technology, Journal of 8, 561-567 (1990). 
127. F. Morichetti, A. Canciamilla, and A. Melloni, "Statistics of backscattering in optical waveguides," Opt. Lett. 35, 1777-1779 (2010).

128. J. W. Goodman, Statistical Optics (Wiley-Interscience, 1985).

129. W. Yun-ping and Z. Dian-lin, "Reshaping, path uncertainty, and superluminal traveling," Phys. Rev. A 52, 2597-2600 (1995).

130. Aspic by Filarete, www.aspicdesign.com.

131. S. Mazoyer, P. Lalanne, J. Rodier, J. Hugonin, M. Spasenović, L. Kuipers, D. Beggs, and T. Krauss, "Statistical fluctuations of transmission in slow light photonic-crystal waveguides," Opt. Express 18, 14654-14663 (2010).

132. P. Pradhan and N. Kumar, "Localization of light in coherently amplifying random media," Phys. Rev. B 50, 9644-9647 (1994).

133. Y. A. Vlasov, M. A. Kaliteevski, and V. V. Nikolaev, "Different regimes of light localization in a disordered photonic crystal," Phys. Rev. B 60, 1555-1562 (1999).

134. P. D. García, S. Smolka, S. Stobbe, and P. Lodahl, "Density of states controls Anderson localization in disordered photonic crystal waveguides," Phys. Rev. B 82, 165103 (2010).

135. S. Smolka, H. Thyrrestrup, L. Sapienza, T. B. Lehmann, K. R. Rix, L. S. Froufe-Pérez, P. D. García, and P. Lodahl, "Probing the statistical properties of Anderson localization with quantum emitters," New Journal of Physics 13, 063044 (2011).

136. M. Spasenović, D. M. Beggs, P. Lalanne, T. F. Krauss, and L. Kuipers, "Measuring the spatial extent of individual localized photonic states," Phys. Rev. B 86, 155153 (2012).

137. M. Borselli, K. Srinivasan, P. E. Barclay, and O. Painter, "Rayleigh scattering, mode coupling, and optical loss in silicon microdisks," Applied Physics Letters 85, 3693-3695 (2004).

138. M. Borselli, T. Johnson, and O. Painter, "Beyond the Rayleigh scattering limit in high-Q silicon microdisks: theory and experiment," Opt. Express 13, 1515-1530 (2005).

139. Schliesser A., Riviere R., Anetsberger G., Arcizet O., and Kippenberg T. J., "Resolvedsideband cooling of a micromechanical oscillator," Nat Phys 4, 415-419 (2008). 10.1038/nphys939.

140. G. Anetsberger, R. Riviere, A. Schliesser, O. Arcizet, and J. T. Kippenberg, "Ultralowdissipation optomechanical resonators on a chip," Nat Photon 2, 627-633 (2008). 10.1038/nphoton.2008.199

141. J. Kalkman, A. Polman, T. Kippenberg, K. Vahala, and M. L. Brongersma, "Erbiumimplanted silica microsphere laser," Nuclear Instruments and Methods in Physics Research Section B: Beam Interactions with Materials and Atoms 242, 182-185 (2006).

142. T. J. Kippenberg, S. M. Spillane, and K. J. Vahala, "Modal coupling in traveling-wave resonators," Opt. Lett. 27, 1669-1671 (2002).

143. W. Bogaerts, R. Baets, P. Dumon, V. Wiaux, S. Beckx, D. Taillaert, B. Luyssaert, J. Van Campenhout, P. Bienstman, and D. Van Thourhout, "Nanophotonic waveguides in siliconon-insulator fabricated with CMOS technology," Lightwave Technology, Journal of 23, 401-412 (2005).

144. B. E. Little, J.-P. Laine, and S. T. Chu, "Surface-roughness-induced contradirectional coupling in ring and disk resonators," Opt. Lett. 22, 4-6 (1997).

145. B. E. Little and S. T. Chu, "Estimating surface-roughness loss and output coupling in microdisk resonators," Opt. Lett. 21, 1390-1392 (1996).

146. M. L. Gorodetsky, A. D. Pryamikov, and V. S. Ilchenko, "Rayleigh scattering in high-Q microspheres," J. Opt. Soc. Am. B 17, 1051-1057 (2000).

147. J. C̆tyroký, I. Richter, and M. Šn̆or, "Dual resonance in a waveguide-coupled ring microresonator," Optical and Quantum Electronics 38, 781-797 (2006).

148. G. C. Ballesteros, J. Matres, J. Martí, and C. J. Oton, "Characterizing and modeling backscattering in silicon microring resonators," Opt. Express 19, 24980-24985 (2011).

149. A. Melloni, A. Canciamilla, C. Ferrari, F. Morichetti, L. O'Faolain, T. Krauss, R. D. L. Rue, A. Samarelli, and M. Sorel, "Tunable delay lines in silicon photonics: Coupled resonators and photonic crystals, a comparison," Photonics Journal, IEEE 2, 181-194 (2010).

150. A. Simard, N. Ayotte, Y. Painchaud, S. Bedard, and S. LaRochelle, "Impact of Sidewall 
Roughness on Integrated Bragg Gratings," Lightwave Technology, Journal of 29, 36933704 (2011).

151. A. Yariv, Y. Xu, R. K. Lee, and A. Scherer, "Coupled-resonator optical waveguide: a proposal and analysis," Opt. Lett. 24, 711-713 (1999).

152. F. Morichetti, C. Ferrari, A. Canciamilla, and A. Melloni, "The first decade of coupled resonator optical waveguides: bringing slow light to applications," Laser \& Photonics Reviews 6, 74-96 (2012).

153. J. Goeckeritz and S. Blair, "Optical characterization of coupled resonator slow-light rib waveguides," Opt. Express 18, 18190-18199 (2010).

154. F. Xia, M. Rooks, L. Sekaric, and Y. Vlasov, "Ultra-compact high order ring resonator filters using submicron silicon photonic wires for on-chip optical interconnects," Opt. Express 15, 11934-11941 (2007).

155. M. Hossein-Zadeh and K. J. Vahala, "An optomechanical oscillator on a silicon chip," Selected Topics in Quantum Electronics, IEEE Journal of 16, 276 -287 (2010).

156. A. M. Kapitonov and V. N. Astratov, "Observation of nanojet-induced modes with small propagation losses in chains of coupled spherical cavities," Opt. Lett. 32, 409-411 (2007).

157. S. Mookherjea, J. S. Park, S. Yang, and P. R. Bandaru, "Localization in silicon nanophotonic slow-light waveguides," Nat Photon 2, 90-93 (2008).

158. T. Karle, D. Brown, R. Wilson, M. Steer, and T. Krauss, "Planar photonic crystal coupled cavity waveguides," Selected Topics in Quantum Electronics, IEEE Journal of 8, 909 918 (2002).

159. A. Melloni, F. Morichetti, and M. Martinelli, "Linear and nonlinear pulse propagation in coupled resonator slow-wave optical structures," Optical and Quantum Electronics 35, 365-379 (2003).

160. A. Melloni and M. Martinelli, "Synthesis of Direct-Coupled-Resonators bandpass filters for WDM systems," Journal of Lightwave Technology 20, 296 (2002).

161. S. Mookherjea and A. Oh, "Effect of disorder on slow light velocity in optical slow-wave structures," Optics Letters 32, 289-291 (2007).

162. C. Ferrari, F. Morichetti, and A. Melloni, "Disorder in coupled-resonator optical waveguides," Journal of the Optical Society of America B 26, 858-866 (2009).

163. H.-Y. Ryu, J.-K. Hwang, and Y.-H. Lee, "Effect of size nonuniformities on the band gap of two-dimensional photonic crystals," Phys. Rev. B 59, 5463-5469 (1999).

164. A. H. Firester, M. E. Heller, and P. Sheng, "Knife-edge scanning measurements of subwavelength focused light beams," Appl. Opt. 16, 1971-1974 (1977).

165. M. L. Cooper, G. Gupta, M. A. Schneider, W. M. J. Green, S. Assefa, F. Xia, Y. A. Vlasov, and S. Mookherjea, "Statistics of light transport in 235-ring silicon coupled-resonator optical waveguides," Opt. Express 18, 26505-26516 (2010).

166. A. Canciamilla, M. Torregiani, C. Ferrari, F. Morichetti, R. M. De La Rue, A. Samarelli, M. Sorel, and A. Melloni, "Silicon coupled-ring resonator structures for slow light applications: potential, impairments and ultimate limits," Journal of Optics 12, 104008 (2010).

167. S. T. Chu, W. Pan, S. Sato, T. Kaneko, B. E. Little, and Y. Kokubun, "Wavelength trimming of a microring resonator filter by means of a UV sensitive polymer overlay," Photonics Technology Letters, IEEE 11, 688-690 (1999).

168. D. K. Sparacin, C. yin Hong, L. C. Kimerling, J. Michel, J. P. Lock, and K. K. Gleason, "Trimming of microring resonators by photo-oxidation of a plasma-polymerized organosilane cladding material," Opt. Lett. 30, 2251-2253 (2005).

169. J. Schrauwen, D. V. Thourhout, and R. Baets, "Trimming of silicon ring resonator by electron beam induced compaction and strain," Opt. Express 16, 3738-3743 (2008).

170. A. Canciamilla, S. Grillanda, F. Morichetti, C. Ferrari, J. Hu, J. D. Musgraves, K. Richardson, A. Agarwal, L. C. Kimerling, and A. Melloni, "Photo-induced trimming of coupled ring-resonator filters and delay lines in as2s3 chalcogenide glass," Opt. Lett. 36, 4002-4004 (2011).

171. Y. Shen, I. B. Divliansky, D. N. Basov, and S. Mookherjea, "Electric-field-driven nanooxidation trimming of silicon microrings and interferometers," Opt. Lett. 36, 2668-2670 
(2011).

172. A. Canciamilla, F. Morichetti, S. Grillanda, P. Velha, M. Sorel, V. Singh, A. Agarwal, L. C. Kimerling, and A. Melloni, "Photo-induced trimming of chalcogenide-assisted silicon waveguides," Opt. Express 20, 15807-15817 (2012).

173. S. Prorok, A. Y. Petrov, M. Eich, J. Luo, and A. K.-Y. Jen, "Trimming of high-q-factor silicon ring resonators by electron beam bleaching," Opt. Lett. 37, 3114-3116 (2012).

174. A. H. Atabaki, A. A. Eftekhar, M. Askari, and A. Adibi, "Accurate post-fabrication trimming of ultra-compact resonators on silicon," Opt. Express 21, 14139-14145 (2013). 